\title{
A PFAFFIAN REPRESENTATION FOR FLAT ASEP
}

\author{
JANOSCH ORTMANN, JEREMY QUASTEL, AND DANIEL REMENIK
}

\begin{abstract}
We obtain a Fredholm Pfaffian formula for an appropriate generating function of the height function of the asymmetric simple exclusion process starting from flat (periodic) initial data. Formal asymptotics lead to the GOE Tracy-Widom distribution.
\end{abstract}

\section{CONTENTS}

1. Introduction 1

2. Main results 3

2.1. Preliminaries 3

2.2. Fredholm Pfaffian formula 5

2.3. Moment formula and emergence of the Pfaffian structure 7

3. Moment formula: contour deformation 11

3.1. Poles of the integrand 11

3.2. Contour deformation and computation of the limit 13

3.3. Simplification of the $\mathfrak{h}$ factors 17

4. Moment formula: Pfaffian structure 19

4.1. Case $k_{\mathrm{u}}$ even 19

4.2. Extension to general $k_{\mathrm{u}}$

5. Generating function 30

6. Moment formulas for flat KPZ/stochastic heat equation $\quad 35$

Appendix A. Formal GOE asymptotics for flat ASEP 38

A.1. Mellin-Barnes representation 38

A.2. Computation of the limit 43

A.3. Fredholm Pfaffian formula for GOE 46

Appendix B. Fredholm Pfaffians $\quad 50$

B.1. Pfaffians 50

B.2. Fredholm determinants 53

B.3. Fredholm Pfaffians on $L^{2}$ spaces $\quad 54$

References $\quad 57$

\section{INTRODUCTION}

In this article, we consider the one-dimensional asymmetric simple exclusion process (ASEP) with flat initial data, meaning that initially even sites are occupied and odd sites are empty. The particles then perform nearest neighbour asymmetric random walks, in continuous time, the only interaction being that jumps to already occupied sites are suppressed. ASEP is perhaps the most popular of the small class of special partially solvable discretizations of the Kardar-Parisi-Zhang (KPZ) equation. In recent years, a series of 
breakthroughs on these models has led to exact formulas for one point distributions of the solutions of KPZ for very special initial data. The first formulas were for the narrow wedge and half-Brownian initial conditions [ACQ11; CQ13], which were obtained by taking limits of earlier formulas for ASEP with step and step-Bernoulli initial conditions due to Tracy and Widom [TW09a; TW09b]. The remaining key initial data, flat, half-flat and Brownian, are more challenging and had to wait several years ([BCFV14; OQR14], and the present work). Meanwhile there was a parallel effort in the physics community working directly from KPZ, using the method of replicas to derive rigorous formulas for the moments, and then writing divergent series for appropriate generating functions for the one point distributions of KPZ, which could then be formally manipulated into convergent Fredholm series [CDR10; Dot10; IS11; IS13].

In this context, P. Le Doussal and P. Calabrese [LDC12] derived what in mathematics would be called conjectural expressions for the flat case, as Fredholm Pfaffians. To a certain extent, this work is an attempt to make sense of their remarkable, but highly non-rigorous computations. Our main results provide analogous formulas for flat ASEP. Formal asymptotics give the correct fluctuations in the large time limit, which in this case correspond to the GOE Tracy-Widom distribution [TW96], in a new form. In the weakly asymmetric limit one obtains versions of the formulas in [LDC12]. However the technicalities of this, as well as those of providing a rigorous justification of the large time asymptotics, are so involved that we have left them for a future article.

It should be noted that an earlier formula existed for the one point distribution of ASEP with flat initial data, due to E. Lee [Lee10]. However, this formula is similar to the first formulas of Tracy and Widom for the step case Tracy and Widom [TW08b] and are not in a suitable form for asymptotics, even at a formal level. In the step case, it already required an extraordinary argument [TW08a] to rewrite this as what is now referred to as a small contour formula, which is indeed suitable for asymptotics [TW09a]. In the flat case this method has not been successful so far, and thus we follow an alternative approach.

In a prequel article [OQR14], we derived a formula for a generating function in the half-flat case, meaning that initially only even positive sites are occupied. Because such data is left-finite, meaning that there is a leftmost particle, the duality methods of [BCS14] apply, and with suitable guessing, inspired, in part, by the formulas of [Lee10], one is able to obtain an exact solution to the duality equations, which provide formulas for the exponential moments of the ASEP height function and can then be turned into a certain generating function.

In this article we take a limit of the half-flat moment formula probing into the positive region, which reproduces the flat initial data. We follow the broad lines of [LDC12]. The asymptotics is extremely interesting, nonstandard, and unusually involved. One is forced to make a large deformation of contours, and the flat formula is an enormous sum of the residues passed as one makes this deformation. In this sum, a pairing structure arises which leads to the emergence of Pfaffians.

Naturally, the remarkable formulas beg the question whether there is a more direct approach, and some recent physics work have indicated possibilities in this direction [DNWBC14; CD14].

Outline. The rest of the article is organized as follows. Our main results are discussed in Section 2. Section 3 contains the derivation of the flat ASEP moment formulas as a limit of our earlier half-flat formulas. Section 4 is devoted to unravelling the Pfaffian structure behind the moment formulas and rewriting them in a simpler way. The resulting formulas are then summed in Section 5 to form a generating function and showing how, in a certain case, they lead to a Fredholm Pfaffian. Section 6 contains a discussion of analogous results for the moments of the solution of the KPZ/SHE equation (or, more precisely, the delta Bose gas) with flat initial data. Appendix A presents a formal critical 
point analysis supporting the conjecture that the ASEP height function fluctuations are described in the long time limit by the GOE Tracy-Widom distribution when the initial data is flat. As explained in Remark 2.2.vii, the rigorous asymptotics cannot be obtained directly by these methods and remains the subject for future work. Finally, Appendix B contains an overview of Pfaffians and Fredholm Pfaffians.

\section{MAin Results}

2.1. Preliminaries. The one-dimensional asymmetric simple exclusion process (ASEP) with jump rates $p \in[0,1 / 2)$ to the right and $q=1-p \geq p$ to the left is the continuous time Markov process with state space $\{0,1\}^{\mathbb{Z}}$ and generator

$$
\begin{aligned}
\mathcal{L} f(\eta)= & \sum_{x \in \mathbb{Z}} p \eta(x)(1-\eta(x+1))\left(f\left(\eta-\mathbf{1}_{x}+\mathbf{1}_{x+1}\right)-f(\eta)\right) \\
& +q \eta(x+1)(1-\eta(x))\left(f\left(\eta+\mathbf{1}_{x}-\mathbf{1}_{x+1}\right)-f(\eta)\right),
\end{aligned}
$$

where $\mathbf{1}_{x}(y)=1$ if $y=x$ and 0 otherwise. Writing $\hat{\eta}(x)=2 \eta(x)-1$, we define the ASEP height function by

$$
h(t, x)= \begin{cases}2 N_{0}^{\text {flux }}(t)+\sum_{0<y \leq x} \hat{\eta}(t, y), & x>0 \\ 2 N_{0}^{\text {flux }}(t), & x=0 \\ 2 N_{0}^{\text {flux }}(t)-\sum_{x<y \leq 0} \hat{\eta}(t, y), & x<0\end{cases}
$$

where $N_{0}^{\text {flux }}(t)$ is the net number of particles which crossed from site 1 to 0 up to time $t$, meaning that particle jumps $1 \rightarrow 0$ are counted as +1 and jumps $0 \rightarrow 1$ are counted as -1 . For $x \notin \mathbb{Z}$ we define $h(t, x)$ by linear interpolation. With these conventions $h(t, x)$ is made of line segments with slopes \pm 1 , and it evolves with its own Markovian dynamics, which flips $\vee \mapsto \wedge$ at rate $q$ and $\wedge \mapsto \vee$ at rate $p$. For $q>p$, the general trend is an upwards moving height function.

An important parameter in the formulas will be

$$
\tau=p / q \in[0,1] .
$$

We are primarily concerned with the flat,

$$
\eta_{0}^{\text {flat }}(x)=\mathbf{1}_{x \in 2 \mathbb{Z}}
$$

and half-flat,

$$
\eta_{0}^{\mathrm{h}-\mathrm{fl}}(x)=\mathbf{1}_{x \in 2 \mathbb{Z}_{>0}}
$$

initial conditions (with the notation $\mathbb{Z}_{>n}=\{n+1, n+2, \ldots\}$ which will be used throughout). The superscripts flat and h-fl will be used for probabilities and expectations computed with respect to these initial conditions.

In order to state our results we need to recall some definitions. Given a measure space $(X, \Sigma, \mu)$, the Fredholm Pfaffian (introduced in [Rai00]) of an anti-symmetric $2 \times 2$-matrix kernel $K\left(\lambda_{1}, \lambda_{2}\right)=\left[\begin{array}{ccc}K_{1,1}\left(\lambda_{1}, \lambda_{2}\right) & K_{1,2}\left(\lambda_{1}, \lambda_{2}\right) \\ -K_{1,2}\left(\lambda_{2}, \lambda_{1}\right) & K_{2,2}\left(\lambda_{1}, \lambda_{2}\right)\end{array}\right]$ acting on $L^{2}(X)$ is defined as the (formal) series

$$
\operatorname{Pf}[J-K]_{L^{2}(X)}=\sum_{k \geq 0} \frac{(-1)^{k}}{k !} \int_{X^{k}} \mu\left(d \lambda_{1}\right) \cdots \mu\left(d \lambda_{k}\right) \operatorname{Pf}\left[K\left(\lambda_{a}, \lambda_{b}\right)\right]_{a, b=1}^{k},
$$

where, we recall, the Pfaffian of a $2 k \times 2 k$ matrix $A=\left\{a_{i, j}\right\}$ is given by

$$
\operatorname{Pf}[A]=\frac{1}{2^{k} k !} \sum_{\sigma \in S_{2 n}} \operatorname{sgn}(\sigma) \prod_{i=1}^{k} a_{\sigma(2 i-1), \sigma(2 i)} .
$$

Appendix B surveys basic facts about Fredholm Pfaffians. 
We will also need various $q$-analogues of standard calculus functions. The $q$ here is the $q$ in quantum calculus [GR04]. Our parameter (2.2) came with the unfortuitous name $\tau$ instead of $q$, since historically the left jump rate was called $q$. In an attempt to keep with standard practice, we will use $q$ when discussing the definitions of the $q$-deformed functions, but set $q \mapsto \tau$ within the computations. The standard $q$-numbers are given by

$$
[k]_{q}=\frac{1-q^{k}}{1-q} .
$$

Based on them one defines the q-factorial

$$
n_{q} !=[1]_{q}[2]_{q} \ldots[n]_{q}=\frac{(q ; q)_{n}}{(1-q)^{n}},
$$

where the $q$-Pochhammer symbols are given by

$$
(a ; q)_{n}=\prod_{k=0}^{n-1}\left(1-q^{k} a\right) .
$$

This last definition can be extended directly to $n=\infty$ when $|q|<1$, yielding the infinite $q$-Pochhammer symbols. The standard $q$-exponentials are then defined as

$$
e_{q}(z)=\frac{1}{((1-q) z ; q)_{\infty}} \quad \text { and } \quad E_{q}(z)=(-(1-q) z ; q)_{\infty}
$$

or (thanks to the $q$-Binomial Theorem) through their series representations

$$
e_{q}(z)=\sum_{k \geq 0} \frac{z^{k}}{k_{q} !} \quad \text { and } \quad E_{q}(z)=\sum_{k \geq 0} \frac{q^{\frac{1}{2} k(k-1)} z^{k}}{k_{q} !} .
$$

The first series converges absolutely only for $|z|<1$, but it extends analytically to $\Re(z)<0$ through the formula in (2.4), where it approximates $\exp (z)$ well as $q \nearrow 1$. And, of course, $E_{q}(z)=e_{q}(-z)$. In [OQR14] we obtained a formula for $\mathbb{E}^{\mathrm{h}-\mathrm{fl}}\left[e_{\tau}\left(\zeta \tau^{h(t, x) / 2}\right)\right]$.

In the flat case one is naturally led to use a different kind of $q$-exponential. The symmetric q-numbers [NG94; FT95] are given by

$$
\widetilde{[k]_{q}}=\frac{q^{k / 2}-q^{-k / 2}}{q^{1 / 2}-q^{-1 / 2}}
$$

and the symmetric q-factorial by

$$
\widetilde{k_{q} !}=\widetilde{[1]_{q}} \widetilde{[2]_{q}} \ldots \widetilde{[k]_{q}}=q^{-\frac{1}{4} k(k-1)} k_{q} !
$$

with $\widetilde{0_{q} !}=1$. The symmetric $q$-exponential function is then defined as

$$
\exp _{q}(z)=\sum_{k=0}^{\infty} \frac{z^{k}}{\widehat{k_{q} !}}=\sum_{k=0}^{\infty} \frac{q^{\frac{1}{4} k(k-1)}}{k_{q} !} z^{k} .
$$

The function $f(z)=\exp _{q}(z)$ is the unique solution of

$$
\frac{\delta_{q} f(z)}{\delta_{q} z}=f(z), \quad f(0)=1
$$

where the symmetric $q$-difference operator is defined by $\delta_{q} f(z)=f\left(q^{1 / 2} z\right)-f\left(q^{-1 / 2} z\right)$ (see for instance [GR04]). The standard $q$-exponentials do satisfy analogous $q$-difference equations, but, unlike them, the symmetric $q$-exponential satisfies

$$
\exp _{q}(z)=\exp _{q^{-1}}(z)
$$

which is a natural symmetry in the flat problem (see Remark 2.2.v). 
2.2. Fredholm Pfaffian formula. Now we can state one of our main results. We need to introduce some functions (in all that follows we fix a parameter $\beta>0$ ): for $s \in \mathbb{C}, \lambda \in \mathbb{R}_{\geq 0}$, $y \in \mathbb{C}$ with $|y|=1$ and $\zeta \in \mathbb{C} \backslash \mathbb{R}_{>0}$, define

$$
\psi(s, \lambda, y ; \zeta)=(-\zeta)^{s} \frac{\tau^{-\beta s^{2}+\frac{3}{4} s-1}}{y} \frac{1-\tau^{s / 2} y}{1+\tau^{s / 2} y} \frac{\left(-\tau^{-s / 2} y ; \tau\right)_{\infty}}{\left(-\tau^{s / 2} y ; \tau\right)_{\infty}} e^{-\lambda \frac{1-\tau^{s / 2} y}{1+\tau^{s / 2} y}+t\left[\frac{1}{1+\tau^{-s / 2} y}-\frac{1}{1+\tau^{s / 2} y}\right]}
$$

and let, for $\omega \in \mathbb{R}$,

$$
\check{\psi}(\omega, \lambda, y ; \zeta)=\frac{1}{2 \pi \mathrm{i}} \int_{\mathrm{i} \mathbb{R}} d s e^{s \omega} \psi(s, \lambda, y ; \zeta),
$$

which should be regarded as the inverse double-sided Laplace transform of $\psi$ (in $s$ ), see (5.7); additionally, if $z, z_{1}, z_{2} \in \mathbb{R}$ and $\sigma, \sigma_{1}, \sigma_{2} \in\{-1,1\}$, let

$$
\begin{array}{rlrl}
F_{1}(z, y) & =\sum_{m=1}^{\infty}(-z)^{m}(1-\tau)^{2 m} \tau^{\left(\frac{1}{2}+2 \beta\right) m^{2}-m} \frac{1+y^{2}}{y^{2}-1} \frac{\left(\tau^{1+m} y^{2} ; \tau\right)_{\infty}\left(\tau^{1+m} / y^{2} ; \tau\right)_{\infty}}{\left(\tau y^{2} ; \tau\right)_{\infty}\left(\tau / y^{2} ; \tau\right)_{\infty}} \\
F_{2}\left(z_{1}, z_{2} ; \sigma_{1}, \sigma_{2}\right)= & \sum_{m_{1}, m_{2}=1}^{\infty}\left(-z_{1}\right)^{m_{1}}\left(-z_{2}\right)^{m_{2}}\left(-\sigma_{1} \sigma_{2}\right)^{m_{1} \wedge m_{2}+1} \operatorname{sgn}\left(\sigma_{2} m_{2}-\sigma_{1} m_{1}\right) \\
F_{3}(z) & =-\sum_{m=1}^{\infty} \frac{\tau^{\left(\frac{1}{4}+\beta\right) m^{2}-\frac{1}{4} m}}{m_{\tau} !}(-z)^{m} . & \times \frac{\tau^{\left(\frac{1}{4}+\beta\right)\left(m_{1}^{2}+m_{2}^{2}\right)-\frac{1}{4}\left(m_{1}+m_{2}\right)}}{\left(m_{1}\right)_{\tau} !\left(m_{2}\right)_{\tau} !}
\end{array}
$$

Theorem 2.1. For $\zeta \in \mathbb{C}$ with $|\zeta|<\tau^{1 / 4}$, we have

$$
\mathbb{E}^{\text {flat }}\left[\exp _{\tau}\left(\zeta \tau^{\frac{1}{2} h(t, 0)}\right)\right]=\operatorname{Pf}\left[J-\widetilde{K}^{\text {flat }, \zeta}\right]_{L^{2}\left(\mathbb{R}_{\geq 0}\right)}
$$

where the $2 \times 2$ matrix kernel $\widetilde{K}^{\text {flat }}$ is given in terms of the functions defined in (2.8) and (2.10), with any $\beta>0$, through (here $C_{0,1}$ is the unit circle centered at the origin)

$$
\begin{aligned}
\widetilde{K}_{1,1}^{\text {flat }, \zeta}\left(\lambda_{1}, \lambda_{2}\right)= & \int_{\mathbb{R}^{2}} d \omega_{1} d \omega_{2} \frac{1}{\pi \mathrm{i}} \int_{C_{0,1}} d y \check{\psi}\left(\omega_{1}, \lambda_{1}, y\right) \check{\psi}\left(\omega_{2}, \lambda_{2}, \frac{1}{y}\right) F_{1}\left(e^{-\left(\omega_{1}+\omega_{2}\right)}, y\right) \\
& +\frac{1}{2} \sum_{\sigma_{1}, \sigma_{2} \in\{-1,1\}} \int_{\mathbb{R}^{2}} d \omega_{1} d \omega_{2} \check{\psi}\left(\omega_{1}, \lambda_{1}, \sigma_{1}\right) \check{\psi}\left(\omega_{2}, \lambda_{2}, \sigma_{2}\right) F_{2}\left(e^{-\omega_{1}}, e^{-\omega_{2}} ; \sigma_{1}, \sigma_{2}\right) \\
\widetilde{K}_{1,2}^{\text {flat }, \zeta}\left(\lambda_{1}, \lambda_{2}\right)= & \frac{1}{2} \sum_{\sigma \in\{-1,1\}} \int_{-\infty}^{\infty} d \omega F_{3}\left(e^{-\omega}\right) \check{\psi}\left(\omega ; \lambda_{1}, \sigma\right) \\
\widetilde{K}_{2,2}^{\text {flat }, \zeta}\left(\lambda_{1}, \lambda_{2}\right)= & \frac{1}{2} \operatorname{sgn}\left(\lambda_{2}-\lambda_{1}\right) .
\end{aligned}
$$

\section{Remark 2.2.}

(i) The symbol $f$ in the $y$ integral appearing in the definition of $\widetilde{K}_{1,1}^{\text {flat }}$ denotes that this is a principal value integral. This is because the $y$ contour goes through the singularities of $F_{1}(z, y)$ at $y= \pm 1$ (see the discussion starting after (4.20)).

(ii) The parameter $\beta>0$ which appears in (2.8) and (2.10) is necessary to make (2.9) a convergent integral, but otherwise has no meaning.

(iii) The three functions in (2.10) should be regarded as certain $q$-deformations of classical special functions. For example, $F_{3}(z)-1$ is a $q$-deformation of the exponential; in fact, $F_{3}(z)=1-\exp _{\tau}(-z)$ if $\beta=0$. Similarly, $F_{1}$ is related to the Bessel function $J_{0}$ :

$$
F_{1}(z, y)=\frac{1+y^{2}}{y^{2}-1}\left(J_{0}\left(2 \sqrt{z} ; \tau, y^{2}\right)-1\right)
$$


where $J_{0}(z ; \tau, y)$ is a 2-parameter $(\tau \in(0,1), y \in \mathbb{C}$ with $|y|=1)$ deformation of the Bessel function given by $J_{0}(z ; \tau, y)=\sum_{m=0}^{\infty}(1-\tau)^{2 m} \tau^{\left(\frac{1}{2}+2 \beta\right) m^{2}-m} \frac{\left(-z^{2} / 4\right)^{m}}{\left(\tau y^{2} ; \tau\right)_{m}\left(\tau / y^{2} ; \tau\right)_{m}}$. As $y \rightarrow \pm 1, J_{0}(z ; \tau, y)$ becomes $\widetilde{J}_{0}^{(1)}(z ; \tau)=\sum_{m=0}^{\infty} \tau^{\left(\frac{1}{2}+2 \beta\right) m^{2}-m} \frac{\left(-z^{2} / 4\right)^{m}}{\left(m_{\tau} !\right)_{m}^{2}}$, which in the case $\beta=0$ can be regarded as a symmetric version of the Jackson $q$-Bessel function $J_{0}^{(1)}(z ; \tau)=\sum_{m=0}^{\infty} \frac{\left(-z^{2} / 4\right)^{m}}{(1-\tau)^{2 m}\left(m_{\tau} !\right)_{m}^{2}}$ (which, as $\tau \rightarrow 1$ as well, becomes the standard Bessel function $\left.J_{0}(z)\right) . F_{2}$ is a bit more difficult to recognize, so we will not attempt it here, but in view of (146) in [LDC12] it should be possible to write it in terms of certain $q$-deformations of the Bessel and hyperbolic sine functions.

(iv) The statistical symmetry of flat ASEP under the transformation $(p, q) \mapsto(q, p)$ together with $h \mapsto-h$ is respected by the formula because the symmetric $q$-exponential satisfies (2.7).

(v) There are similar, although less appealing, formulas for generating functions of $\tau^{\frac{1}{2} h(0, x)}$ defined in terms of other $q$-deformations of the exponential function, see Theorem 5.1. These include formulas for the case of $E_{\tau}$, but not $e_{\tau}$. This last case does not seem to be accessible through our methods, because the moments of $\tau^{\frac{1}{2} h(0, x)}$ are expected to grow like $e^{c k^{2}}$ for some $c>0$, and hence $\mathbb{E}^{\text {flat }}\left[e_{\tau}\left(\zeta \tau^{\frac{1}{2} h(t, 0)}\right)\right]$ cannot be computed by summing moments. It is worth emphasizing here that the use of the function $\exp _{\tau}(z)$ is intrinsic to the problem, and not just a convenient choice. The formulas for other generating functions are not Fredholm Pfaffians.

(vi) The $\exp _{\tau}$ generating function on the left hand side of (2.11) does not in general determine the distribution of $h(t, 0)$. On the other hand, the formula in [Lee10] is for the distribution function of $h(t, 0)$, but it is not obvious how to obtain (2.11) from Lee's formula.

(vii) Focusing on the left hand side of (2.11), it is tempting to believe that $\exp _{\tau}(z)$ behaves sufficiently like $\exp (z)$ so that the key identity

$$
\lim _{t \rightarrow \infty} \mathbb{E}^{\text {flat }}\left[\exp \left\{-e^{\alpha\left(h(t, 0)-\frac{1}{2} t-t^{1 / 3} r\right)}\right\}\right]=\mathbb{P}^{\text {flat }}\left(\lim _{t \rightarrow \infty} \frac{h(t, 0)-\frac{1}{2} t}{t^{1 / 3}}>r\right)
$$

for $\alpha<0$ still holds with exp replaced by $\exp _{\tau}$. Indeed, this is the case with $e_{\tau}$ (this fact has been used succesfully in recent years to derive the asymptotics of some related models, although not for the half-flat or flat initial conditions, see for instance [BC14; BCF12; FV13]).

For $x>-(1-\tau)^{-1}$ it is the case that $\exp _{\tau}(z)$ looks quite like $\exp (z)$, and in fact it converges to it uniformly on $[-a, \infty)$ for any $a>0$, as $\tau \nearrow 1$. However, $\exp _{\tau}(z)$ has a largest real zero at $x_{0} \sim-(1-\tau)^{-1}$, and as $x$ decreases below $x_{0}$, it begins to oscillate wildly, with zeros at $x_{k} \in\left[x_{0} q^{-(k+1) / 2}, x_{0} q^{-k / 2}\right]$ for each $k \in \mathbb{Z}_{\geq 1}$ - their precise disposition is unknown [NG94] - and reaching size approximately $e^{|\log | x||^{2} / \log (1-\tau) \mid}$ in between. Unfortunately, this genuinely precludes estimations cutting off this bad region.

(viii) A formal steepest descent analysis shows that, setting $\zeta=-\tau^{-t / 4+t^{1 / 3} r / 2}$, the right hand side of (2.11) leads in the long time limit, as expected, to the GOE TracyWidom distribution. The limit is obtained in the form

$$
F_{\mathrm{GOE}}(r)=\operatorname{Pf}\left[J-K_{r}\right]_{L^{2}([0, \infty))}
$$

with

$$
K_{r}\left(\lambda_{1}, \lambda_{2}\right)=\left[\begin{array}{cc}
\frac{1}{2}\left(\partial_{\lambda_{1}}-\partial_{\lambda_{2}}\right) K_{\mathrm{Ai}}\left(\lambda_{1}+r, \lambda_{2}+r\right) & -\frac{1}{2} \operatorname{Ai}\left(\lambda_{1}+r\right) \\
\frac{1}{2} \operatorname{Ai}\left(\lambda_{2}+r\right) & \frac{1}{2} \operatorname{sgn}\left(\lambda_{2}-\lambda_{2}\right)
\end{array}\right],
$$

a formula for the Tracy-Widom GOE distribution which is essentially equivalent to one implicit in [LDC12], and which is also very similar to (but not quite the same 
as) a formula appearing in [Fer04]. Here the Airy kernel $K_{\mathrm{Ai}}$ is defined as

$$
K_{\mathrm{Ai}}\left(\lambda_{1}, \lambda_{2}\right)=\int_{0}^{\infty} d \xi \operatorname{Ai}\left(\lambda_{1}+\xi\right) \operatorname{Ai}\left(\lambda_{2}+\xi\right)
$$

A proof of (2.14) together with the formal $t \rightarrow \infty$ asymptotic analysis leading to this Fredholm Pfaffian is given in Appendix A.

(ix) Although we have not included it, a similar formal asymptotic analysis in the weakly asymmetric limit leads to the Le Doussal-Calabrese Pfaffian formula for flat KPZ [LDC12] (see also Section 6 for related formulas).

2.3. Moment formula and emergence of the Pfaffian structure. The sum in (2.6) includes a brutal cutoff by $q^{n^{2} / 4}$, so that the left hand side of (2.11) is practically a finite sum of moments. Thus, in a sense, the Pfaffian formula is mostly indicating a nice algebraic structure for a sum of moments. Therefore we state separately our moment formula. It involves a kernel $K^{\text {flat }}$ which is related to, but not quite the same as the kernel $\widetilde{K}^{\text {flat, } \zeta}$ appearing in (2.11). Since the meaning of the terms in $K^{\text {flat }}$ only becomes apparent in the computations, we do not repeat the detailed formula here.

Theorem 2.3. Let $K^{\text {flat }}$ be the the $2 \times 2$ matrix kernel given by (4.24) and (4.26). Then for any $m \in \mathbb{Z}_{\geq 0}$ we have

$$
\mathbb{E}^{\mathrm{flat}}\left[\tau^{\frac{1}{2} m h(t, 0)}\right]=m_{\tau} ! \tau^{-\frac{1}{4} m^{2}} \sum_{k=0}^{m} \frac{(-1)^{k}}{k !} \sum_{\substack{m_{1}, \ldots, m_{k}=1, m_{1}+\cdots+m_{k}=m}}^{\infty} \int_{\left(\mathbb{R}_{\geq 0}\right)^{k}} d \vec{\lambda} \operatorname{Pf}\left[K^{\mathrm{flat}}\left(\lambda_{a}, \lambda_{b} ; m_{a}, m_{b}\right)\right]_{a, b=1}^{k} .
$$

The route to these moment formulas for the flat initial data is by taking appropriate limits of formulas for the half-flat initial case (2.3). The limit we are interested in consists in starting ASEP with the shifted half-flat initial condition $\eta_{0}(y)=\mathbf{1}_{y \in 2 \mathbb{Z}_{>-x}}$, considering the variable $h(t, 0)$ and computing the limit when $x \rightarrow \infty$. More precisely, we will obtain moment formulas for flat ASEP through the identity

$$
\mathbb{E}^{\text {flat }}\left[\tau^{\frac{1}{2} m h(t, 0)}\right]=\lim _{x \rightarrow \infty} \mathbb{E}^{2 \mathbb{Z}>-x}\left[\tau^{\frac{1}{2} m h(t, 0)}\right]
$$

for $m \geq 0$, where the superscript on the right hand side simply refers to the initial condition specified above. Introduce now the random variables

$$
N_{x}(t)=\sum_{y=-\infty}^{x} \eta_{t}(y)
$$

which are of course finite if and only if the initial condition is left-finite. It is not hard to check that when all particles start to the right of the origin one has $N_{0}^{\text {flux }}(t)=N_{0}(t)$, and hence from (2.1) we get

$$
h(t, x)=2 N_{x}(t)-x
$$

in the half-flat case. On the other hand a simple coupling argument shows that $h(t, 0)$, with initial condition $\eta_{0}(y)=\mathbf{1}_{y \in 2 \mathbb{Z}_{>-x}}$ has the same distribution as the shifted observable $h(t, 2 x)$ with initial condition $\eta_{0}(y)=\mathbf{1}_{y \in 2 \mathbb{Z}_{>0}}$. Hence, using (2.18) and (2.19) we deduce that

$$
\mathbb{E}^{\text {flat }}\left[\tau^{\frac{1}{2} m h(t, 0)}\right]=\lim _{x \rightarrow \infty} \mathbb{E}^{\mathrm{h}-\mathrm{f}}\left[\tau^{m\left(N_{2 x}(t)-x\right)}\right] .
$$

In Theorem 1.3 of [OQR14] we obtained the following formula for the moments appearing on the right hand side of (2.20):

Theorem 2.4. Let $m \geq 0$. Then

$$
\mathbb{E}^{\mathrm{h}-\mathrm{fl}}\left[\tau^{m N_{x}(t)}\right]=m_{\tau} ! \sum_{k=0}^{m} \nu_{k, m}^{\mathrm{h}-\mathrm{fl}}(t, x)
$$


with

$$
\begin{aligned}
\nu_{k, m}^{\mathrm{h}-\mathrm{fl}}(t, x)=\frac{1}{k !} \sum_{\substack{n_{1}, \ldots, n_{k} \geq 1 \\
n_{1}+\cdots+n_{k}=m}} \frac{1}{(2 \pi \mathrm{i})^{k}} \int_{C_{0, \tau}^{k}-\eta} d \vec{w} \operatorname{det}\left[\frac{-1}{w_{a} \tau^{n_{a}}-w_{b}}\right]_{a, b=1}^{k} \\
\quad \times \prod_{a=1}^{k} \mathfrak{f}\left(w_{a} ; n_{a}\right) \mathfrak{g}_{\mathrm{p}}\left(w_{a} ; n_{a}\right) \prod_{1 \leq a<b \leq k} \mathfrak{h}_{1}\left(w_{a}, w_{b} ; n_{a}, n_{b}\right),
\end{aligned}
$$

where $C_{0, \tau^{-\eta}}$ is a circle of radius $\tau^{-\eta}$ centered at the origin, with $\eta \in(0,1 / 4), C_{0, \tau^{-\eta}}^{k}$ denotes the product of $k$ copies of $C_{0, \tau^{-\eta}}$, and where

$$
\mathfrak{f}(w ; n)=(1-\tau)^{n} e^{(q-p) t\left[\frac{1}{1+w}-\frac{1}{1+\tau^{n} w}\right]}\left(\frac{1+\tau^{n} w}{1+w}\right)^{x-1},
$$

and $\mathfrak{g}_{\mathrm{p}}$ and $\mathfrak{h}_{1}$ are given in $(2.23)$.

For simplicity, throughout the rest of the paper we will omit the bound on the indices in products such as $1 \leq a \leq k$ and $1 \leq a<b \leq k$ when no confusion can arise and the factors involved in the products are defined in terms of a collection of $k$ variables. A similar convention will sometimes be used for sums. Additionally, we will continue using the notation $C^{k}$ for the product of $k$ copies of a given contour $C$ in the complex plane.

One of the most interesting parts of the story is the computation of the limit (2.20), which involves taking the limit $x \rightarrow \infty$ of the right hand side of (2.22) with $x$ replaced by $2 x$ and after multiplying by $\tau^{-m x}$. We will start by rewriting this formula in a slightly different way which will be better adapted for the calculation of this limit. Introduce the following functions:

$$
\begin{gathered}
\mathfrak{f}_{1}(w ; n)=\frac{(1-\tau)^{n}}{w\left(1-\tau^{n}\right)} e^{(q-p) t\left[\frac{1}{1+w}-\frac{1}{1+\tau^{n} w}\right]}, \quad \mathfrak{f}_{2}(w ; n)=\left(\frac{1+\tau^{n} w}{1+w} \tau^{-\frac{1}{2} n}\right)^{2 x-1}, \\
\mathfrak{g}_{\mathrm{p}}(w ; n)=\frac{(-w ; \tau)_{\infty}}{\left(-\tau^{n} w ; \tau\right)_{\infty}} \frac{\left(\tau^{2 n} w^{2} ; \tau\right)_{\infty}}{\left(\tau^{n} w^{2} ; \tau\right)_{\infty}} \\
\mathfrak{g}_{\mathrm{u}}\left(w_{a} ; n_{a}\right)=\frac{\left(-w_{a} ; \tau\right)_{\infty}}{\left(-\tau^{n_{a}} w_{a} ; \tau\right)_{\infty}} \frac{\left(\tau^{2 n_{a}} w_{a}^{2} ; \tau\right)_{\infty}}{\left(\tau^{1+n_{a}} w_{a}^{2} ; \tau\right)_{\infty}} \\
\mathfrak{h}_{1}\left(w_{1}, w_{2} ; n_{1}, n_{2}\right)=\frac{\left(w_{1} w_{2} ; \tau\right)_{\infty}\left(\tau^{n_{1}+n_{2}} w_{1} w_{2} ; \tau\right)_{\infty}}{\left(\tau^{n_{1}} w_{1} w_{2} ; \tau\right)_{\infty}\left(\tau^{n_{2}} w_{1} w_{2} ; \tau\right)_{\infty}} \\
\mathfrak{h}_{2}\left(w_{a}, w_{b} ; n_{a}, n_{b}\right)=\frac{\left(w_{a} \tau^{n_{a}}-w_{b} \tau^{n_{b}}\right)\left(w_{b}-w_{a}\right)}{\left(w_{a} \tau^{n_{a}}-w_{b}\right)\left(w_{b} \tau^{n_{b}}-w_{a}\right)}
\end{gathered}
$$

$\mathfrak{g}_{\mathrm{u}}$ will not appear in the coming formula (2.26), but will appear later in Theorem 2.5. The subscripts in $\mathfrak{g}_{\mathrm{p}}$ and $\mathfrak{g}_{\mathrm{u}}$ stand for "paired" and "unpaired", a terminology which we will explain shortly and which will become clear in Section 3. Using these definitions in (2.22) (with $x$ replaced by $2 x$, and multiplied by $\tau^{-m x}$ ) and expanding the determinant in that formula using the Cauchy determinant formula

$$
\operatorname{det}\left[\frac{1}{x_{a}-y_{b}}\right]_{a, b=1}^{k}=\frac{\prod_{a<b}\left(x_{a}-x_{b}\right)\left(y_{b}-y_{a}\right)}{\prod_{a, b}\left(x_{a}-y_{b}\right)},
$$

we obtain

$$
\mathbb{E}^{\mathrm{h}-\mathrm{fl}}\left[\tau^{m\left(N_{2 x}(t)-x\right)}\right]=m_{\tau} ! \sum_{k=0}^{m} \widetilde{\nu}_{k, m}^{\mathrm{h}-\mathrm{fl}}(t, 2 x)
$$


with

$$
\begin{array}{r}
\widetilde{\nu}_{k, m}^{\mathrm{h}-\mathrm{fl}}(t, 2 x)=\frac{1}{k !} \sum_{\substack{n_{1}, \ldots, n_{k} \geq 1 \\
n_{1}+\cdots+n_{k}=m}} \frac{1}{(2 \pi \mathrm{i})^{k}} \int_{C_{0, \tau}^{k}-\eta} d \vec{w} \prod_{a} \mathfrak{f}_{1}\left(w_{a} ; n_{a}\right) \mathfrak{f}_{2}\left(w_{a} ; n_{a}\right) \mathfrak{g}_{\mathrm{p}}\left(w_{a} ; n_{a}\right) \\
\times \prod_{a<b} \mathfrak{h}_{1}\left(w_{a}, w_{b} ; n_{a}, n_{b}\right) \mathfrak{h}_{2}\left(w_{a}, w_{b} ; n_{a}, n_{b}\right) .
\end{array}
$$

We have added a tilde in $\widetilde{\nu}_{k, m}^{\mathrm{h}-\mathrm{fl}}(t, 2 x)$ to indicate the fact that we have multiplied by $\tau^{-m x}$ in (2.25) (in other words, $\widetilde{\nu}_{k, m}^{\mathrm{h}-\mathrm{fl}}(t, 2 x)=\tau^{-m x} \nu_{k, m}^{\mathrm{h}-\mathrm{fl}}(t, 2 x)$ in view of $(2.21)$ ). Note that in (2.26) we have written $\tau^{-m x}$ as $\prod_{a} \tau^{-n_{a} x}$.

Note that the only factors in the integrand on the right hand side of (2.26) that depend on $x$ are those of the form $\mathfrak{f}_{2}\left(w_{a} ; n_{a}\right)$. An easy computation shows that the base in this power has modulus strictly less than 1 if and only if $\left|w_{a}\right|>\tau^{-n_{a} / 2}$. This suggests that for each $a=1, \ldots, k$ we should deform the corresponding contour $C_{0, \tau^{-\eta}}$ to some contour lying just outside the ball of radius $\tau^{-n_{a} / 2}$.

As we perform this deformation we will cross many poles. The residue calculus associated to this deformation is quite complicated, and is explained in detail in Sections 3.1 and 3.2. The result has two properties which turn out to be crucial for the sequel. First, there are two types of poles that are crossed (see the list in page 11): the ones coming from $\mathfrak{g}_{\mathrm{p}}\left(w_{a}, n_{a}\right)$, which occur at $w_{a}= \pm \tau^{-n_{a} / 2}$, and the ones coming from $\mathfrak{h}_{1}\left(w_{a}, w_{b} ; n_{a}, n_{b}\right)$, which occur at $w_{b}=\tau^{-n_{a}} / w_{a}$ whenever $n_{a}=n_{b}$. We will refer to these two types of poles respectively as unpaired poles and paired poles. This pairing structure is the key to the emergence of the Pfaffian.

The second crucial property is that the residues of the unpaired and paired poles are such that $x$ disappears from the corresponding factors (see (3.2) and (3.4)). As a consequence of this, after deforming the contours $x$ only appears in factors of the form $\mathfrak{f}_{2}\left(w_{a} ; n_{a}\right)$ with $w_{a}$ living in the deformed contour, which are such that $\left|\mathfrak{f}_{2}\left(w_{a} ; n_{a}\right)\right| \rightarrow 0$ as $x \rightarrow \infty$. This will allow us to derive an exact formula for the limit of $\widetilde{\nu}_{k, m}^{\mathrm{h}-\mathrm{fl}}(t, 2 x)$, which after further simplification and a change of variables which turns the contours into circles of radius 1 (see Section 3.3), leads to the result that follows. To state it we define the functions

$$
\begin{gathered}
\mathfrak{e}\left(w_{1}, w_{2} ; n_{1}, n_{2}\right)=\frac{\left(1-\tau^{n_{1}} w_{1} w_{2}\right)\left(1-\tau^{n_{2}} w_{1} w_{2}\right)}{\left(1-w_{1} w_{2}\right)\left(1-\tau^{n_{1}+n_{2}} w_{1} w_{2}\right)} \\
\mathfrak{h}\left(w_{1}, w_{2} ; n_{1}, n_{2}\right)=\mathfrak{h}_{1}\left(w_{1}, w_{2} ; n_{1}, n_{2}\right) \mathfrak{h}_{2}\left(w_{1}, w_{2} ; n_{1}, n_{2}\right) .
\end{gathered}
$$

Furthermore, given generic functions $a(z, n)$ and $b\left(z_{1}, z_{2} ; n_{1}, n_{2}\right)$, we define modified functions $\tilde{a}$ and $\tilde{b}$ through

$$
\tilde{a}(z, n)=a\left(\tau^{-\frac{1}{2} n} z, n\right) \quad \text { and } \quad \tilde{b}\left(z_{1}, z_{2} ; n_{1}, n_{2}\right)=b\left(\tau^{-\frac{1}{2} n_{1}} z_{1}, \tau^{-\frac{1}{2} n_{2}} z_{2} ; n_{1}, n_{2}\right)
$$

In the formula that follows this modifier will be applied to all the functions defined in (2.23) and (2.27) (the modification arises through the change of variables performed at the end of Section 3.3, see (3.18) and (3.19)).

Theorem 2.5. Let

$$
\nu_{k, m}^{\mathrm{flat}}(t)=\lim _{x \rightarrow \infty} \widetilde{\nu}_{k, m}^{\mathrm{h}-\mathrm{fl}}(t, 2 x) .
$$


Then, with the notation introduced in (2.23), (2.27) and (2.28), and letting $C_{0,1}$ be a circle of radius 1 centred at the origin,

$$
\begin{aligned}
& \nu_{k, m}^{\text {flat }}(t)=\sum_{\substack{k_{\mathrm{u}}, k_{\mathrm{p}} \geq 0 \\
k_{\mathrm{u}}+2 k_{\mathrm{p}}=k}} \frac{1}{k_{\mathrm{u}} ! 2^{k_{\mathrm{p}}} k_{\mathrm{p}} !} \sum_{\sigma_{1}, \ldots, \sigma_{k_{\mathrm{u}}} \in\{-1,1\}} \sum_{\substack{n_{1}^{\mathrm{u}}, \ldots, n_{k_{\mathrm{u}}^{\mathrm{u}}}^{\mathrm{u}} \geq 1 \\
\sigma_{a} n_{a}^{\mathrm{u}} \neq \sigma_{b} n_{b}^{\mathrm{u}}, a \neq b}} \sum_{n_{1}^{\mathrm{p}}, \ldots, n_{k_{\mathrm{p}}}^{\mathrm{p}} \geq 1} \mathbf{1}_{\sum_{a} n_{a}^{\mathrm{u}}+2 \sum_{a} n_{a}^{\mathrm{p}}=m} \\
& \times \frac{1}{(2 \pi \mathrm{i})^{k}} \int_{C_{0,1}^{k_{\mathrm{p}}}} d \vec{w}^{\mathrm{p}} \prod_{a=1}^{k_{\mathrm{u}}}\left(\tilde{\mathfrak{f}}_{1} \tilde{\mathfrak{g}}_{\mathrm{u}}\right)\left(\sigma_{a} ; n_{a}\right) \prod_{a=1}^{k_{\mathrm{p}}}\left(\tilde{\mathfrak{f}}_{1} \tilde{\mathfrak{g}}_{\mathrm{p}}\right)\left(z_{a}^{\mathrm{p}} ; n_{a}^{\mathrm{p}}\right)\left(\tilde{\mathfrak{f}}_{1} \tilde{\mathfrak{g}}_{\mathrm{p}}\right)\left(\frac{1}{z_{a}^{\mathrm{p}}} ; n_{a}^{\mathrm{p}}\right) \\
& \times \prod_{a=1}^{k_{\mathrm{u}}} \frac{1}{2} \sigma_{a} \tau^{1-\frac{1}{2} n_{a}^{\mathrm{u}}} \prod_{a=1}^{k_{\mathrm{p}}} \frac{(-1)^{n_{a}^{\mathrm{p}}}}{z_{a}^{\mathrm{p}}} \tau^{2-\frac{1}{2} n_{a}^{\mathrm{p}}\left(n_{a}^{\mathrm{p}}+1\right)}\left(\tau^{-n_{a}^{\mathrm{p}}}\right) \tilde{\mathfrak{h}}_{2}\left(z_{a}^{\mathrm{p}}, \frac{1}{z_{a}^{\mathrm{p}}} ; n_{a}^{\mathrm{p}}, n_{a}^{\mathrm{p}}\right) \\
& \times \prod_{1 \leq a<b \leq k_{\mathrm{u}}} \tilde{\mathfrak{h}}\left(\sigma_{a}, \sigma_{b} ; n_{a}^{\mathrm{u}}, n_{b}^{\mathrm{u}}\right) \prod_{a \leq k_{\mathrm{u}}, b \leq k_{\mathrm{p}}} \tau^{-n_{a}^{\mathrm{u}} n_{b}^{\mathrm{p}} \tilde{\mathfrak{e}}}\left(\sigma_{a}, z_{b}^{\mathrm{p}} ; n_{a}^{\mathrm{u}}, n_{b}^{\mathrm{p}}\right) \tilde{\mathfrak{e}}\left(\sigma_{a}, \frac{1}{z_{b}^{\mathrm{p}}} ; n_{a}^{\mathrm{u}}, n_{b}^{\mathrm{p}}\right)
\end{aligned}
$$

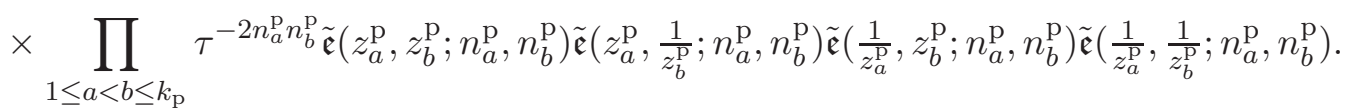

In view of (2.20) and (2.25), this provides a first formula for $\mathbb{E}^{\text {flat }}\left[\tau^{m h(t, 0) / 2}\right]$, which will be later turned into (2.17). A minor issue with this formula is that the numerator and denominator in the factors of the form $\tilde{\mathfrak{h}}\left(\sigma_{a}, \sigma_{b} ; n_{a}^{\mathrm{u}}, n_{b}^{\mathrm{u}}\right)$ vanish when $\sigma_{a} \sigma_{b}=1$ and $n_{a}^{\mathrm{u}}-n_{b}^{\mathrm{u}}$ is even, in which case the factor is to be interpreted as in (3.5) below (see the discussion preceding that definition).

This formula can be regarded as a microscopic (and rigorous) version of formulas (107)(108) of [LDC12], which can be recovered in the weakly asymmetric limit (see also Section $6)$.

To get an idea of how the pairing structure arising from the contour deformation leads to a Pfaffian formula for $\nu_{k, m}^{\text {flat }}(t)$, let us consider first the product

$$
\prod_{1 \leq a<b \leq k_{\mathrm{p}}} \tilde{\mathfrak{e}}\left(z_{a}^{\mathrm{p}}, z_{b}^{\mathrm{p}} ; n_{a}^{\mathrm{p}}, n_{b}^{\mathrm{p}}\right) \tilde{\mathfrak{e}}\left(z_{a}^{\mathrm{p}}, z_{b}^{-\mathrm{p}} ; n_{a}^{\mathrm{p}}, n_{b}^{\mathrm{p}}\right) \tilde{\mathfrak{e}}\left(z_{a}^{-\mathrm{p}}, z_{b}^{\mathrm{p}} ; n_{a}^{\mathrm{p}}, n_{b}^{\mathrm{p}}\right) \tilde{\mathfrak{e}}\left(z_{a}^{-\mathrm{p}}, z_{b}^{-\mathrm{p}} ; n_{a}^{\mathrm{p}}, n_{b}^{\mathrm{p}}\right) .
$$

It turns out that this product has the structure of a Schur Pfaffian. To see this, we recall first Schur's Pfaffian identity:

$$
\prod_{1 \leq a<b \leq 2 k} \frac{x_{a}-x_{b}}{x_{a}+x_{b}}=\operatorname{Pf}\left[\frac{x_{a}-x_{b}}{x_{a}+x_{b}}\right]_{a, b=1}^{2 k} .
$$

Letting $y_{a}=\frac{x_{a}-1}{x_{a}+1}$ we obtain the identity

$$
\operatorname{Pf}\left[\frac{y_{b}-y_{a}}{y_{a} y_{b}-1}\right]_{a, b=1}^{2 k}=\prod_{1 \leq a<b \leq 2 k} \frac{y_{b}-y_{a}}{y_{a} y_{b}-1} .
$$

Although the four factors inside the product in (2.30) are not of the form $\left(y_{b}-y_{a}\right) /\left(y_{a} y_{b}-1\right)$, this expression appears after rearranging the factors in the numerator and denominator. In fact, letting

$$
y_{2 a-1}=z_{a}^{\mathrm{p}} \quad \text { and } \quad y_{2 a}=z_{a}^{-\mathrm{p}}
$$

for $a=1, \ldots, k_{\mathrm{p}}$, one checks that the product in $(2.30)$ is given exactly by $\prod_{a<b} \frac{y_{b}-y_{a}}{y_{a} y_{b}-1}$, and thus it equals the Pfaffian in (2.32).

In Section 4 we will show how the last two lines of (2.29) (which include in particular the product in (2.30)) can be turned into the product of two Pfaffians, times the products of the factors of the form $\tau^{-n_{a}^{\mathrm{u}} n_{b}^{\mathrm{p}}}$ and $\tau^{-2 n_{a}^{\mathrm{p}} n_{b}^{\mathrm{p}}}$. We will then show how the two types of variables (paired and unpaired) can be put on the same footing, and finally how the whole integrand in (2.29) can (almost) be written as single Pfaffian. The result is (2.17). From 
this we can form a generating function. This is done in Theorem 5.2, a particular case of which becomes, after further manipulations, Theorem 2.1.

The Pfaffian structure which we have described was first discovered in the context of the delta Bose gas with flat initial data in [LDC12], using a rough singularity analysis. They started from the divergent series for the generating function, while we start from moment formulas (4.18) which are more reminiscent of the Borodin-Corwin approach [BC14]. In hindsight, the two give rather similar results. However, the existence of nice exact expressions for the flat case at the ASEP level is a surprise, and it is far from clear that it is replicated in other solvable models such as $q$-TASEP, the log-Gamma polymer, and the O'Connell-Yor semi-discrete polymer.

We next describe in detail the core of the argument, which is the residue summation.

\section{Moment formula: CONTOUR Deformation}

\section{And though the poles were rather small}

They had to count them all

The goal of this section is to prove Theorem 2.5. We are trying to compute the limit as $x \rightarrow \infty$ of $\widetilde{\nu}_{k, m}^{\mathrm{h}-\mathrm{fl}}$, where $\widetilde{\nu}_{k, m}^{\mathrm{h}-\mathrm{fl}}$ is defined in (2.26). As we noted in the introduction, the only factor in the integrand on the right hand side of (2.26) that depends on $x$ is $\mathfrak{f}_{2}\left(w_{a}, n_{a}\right)$, and the base in this power is strictly bounded by 1 in modulus whenever $\left|w_{a}\right|>\tau^{-n_{a} / 2}$. Hence in order to compute the limit, we will deform the $w_{a}$ contours to some contour which has this property, which for simplicity we choose to be the contour $C_{0}^{n_{a}}$ given by a circle of radius $\tau^{-n_{a} / 2-\eta}$ centered at 0 .

3.1. Poles of the integrand. In order to write down the result of the contour deformation we need to determine first what poles are crossed as we deform each of the contours. It will be useful to list first the possible singularities of the integrand:

(a) $\mathfrak{f}_{1}\left(w_{a}, n_{a}\right)$ has a simple pole at $w_{a}=0$, but this point is inside the original contour $C_{0, \tau^{-\eta}}$. It also has essential singularities at $w_{a}=-1$ and $w_{a}=-\tau^{-n_{a}}$, but the first one is contained inside $C_{0, \tau^{-\eta}}$ while the second is outside $C_{0}^{n_{a}}$. So this factor will not contribute any poles.

(b) $\mathfrak{f}_{2}\left(w_{a}, n_{a}\right)$ has simple poles at $w_{a}=-1$ or $w_{a}=-\tau^{-n_{a}}$ (depending on the sign of $2 x-1)$, but as in (a) they are never in the deformation region.

(c) $\mathfrak{g}_{\mathrm{p}}\left(w_{a}, n_{a}\right)$ has simple poles at $w_{a}=-\tau^{-n_{a}-\ell}$ for any $\ell \in \mathbb{Z}_{\geq 0}$, but all these points lie outside $C_{0}^{n_{a}}$. It also has simple poles at $w_{a}= \pm \tau^{-n_{a} / 2-\ell / 2}$ for any $\ell \in \mathbb{Z}_{\geq 0}$. These points are inside the deformation region only when $\ell=0$, and we have, for $\sigma= \pm 1$,

$$
\operatorname{Res}_{w_{k}=\sigma \tau^{-\frac{1}{2} n_{k}}} \mathfrak{g}_{\mathrm{p}}\left(w_{k}, n_{k}\right)=-\frac{1}{2} \sigma \tau^{-\frac{1}{2} n_{k}} \mathfrak{g}_{\mathrm{u}}\left(\sigma \tau^{-\frac{1}{2} n_{k}}, n_{k}\right) .
$$

Observe also that, crucially, for $\sigma= \pm 1$,

$$
\mathfrak{f}_{2}\left(\sigma \tau^{-\frac{1}{2} n_{k}}, n_{k}\right)=\left(\frac{1+\sigma \tau^{n_{k} / 2}}{1+\sigma \tau^{-n_{k} / 2}} \tau^{-\frac{1}{2} n_{k}}\right)^{2 x-1}=\sigma .
$$

(d) The factors on the denominator of $\mathfrak{h}_{1}\left(w_{a}, w_{b} ; n_{a}, n_{b}\right)$ vanish when $w_{a} w_{b}=\tau^{-n_{a}-\ell_{a}}$ with $\ell_{a} \in \mathbb{Z}_{\geq 0}$ and $w_{a} w_{b}=\tau^{-n_{b}-\ell_{b}}$ with $\ell_{b} \in \mathbb{Z}_{\geq 0}$. Observe that whenever one of the two factors vanishes, the factor $\left(w_{a} w_{b} ; \tau\right)_{\infty}$ in the numerator of $\mathfrak{h}_{1}\left(w_{a}, w_{b} ; n_{a}, n_{b}\right)$ is going to vanish as well. Thus there can only be a (simple) pole when both factors on the denominator vanish at the same time, i.e., when $w_{b}=w_{a}^{-1} \tau^{-n_{a}-\ell_{a}}=w_{a}^{-1} \tau^{-n_{b}-\ell_{b}}$ for some $\ell_{a}, \ell_{b} \in \mathbb{Z}_{\geq 0}$. Whether these points lie inside the deformation region or not will depend on which stage of the deformation we are at:

(d.i) Assume first that $w_{a} \in C_{0, \tau^{-\eta}}$ and we are deforming the $w_{b}$ contour. The possible singularities occur at $w_{b}=w_{a}^{-1} \tau^{-n_{b}-\ell_{b}}$ for some $\ell_{b} \in \mathbb{Z}_{\geq 0}$. This point has modulus 
$\tau^{\eta-n_{b}-\ell_{b}} \geq \tau^{-\frac{1}{2} n_{b}-\eta}$ (recall $\left.\eta \in(0,1 / 4)\right)$ and thus it is outside the deformation region. As a consequence, this factor does not contribute a pole in this case.

(d.ii) Now assume that $w_{a} \in C_{0}^{n_{a}}$ as we deform the $w_{b}$ contour. There is a singularity when $w_{b}=\bar{w}_{1}=\bar{w}_{2}$ with $\bar{w}_{1}=w_{a}^{-1} \tau^{-n_{b}-\ell_{1}}$ and $\bar{w}_{2}=w_{a}^{-1} \tau^{-n_{a}-\ell_{2}}$. Observe that $\left|\bar{w}^{1}\right|=\tau^{n_{a} / 2+\eta-n_{b}-\ell_{1}}$ which is smaller than $\tau^{-n_{b} / 2-\eta}$ if and only if $\ell_{1} \leq$ $\left(n_{a}-n_{b}\right) / 2+2 \eta$. Similarly we have $\left|\bar{w}^{2}\right|=\tau^{-n_{a} / 2+\eta-\ell_{2}}$ which is smaller than $\tau^{-n_{b} / 2-\eta}$ if and only if $\ell_{2} \leq\left(n_{b}-n_{a}\right) / 2+2 \eta$. Remembering that we also need to have $\ell_{1}, \ell_{2} \geq 0$ these conditions on $\ell_{1}, \ell_{2}$ (and the fact that $\eta \in(0,1 / 4)$ ) imply that $n_{a}=n_{b}$, and thus also that $\ell_{1}=\ell_{2}=0$. Hence we have a simple pole at $w_{b}=w_{a}^{-1} \tau^{-n_{a}}$ when $n_{a}=n_{b}$, whose residue is computed to be

$$
\operatorname{Res}_{w_{b}=w_{a}^{-1} \tau^{-n_{a}}} \mathfrak{h}_{1}\left(w_{a}, w_{b} ; n_{a}, n_{a}\right)=\frac{(-1)^{n_{a}+1}}{w_{a}} \tau^{-n_{a}\left(n_{a}+1\right) / 2}\left(\tau^{-n_{a}}-1\right) .
$$

Note that, crucially, in the case $n_{a}=n_{b}$ we have

$$
\mathfrak{f}_{2}\left(w_{b}^{-1} \tau^{2-n_{b}}, n_{b}\right) \mathfrak{f}_{2}\left(w_{b}, n_{b}\right)=\left(\frac{\tau+w_{b}^{-1} \tau^{2}}{\tau+w_{b}^{-1} \tau^{2-n_{b}}} \frac{\tau+\tau^{n_{b}} w_{b}}{\tau+w_{b}} \tau^{-n_{b}}\right)^{x-1}=1 .
$$

(d.iii) The third possibility of interest is when $w_{a}=\sigma \tau^{-n_{a} / 2}$ for $\sigma= \pm 1$ (which happens when a residue in $w_{a}$ has already been evaluated as one of the poles from (c)). Exactly as in (d.ii), a singularity may only arise when $n_{a}=n_{b}$ at $w_{b}=\sigma \tau^{-n_{a} / 2}$. But in this case the factor $\mathfrak{h}_{2}\left(w_{b}, \sigma \tau^{-n_{a} / 2} ; n_{a}, n_{a}\right)$ has a double zero at this point, and hence the double zero in the denominator is canceled, which means that there is no pole in this case.

(e) Finally, the factors on the denominator of $\mathfrak{h}_{2}\left(w_{a}, w_{b} ; n_{a}, n_{b}\right)$ vanish when $w_{b}=w_{a} \tau^{n_{a}}$ and $w_{b}=w_{a} \tau^{-n_{b}}$. The first point is inside $C_{0, \tau^{-\eta}}$ whenever $w_{a}$ is in $C_{0, \tau^{-\eta}}$ or $C_{0}^{n_{a}}$. The second point is outside $C_{0}^{n_{b}}$ whenever $w_{a}$ is in $C_{0, \tau^{-\eta}}$ or $C_{0}^{n_{a}}$. So this factor will not contribute any poles.

Finally, consider the product $\left(\mathfrak{h}_{1} \mathfrak{h}_{2}\right)\left(w_{a}, \sigma_{b} \tau^{-n_{b} / 2} ; n_{a}, n_{b}\right)$ at $w_{a}=\sigma_{a} \tau^{-n_{a} / 2}$ for $\sigma_{a}, \sigma_{b}=$ \pm 1 , which occurs when both $w_{a}$ and $w_{b}$ are evaluated as residues coming from the poles in (c), with $w_{b}$ having been evaluated first. When $n_{a}-n_{b}$ is even and $\sigma_{a} \sigma_{b}=1$, both the numerator and the denominator of the factor coming from $\mathfrak{h}_{1}$ vanish. Since the evaluation at these points comes from a residue computation (in $w_{a}$ ), the product should be computed as

$$
\left(\mathfrak{h}_{1} \mathfrak{h}_{2}\right)\left(\sigma_{a} \tau^{-\frac{1}{2} n_{a}}, \sigma_{b} \tau^{-\frac{1}{2} n_{b}} ; n_{a}, n_{b}\right):=\lim _{w \rightarrow \sigma_{a} \tau^{-n_{a} / 2}}\left(\mathfrak{h}_{1} \mathfrak{h}_{2}\right)\left(w, \sigma_{b} \tau^{-\frac{1}{2} n_{b}} ; n_{a}, n_{b}\right) .
$$

The right hand side is computed in the next lemma. We stress that, in the formulas that will follow, we will use the extension of $\mathfrak{h}_{1} \mathfrak{h}_{2}$ defined in (3.5) mostly without reference.

Lemma 3.1. For $n_{a}, n_{b} \in \mathbb{Z}_{\geq 0}$ and $\sigma_{a}, \sigma_{b}= \pm 1$ we have

$$
\lim _{w \rightarrow \sigma_{a} \tau^{-n_{a} / 2}}\left(\mathfrak{h}_{1} \mathfrak{h}_{2}\right)\left(w, \sigma_{b} \tau^{-\frac{1}{2} n_{b}} ; n_{a}, n_{b}\right)=\left(-\sigma_{a} \sigma_{b}\right)^{n_{a} \wedge n_{b}} \tau^{-\frac{1}{2} n_{a} n_{b}} \frac{\left|\sigma_{b} \tau^{\frac{1}{2} n_{b}}-\sigma_{a} \tau^{\frac{1}{2} n_{a}}\right|}{1-\sigma_{a} \sigma_{b} \tau^{\frac{1}{2}\left(n_{a}+n_{b}\right)}} .
$$

Proof. We rewrite the limit as

$$
\lim _{w \rightarrow 1}\left(\mathfrak{h}_{1} \mathfrak{h}_{2}\right)\left(\sigma_{a} \tau^{-\frac{1}{2} n_{a}} w, \sigma_{b} \tau^{-\frac{1}{2} n_{b}} ; n_{a}, n_{b}\right) .
$$

A simple computation (see the proof of Lemma 3.5 for a similar one) leads to

$$
\left(\mathfrak{h}_{1} \mathfrak{h}_{2}\right)\left(\sigma_{a} \tau^{-\frac{1}{2} n_{a}} w, \sigma_{b} \tau^{-\frac{1}{2} n_{b}} ; n_{a}, n_{b}\right)=\prod_{\ell=1}^{n_{a}} \frac{1-\tau^{\ell-\frac{1}{2} n_{a}-\frac{1}{2} n_{b}} \bar{w}}{1-\tau^{\ell-\frac{1}{2} n_{a}+\frac{1}{2} n_{b}} \bar{w}}
$$


where $\bar{w}=\sigma_{a} \sigma_{b} w$. This product equals

$$
\begin{aligned}
\prod_{\ell=1}^{n_{a}} \frac{1-\tau^{1-\ell+\frac{1}{2} n_{a}-\frac{1}{2} n_{b}} \bar{w}}{1-\tau^{\ell-\frac{1}{2} n_{a}+\frac{1}{2} n_{b}} \bar{w}} & =\prod_{\ell=1}^{n_{a}} \frac{\bar{w}-\tau^{\ell-1-\frac{1}{2} n_{a}+\frac{1}{2} n_{b}}}{1-\tau^{\ell-\frac{1}{2} n_{a}+\frac{1}{2} n_{b}} \bar{w}}\left(-\tau^{1-\ell+\frac{1}{2} n_{a}-\frac{1}{2} n_{b}}\right) \\
& =(-1)^{n_{a}} \tau^{\frac{1}{2} n_{a}-\frac{1}{2} n_{a} n_{b}} \frac{\bar{w}-\tau^{\frac{1}{2} n_{b}-\frac{1}{2} n_{a}}}{1-\tau^{\frac{1}{2} n_{a}+\frac{1}{2} n_{b}} \bar{w}} \frac{\prod_{\ell=2}^{n_{a}}\left(\bar{w}-\tau^{\ell-1-\frac{1}{2} n_{a}+\frac{1}{2} n_{b}}\right)}{\prod_{\ell=1}^{n_{a}-1}\left(1-\tau^{\ell-\frac{1}{2} n_{a}+\frac{1}{2} n_{b}} \bar{w}\right)}
\end{aligned}
$$

The last ratio equals $\prod_{\ell=1}^{n_{a}-1} \frac{\sigma_{a} \sigma_{b} w-\tau^{\ell-\frac{1}{2} n_{a}+\frac{1}{2} n_{b}}}{1-\tau^{\ell-\frac{1}{2} n_{a}+\frac{1}{2} n_{b}} \sigma_{a} \sigma_{b} w}$, and each factor in this product tends to $\sigma_{a} \sigma_{b}$ as $w \rightarrow 1$ unless $\ell-\frac{1}{2} n_{a}+\frac{1}{2} n_{b}=0$, in which case it tends to -1 . Therefore the whole ratio goes to $\left(\sigma_{a} \sigma_{b}\right)^{n_{a}-2}(-1)^{\mathbf{1}_{n_{a}-n_{b} \in 2 \mathbb{Z}>0}}\left(\sigma_{a} \sigma_{b}\right)^{1-\mathbf{1}_{n_{a}-n_{b} \in 2 \mathbb{Z}}>0}=\left(\sigma_{a} \sigma_{b}\right)^{n_{a}-1}\left(-\sigma_{a} \sigma_{b}\right)^{\mathbf{1}_{n_{a}-n_{b} \in 2 \mathbb{Z}}>0}$, and thus the limit in (3.6) equals

$$
\begin{aligned}
& -\left(-\sigma_{a} \sigma_{b}\right)^{n_{a}+\mathbf{1}_{n_{a}-n_{b} \in 2 \mathbb{Z}_{>0}}-1} \tau^{\frac{1}{2} n_{a}-\frac{1}{2} n_{a} n_{b}} \frac{\sigma_{a} \sigma_{b}-\tau^{\frac{1}{2} n_{b}-\frac{1}{2} n_{a}}}{1-\tau^{\frac{1}{2} n_{a}+\frac{1}{2} n_{b}} \sigma_{a} \sigma_{b}} \\
& = \begin{cases}(-1)^{n_{a} \wedge n_{b}} \tau^{-\frac{1}{2} n_{a} n_{b}} \frac{\left|\tau^{\frac{1}{2} n_{b}}-\tau^{\frac{1}{2} n_{a}}\right|}{1-\tau^{\frac{1}{2} n_{a}+\frac{1}{2} n_{b}}} & \text { if } \sigma_{a} \sigma_{b}=1 \\
\tau^{-\frac{1}{2} n_{a} n_{b}} \frac{\tau^{\frac{1}{2} n_{b}+\tau^{\frac{1}{2} n_{a}}}}{1+\tau^{\frac{1}{2} n_{a}+\frac{1}{2} n_{b}}} & \text { if } \sigma_{a} \sigma_{b}=-1,\end{cases}
\end{aligned}
$$

which gives the claimed formula.

3.2. Contour deformation and computation of the limit. As we mentioned, we will move the contours one by one, starting from $w_{k}$, then $w_{k-1}$, and so on. From the above discussion, there are two types of poles which are crossed. First, as we expand the $w_{a}$ contour we will cross singularities at $w_{a}= \pm \tau^{-n_{a} / 2}$ as in (c) above. We will refer to these as unpaired poles. The $n$ variables associated to these poles will be called unpaired variables, and will be denoted with a superscript $\mathrm{u}$ (note that unpaired poles have no $w$ variables associated to them, since a residue has been computed).

The second type of pole appears at $w_{a}=w_{b}^{-1} \tau^{-n_{b}}$ (for $a<b$ ) when $w_{b} \in C_{0}^{n_{b}}$ and $n_{a}=n_{b}$. We refer to these as paired poles. To each paired pole corresponds a pair of $w$ variables and a pair of $n$ variables: $w_{b}$, which is integrated over $C_{0}^{n_{b}}$, and $w_{a}$, which is set to $w_{a}=w_{b}^{-1} \tau^{-n_{b}}$, and similarly $n_{b}$ and $n_{a}$, which are set to be equal. We call these paired variables, and denote them with a superscript $\mathrm{p}$.

Finally, in addition to the paired and unpaired variables, there are free variables, denoted with a superscript $\mathrm{f}$, corresponding to the $w$ variables (and associated $n$ variables) which, after the deformation, end up integrated on a $C_{0}^{n}$ contour (note that these have no paired variable associated to them).

The result of deforming all the $w_{a}$ contours involves a sum over all possible ways in which the $k$ original variables can be grouped into $k_{\mathrm{u}}$ unpaired variables, $k_{\mathrm{p}}$ pairs of paired variables, and $k_{\mathrm{f}}$ free variables, which of course means that $k_{\mathrm{u}}+2 k_{\mathrm{p}}+k_{\mathrm{f}}=k$. Given such a grouping, the original $w_{a}$ 's and $n_{a}$ 's will be split into strings of variables: $\vec{n}^{\mathrm{u}}$ for the unpaired ones (of size $k_{\mathrm{u}}$ ), $\vec{n}^{\mathrm{p}}$ and $\vec{w}^{\mathrm{p}}$ for the paired ones (of size $k_{\mathrm{p}}$ ), and $\vec{n}^{\mathrm{f}}$ and $\vec{w}^{\mathrm{f}}$ for the free ones (of size $k_{\mathrm{f}}$ ). It is important to note that in the case of the paired variables, the variables forming these strings will appear twice in the formulas that will follow, once associated to $\vec{w}^{\mathrm{p}}$ and once associated to the "paired" string $\vec{w}^{-\mathrm{p}}$ defined by

$$
w_{a}^{-\mathrm{p}}=\tau^{-n_{a}^{\mathrm{p}}} / w_{a}^{\mathrm{p}} \quad \text { for } a=1, \ldots, k_{\mathrm{p}} .
$$

These variables should be regarded as "virtual", in the sense that they are not being directly summed or integrated, but are instead defined directly in terms of other variables. On the other hand, in the case of the unpaired variables there is an additional string $\vec{\sigma}$ of \pm 1 variables, which will indicate whether the unpaired pole is evaluated at $\tau^{-n_{a} / 2}$ or $-\tau^{-n_{a} / 2}$. 
It will be convenient to introduce another string of virtual variables $\vec{w}^{\mathrm{u}}$, defined as follows:

$$
w_{a}^{\mathrm{u}}=\sigma_{a} \tau^{-\frac{1}{2} n_{a}^{\mathrm{u}}} \quad \text { for } a=1, \ldots, k_{\mathrm{u}} .
$$

Before writing down the result of the contour deformation we need to introduce some additional notation which will help us express some products arising from the pairing structure which emerges from the contour deformation. Assume we are given a collection of pairs of strings of unpaired variables $\left(\vec{w}^{\mathrm{u}}, \vec{n}^{\mathrm{u}}\right)$, paired variables $\left(\vec{w}^{\mathrm{p}}, \vec{n}^{\mathrm{p}}\right)$ and free variables $\left(\vec{w}^{\mathrm{f}}, \vec{n}^{\mathrm{f}}\right)$. Given a function $g$ of a single $w$ and a single $n$ we define

$$
\mathcal{P}^{\mathrm{u}, \mathrm{p}, \mathrm{f}} g\left(\vec{w}^{\mathrm{u}}, \vec{w}^{\mathrm{p}}, \vec{w}^{\mathrm{f}} ; \vec{n}^{\mathrm{u}}, \vec{n}^{\mathrm{p}}, \vec{n}^{\mathrm{f}}\right)=\prod_{a=1}^{k_{\mathrm{u}}} g\left(w_{a}^{\mathrm{u}}, n_{a}^{\mathrm{u}}\right) \prod_{a=1}^{k_{\mathrm{p}}} g\left(w_{a}^{\mathrm{p}}, n_{a}^{\mathrm{p}}\right) g\left(w_{a}^{-\mathrm{p}}, n_{a}^{\mathrm{p}}\right) \prod_{a=1}^{k_{\mathrm{f}}} g\left(w_{a}^{\mathrm{f}}, n_{a}^{\mathrm{f}}\right) .
$$

That is, we multiply over all possible variables, accounting for the extra set of paired ones. Similarly, given a function $h$ of a pair $w_{1}, w_{2}$ and a pair $n_{1}, n_{2}$ we define

$$
\begin{aligned}
\mathcal{P}^{\mathrm{u}, \mathrm{p}, \mathrm{f}}\left(\vec{w}^{\mathrm{u}}, \vec{w}^{\mathrm{p}}, \vec{w}^{\mathrm{f}} ; \vec{n}^{\mathrm{u}}, \vec{n}^{\mathrm{p}}, \vec{n}^{\mathrm{f}}\right)=\prod_{1 \leq a<b \leq k_{\mathrm{u}}} h\left(w_{a}^{\mathrm{u}}, w_{b}^{\mathrm{u}} ; n_{a}^{\mathrm{u}}, n_{b}^{\mathrm{u}}\right) \prod_{1 \leq a<b \leq k_{\mathrm{f}}} h\left(w_{a}^{\mathrm{f}}, w_{b}^{\mathrm{f}} ; n_{a}^{\mathrm{f}}, n_{b}^{\mathrm{f}}\right) \\
\quad \times \prod_{1 \leq a<b \leq k_{\mathrm{p}}} h\left(w_{a}^{\mathrm{p}}, w_{b}^{\mathrm{p}} ; n_{a}^{\mathrm{p}}, n_{b}^{\mathrm{p}}\right) h\left(w_{a}^{\mathrm{p}}, w_{b}^{-\mathrm{p}} ; n_{a}^{\mathrm{p}}, n_{b}^{\mathrm{p}}\right) h\left(w_{a}^{-\mathrm{p}}, w_{b}^{\mathrm{p}} ; n_{a}^{\mathrm{p}}, n_{b}^{\mathrm{p}}\right) h\left(w_{a}^{-\mathrm{p}}, w_{b}^{-\mathrm{p}} ; n_{a}^{\mathrm{p}}, n_{b}^{\mathrm{p}}\right) \\
\quad \times \prod_{a \leq k_{\mathrm{u}}, b \leq k_{\mathrm{p}}} h\left(w_{a}^{\mathrm{u}}, w_{b}^{\mathrm{p}} ; n_{a}^{\mathrm{u}}, n_{b}^{\mathrm{p}}\right) h\left(w_{a}^{\mathrm{u}}, w_{b}^{-\mathrm{p}} ; n_{a}^{\mathrm{u}}, n_{b}^{\mathrm{p}}\right) \prod_{a \leq k_{\mathrm{u}}, b \leq k_{\mathrm{f}}} h\left(w_{a}^{\mathrm{u}}, w_{b}^{\mathrm{f}} ; n_{a}^{\mathrm{u}}, n_{b}^{\mathrm{f}}\right) \\
\quad \times \prod_{a \leq k_{\mathrm{p}}, b \leq k_{\mathrm{f}}} h\left(w_{a}^{\mathrm{p}}, w_{b}^{\mathrm{f}} ; n_{a}^{\mathrm{p}}, n_{b}^{\mathrm{f}}\right) h\left(w_{a}^{-\mathrm{p}}, w_{b}^{\mathrm{f}} ; n_{a}^{\mathrm{p}}, n_{b}^{\mathrm{f}}\right) .
\end{aligned}
$$

Here we multiply over all possible pairs of different variables, accounting again for the extra set of paired variables, but omitting factors with variables from the same pair (that is, omitting factors of the form $\left.h\left(w_{a}^{\mathrm{p}}, w_{a}^{-\mathrm{p}} ; n_{a}^{\mathrm{p}}, n_{a}^{\mathrm{p}}\right)\right)$.

We extend the notations (3.9) and (3.10) as follows: if any group of variables is omitted from the superscript (and the argument), it simply means that those variables are omitted from the products. Thus, for example,

$$
\mathcal{P}^{\mathrm{u}} g\left(\vec{w}^{\mathrm{u}} ; \vec{n}^{\mathrm{u}}\right)=\prod_{a=1}^{k_{\mathrm{u}}} g\left(w_{a}^{\mathrm{u}}, n_{a}^{\mathrm{u}}\right)
$$

while

$$
\begin{aligned}
\mathcal{P}^{\mathrm{u}, \mathrm{p}} h\left(\vec{w}^{\mathrm{u}}, \vec{w}^{\mathrm{p}} ; \vec{n}^{\mathrm{u}}, \vec{n}^{\mathrm{p}}\right) & \\
\quad & =\prod_{1 \leq a<b \leq k_{\mathrm{u}}} h\left(w_{a}^{\mathrm{u}}, w_{b}^{\mathrm{u}} ; n_{a}^{\mathrm{u}}, n_{b}^{\mathrm{u}}\right) \prod_{a \leq k_{\mathrm{u}}, b \leq k_{\mathrm{p}}} h\left(w_{a}^{\mathrm{u}}, w_{b}^{\mathrm{p}} ; n_{a}^{\mathrm{u}}, n_{b}^{\mathrm{p}}\right) h\left(w_{a}^{\mathrm{u}}, w_{b}^{-\mathrm{p}} ; n_{a}^{\mathrm{u}}, n_{b}^{\mathrm{p}}\right) \\
\quad \times & \prod_{1 \leq a<b \leq k_{\mathrm{p}}} h\left(w_{a}^{\mathrm{p}}, w_{b}^{\mathrm{p}} ; n_{a}^{\mathrm{p}}, n_{b}^{\mathrm{p}}\right) h\left(w_{a}^{\mathrm{p}}, w_{b}^{-\mathrm{p}} ; n_{a}^{\mathrm{p}}, n_{b}^{\mathrm{p}}\right) h\left(w_{a}^{-\mathrm{p}}, w_{b}^{\mathrm{p}} ; n_{a}^{\mathrm{p}}, n_{b}^{\mathrm{p}}\right) h\left(w_{a}^{-\mathrm{p}}, w_{b}^{-\mathrm{p}} ; n_{a}^{\mathrm{p}}, n_{b}^{\mathrm{p}}\right) .
\end{aligned}
$$

We will slightly abuse this notation by omitting the superscript in $\mathcal{P}$ (which in any case is implicit in the superscripts of the argument). For convenience we will also write

$$
\mathcal{P h}(\cdot ; \cdot)=\mathcal{P} \mathfrak{h}_{1}(\cdot ; \cdot) \mathcal{P h} \mathfrak{h}_{2}(\cdot ; \cdot) \text {. }
$$

Finally, we introduce the index set

$$
\begin{aligned}
\Lambda_{k_{1}, k_{2}, k_{3}}^{m}=\left\{\left(\vec{\sigma}, \vec{n}^{1}, \vec{n}^{2}, \vec{n}^{3}\right) \in\{-1,1\}^{k_{1}} \times \mathbb{Z}_{\geq 1}^{k_{1}} \times \mathbb{Z}_{\geq_{1}}^{k_{2}} \times \mathbb{Z}_{\geq 1}^{k_{3}}:\right. & \\
& \left.\sum_{a=1}^{k_{1}} n_{a}^{1}+2 \sum_{a=1}^{k_{2}} n_{a}^{2}+\sum_{a=1}^{k_{3}} n_{a}^{3}=m, \sigma_{a} n_{a}^{1} \neq \sigma_{b} n_{b}^{1} \forall 1 \leq a<b \leq k_{1}\right\}
\end{aligned}
$$


and the notation $C_{0}^{\vec{n}}=C_{0}^{n_{1}} \times \cdots \times C_{0}^{n_{k}}$ for $\vec{n}$ of size $k$.

We are ready now to state the result of the complete contour deformation.

Proposition 3.2. For every $k, m \in \mathbb{Z}_{\geq 0}$ with $k \leq m$ we have

$$
\widetilde{\nu}_{k, m}^{\mathrm{h}-\mathrm{fl}}(t, 2 x)=\sum_{\substack{k_{\mathrm{u}}, k_{\mathrm{p}}, k_{\mathrm{f}} \geq 0 \\ k_{\mathrm{u}}+2 k_{\mathrm{p}}+k_{\mathrm{f}}=k}} \frac{1}{k_{\mathrm{u}} ! 2^{k_{\mathrm{p}}} k_{\mathrm{p}} ! k_{\mathrm{f}} !} \mathfrak{Z}_{m}^{\mathrm{h}-\mathrm{fl}}\left(k_{\mathrm{u}}, k_{\mathrm{p}}, k_{\mathrm{f}}\right),
$$

where $\widetilde{\nu}_{k, m}^{\mathrm{h}-\mathrm{fl}}$ was defined in (2.26) and

$$
\begin{aligned}
\mathfrak{Z}_{m}^{\mathrm{h}-\mathrm{fl}}\left(k_{\mathrm{u}}, k_{\mathrm{p}}, k_{\mathrm{f}}\right)= & \sum_{\left(\vec{\sigma}, \vec{n}^{\mathrm{u}}, \vec{n}^{\mathrm{p}}, \vec{n}^{\mathrm{f}}\right) \in \Lambda_{k_{\mathrm{u}}, k_{\mathrm{p}}, k_{\mathrm{f}}}} \frac{1}{(2 \pi \mathrm{i})^{k_{\mathrm{p}}+k_{\mathrm{f}}}} \int_{C_{0}^{\vec{n} \mathrm{p}} \times C_{0}^{\vec{n}^{\mathrm{f}}}} d \vec{w}^{\mathrm{p}} d \vec{w}^{\mathrm{f}} \mathcal{P} \mathfrak{f}_{1}\left(\vec{w}^{\mathrm{u}}, \vec{w}^{\mathrm{p}}, \vec{w}^{\mathrm{f}} ; \vec{n}^{\mathrm{u}}, \vec{n}^{\mathrm{p}}, \vec{n}^{\mathrm{f}}\right) \\
& \times \mathcal{P} \mathfrak{f}_{2}\left(\vec{w}^{\mathrm{f}} ; \vec{n}^{\mathrm{f}}\right) \mathcal{P} \mathfrak{g}_{\mathrm{u}}\left(\vec{w}^{\mathrm{u}} ; \vec{n}^{\mathrm{u}}\right) \mathcal{P} \mathfrak{g}_{\mathrm{p}}\left(\vec{w}^{\mathrm{p}}, \vec{w}^{\mathrm{f}} ; \vec{n}^{\mathrm{p}}, \vec{n}^{\mathrm{f}}\right) \mathcal{P h}\left(\vec{w}^{\mathrm{u}}, \vec{w}^{\mathrm{p}}, \vec{w}^{\mathrm{f}} ; \vec{n}^{\mathrm{u}}, \vec{n}^{\mathrm{p}}, \vec{n}^{\mathrm{f}}\right) \\
& \times \prod_{a=1}^{k_{\mathrm{u}}} \frac{1}{2} w_{a}^{\mathrm{u}} \prod_{a=1}^{k_{\mathrm{p}}}(-1)^{n_{a}^{\mathrm{p}}} \tau^{-n_{a}^{\mathrm{p}}\left(n_{a}^{\mathrm{p}}+1\right) / 2}\left(1-\tau^{n_{a}^{\mathrm{p}}}\right) w_{a}^{-\mathrm{p}} \mathfrak{h}_{2}\left(w_{a}^{\mathrm{p}}, w_{a}^{-\mathrm{p}} ; n_{a}^{\mathrm{p}}, n_{a}^{\mathrm{p}}\right) .
\end{aligned}
$$

The proposition follows from a slightly more general result which we prove next. Define the annulus $R_{0}^{n}=\left\{w \in \mathbb{C}: \tau^{-\eta} \leq|w| \leq \tau^{-n / 2-\eta}\right\}$ and the set $D=\prod_{n \geq 1} R_{0}^{n} \times\{n\}$. We assume as given functions $\phi: D \longrightarrow \mathbb{C}$ and $\psi: D \times D \longrightarrow \mathbb{C}$ such that $\phi(w, n)$ is analytic in $w \in R_{0}^{n}$ and $\psi\left(w_{1}, w_{2} ; n_{1}, n_{2}\right)$ is analytic in each variable $w_{1} \in R_{0}^{n_{1}}$ and $w_{2} \in R_{0}^{n_{2}}$ (we are slightly abusing notation here to write $\psi\left(w_{1}, w_{2} ; n_{1}, n_{2}\right)$ instead of $\left.\psi\left(\left(w_{1}, n_{1}\right),\left(w_{2}, n_{2}\right)\right)\right)$.

Proposition 3.3. Fix $k, m \in \mathbb{Z}_{\geq 1}$ with $k \leq m$ and functions as $\phi$ and $\psi$ as above. Then

$$
\begin{aligned}
& \sum_{\substack{n_{1}, \ldots, n_{k} \geq 1 \\
n_{1}+\cdots+n_{k}=m}} \frac{1}{(2 \pi \mathrm{i})^{k}} \int_{C_{0, \tau^{-\eta}}^{k}} d \vec{w} \prod_{a} \phi\left(w_{a}, n_{a}\right) \mathfrak{g}_{\mathrm{p}}\left(w_{a}, n_{a}\right) \prod_{a<b} \psi\left(w_{a}, w_{b} ; n_{a}, n_{b}\right) \mathfrak{h}_{1}\left(w_{a}, w_{b} ; n_{a}, n_{b}\right) \\
&=\sum_{\substack{k_{\mathrm{u}}, k_{\mathrm{p}}, k_{\mathrm{f}} \geq 0 \\
k_{\mathrm{u}}+2 k_{\mathrm{p}}+k_{\mathrm{f}}=k}} \frac{k !}{k_{\mathrm{u}} ! 2^{k_{\mathrm{p}}} k_{\mathrm{p}} ! k_{\mathrm{f}} !} \sum_{\left(\vec{\sigma}, \vec{n}^{\mathrm{u}}, \vec{n}^{\mathrm{p}}, \vec{n}^{\mathrm{f}}\right) \in \Lambda_{k_{\mathrm{u}}, k_{\mathrm{p}}, k_{\mathrm{f}}}^{m}} \frac{1}{(2 \pi \mathrm{i})^{k_{\mathrm{p}}+k_{\mathrm{f}}}} \int_{C_{0}^{\vec{n}^{\mathrm{p}}}} d \vec{w}^{\mathrm{p}} \int_{C_{0}^{\vec{n}^{\mathrm{f}}}} d \vec{w}^{\mathrm{f}} \\
& \times \mathcal{P} \phi\left(\vec{w}^{\mathrm{u}}, \vec{w}^{\mathrm{p}}, \vec{w}^{\mathrm{f}} ; \vec{n}^{\mathrm{u}}, \vec{n}^{\mathrm{p}}, \vec{n}^{\mathrm{f}}\right) \mathcal{P} \psi\left(\vec{w}^{\mathrm{u}}, \vec{w}^{\mathrm{p}}, \vec{w}^{\mathrm{f}} ; \vec{n}^{\mathrm{u}}, \vec{n}^{\mathrm{p}}, \vec{n}^{\mathrm{f}}\right) \\
& \times \mathcal{P} \mathfrak{g}_{\mathrm{u}}\left(\vec{w}^{\mathrm{u}} ; \vec{n}^{\mathrm{u}}\right) \mathcal{P} \mathfrak{g}_{\mathrm{p}}\left(\vec{w}^{\mathrm{p}}, \vec{w}^{\mathrm{f}} ; \vec{n}^{\mathrm{p}}, \vec{n}^{\mathrm{f}}\right) \mathcal{P} \mathfrak{h}_{1}\left(\vec{w}^{\mathrm{u}}, \vec{w}^{\mathrm{p}}, \vec{w}^{\mathrm{f}} ; \vec{n}^{\mathrm{u}}, \vec{n}^{\mathrm{p}}, \vec{n}^{\mathrm{f}}\right) \\
& \times \prod_{a=1}^{k_{\mathrm{u}}} \frac{1}{2} w_{a}^{\mathrm{u}} \prod_{a=1}^{k_{\mathrm{p}}}(-1)^{n_{a}^{\mathrm{p}}} \tau^{-n_{a}^{\mathrm{p}}\left(n_{a}^{\mathrm{p}}+1\right) / 2}\left(1-\tau^{n_{a}^{\mathrm{p}}}\right) w_{a}^{-\mathrm{p}} \psi\left(w_{a}^{\mathrm{p}}, w_{a}^{-\mathrm{p}} ; n_{a}^{\mathrm{p}}, n_{a}^{\mathrm{p}}\right) .
\end{aligned}
$$

Proof. The proof is by induction in $k$ (for fixed $m$ ). First we check the case $k=1$. Note that $\mathcal{P h}_{1}(\vec{w} ; \vec{n})=1$ in this case (because the product is empty), and thus the only poles crossed as we deform are the ones coming from $\mathfrak{g}_{\mathrm{p}}\left(w_{1}, n_{1}\right)$ as in (c) in page 11 . On the other hand, the condition $k_{\mathrm{u}}+2 k_{\mathrm{p}}+k_{\mathrm{f}}=1$ implies that the sum on the right hand side has two terms: one with $k_{\mathrm{u}}=1$ and the other two set to 0 , and one with $k_{\mathrm{u}}=1$ and the other two set to 0 . Since $\phi\left(w_{1}, n_{1}\right)$ is analytic on the region of interest, one checks directly that the first term corresponds to the computation of the residues at the two poles (keeping in mind that the poles are passed from the outside, so we get an additional minus sign), while the second term corresponds to the integral on the deformed contour.

We assume now that the formula holds for a given $k$ and rewrite the integrand in the case $k+1$, for fixed $w_{k+1} \in C_{0, \tau^{-\eta}}$, as

$$
\left(\phi \mathfrak{g}_{\mathrm{p}}\right)\left(w_{k+1}, n_{k+1}\right) \prod_{a=1}^{k}\left(\tilde{\phi} \mathfrak{g}_{\mathrm{p}}\right)\left(w_{a}, n_{a}\right) \prod_{1 \leq a<b \leq k}\left(\psi \mathfrak{h}_{1}\right)\left(w_{a}, w_{b} ; n_{a}, n_{b}\right)\left(w_{a}, w_{b} ; n_{a}, n_{b}\right)
$$


with

$$
\tilde{\phi}\left(w_{a}, n_{a}\right)=\phi\left(w_{a}, n_{a}\right)\left(\psi \mathfrak{h}_{1}\right)\left(w_{a}, w_{k+1} ; n_{a}, n_{k+1}\right) .
$$

By (d.i) in page 11 and the hypotheses on $\phi$ and $\psi, \tilde{\phi}(\cdot, n)$ is analytic in the deformation region and thus, using the inductive hypothesis, the integral equals

$$
\begin{aligned}
& \sum_{n_{k+1}=1}^{m-k} \frac{1}{2 \pi \mathrm{i}} \int_{C_{0, \tau}-\eta} d w_{k+1} \mathfrak{g}_{\mathrm{p}}\left(w_{k+1}, n_{k+1}\right) \phi\left(w_{k+1}, n_{k+1}\right) \sum_{\substack{k_{\mathrm{u}}, k_{\mathrm{p}}, k_{\mathrm{f}} \geq 0 \\
k_{\mathrm{u}}+k_{\mathrm{p}}+k_{\mathrm{f}}=k}} \frac{k !}{k_{\mathrm{u}} ! 2^{k_{\mathrm{p}}} k_{\mathrm{p}} ! k_{\mathrm{f}} !} \\
& \quad \times \sum_{\substack{\left(\vec{\sigma}, \vec{n}^{\mathrm{u}}, \vec{n}^{\mathrm{p}}, \vec{n}^{\mathrm{f}}\right) \in \Lambda_{k_{\mathrm{u}}, k_{\mathrm{p}}, k_{\mathrm{f}}}^{m-n_{k}} \\
\quad}} \frac{1}{(2 \pi \mathrm{i})^{k_{\mathrm{p}}+k_{\mathrm{f}}}} \int_{\widetilde{C}_{0}^{\vec{n}^{\mathrm{p}}} \times C_{0}^{\vec{n}^{\mathrm{f}}}} d \vec{w}^{\mathrm{p}} d \vec{w}^{\mathrm{f}} \mathcal{P} \tilde{\phi}\left(\vec{w}^{\mathrm{u}}, \vec{w}^{\mathrm{p}}, \vec{w}^{\mathrm{f}} ; \vec{n}^{\mathrm{u}}, \vec{n}^{\mathrm{p}}, \vec{n}^{\mathrm{f}}\right) \\
& \quad \times \mathfrak{P}_{\mathrm{u}}\left(\vec{w}^{\mathrm{u}} ; \vec{n}^{\mathrm{u}}\right) \mathcal{P} \mathfrak{g}_{\mathrm{p}}\left(\vec{w}^{\mathrm{p}}, \vec{w}^{\mathrm{f}} ; \vec{n}^{\mathrm{p}}, \vec{n}^{\mathrm{f}}\right) \mathcal{P h}\left(\vec{w}^{\mathrm{u}}, \vec{w}^{\mathrm{p}}, \vec{w}^{\mathrm{f}} ; \vec{n}^{\mathrm{u}}, \vec{n}^{\mathrm{p}}, \vec{n}^{\mathrm{f}}\right) \\
& \quad \times \prod_{a=1}^{k_{\mathrm{u}}} \frac{1}{2} w_{a}^{\mathrm{u}} \prod_{a=1}^{k_{\mathrm{p}}}(-1)^{n_{a}^{\mathrm{p}}} \tau^{-n_{a}^{\mathrm{p}}\left(n_{a}^{\mathrm{p}}+1\right) / 2}\left(1-\tau^{n_{a}^{\mathrm{p}}}\right) w_{a}^{-\mathrm{p}} \psi\left(w_{a}^{\mathrm{p}}, w_{a}^{-\mathrm{p}} ; n_{a}^{\mathrm{p}}, n_{a}^{\mathrm{p}}\right) .
\end{aligned}
$$

Now we have to deform the $w_{k+1}$ contour to $C_{0}^{n_{k+1}}$. We list the singularities that are crossed:

(a) Since $\phi$ is analytic in $w_{k+1}$ in the deformation region, the first line of (3.16) only contributes the poles of $\mathfrak{g}_{\mathrm{p}}\left(w_{k+1}, n_{k+1}\right)$ as specified in (c) in page 11. By (3.1) it is clear that computing the associated residues corresponds to grouping $\left(w_{k+1}, n_{k+1}\right)$ together with the unpaired variables (i.e., turning it into $\left(w_{k_{\mathrm{u}}+1}^{\mathrm{u}}, n_{k_{\mathrm{u}}+1}^{\mathrm{u}}\right)$, keeping (3.8) in mind).

(b) Now we consider the second line of (3.16). The factors involving $\phi$ and $\psi$ are analytic in $w_{k+1}$ in the deformation region. As for the factors involving $\mathfrak{h}_{1}$, by (d) in page 11 , the only ones which contribute poles are those of the form $\mathfrak{h}_{1}\left(w_{a}^{\mathrm{f}}, w_{k+1} ; n_{a}^{\mathrm{f}}, n_{k+1}\right)$ (see in particular (d.ii)). In view of (3.3), this corresponds to removing $\left(w_{a}^{\mathrm{f}}, n_{a}^{\mathrm{f}}\right)$ from the group of free variables and adding it to the paired variables together with $\left(w_{k+1}, n_{k+1}\right)$, which becomes its pair (i.e., the pairs $\left(w_{a}^{\mathrm{f}}, n_{a}^{\mathrm{f}}\right)$ and $\left(w_{k+1}, n_{k+1}\right)$ become $\left(w_{k_{\mathrm{p}}+1}^{\mathrm{p}}, n_{k_{\mathrm{p}}+1}^{\mathrm{p}}\right)$ and $\left(w_{k_{\mathrm{p}}+1}^{-\mathrm{p}}, n_{k_{\mathrm{p}}+1}^{\mathrm{p}}\right)$, keeping $(3.7)$ in mind.

(c) The last two lines of (3.16) do not depend on $w_{k+1}$, and thus contribute no additional poles.

In addition to the residues in (a) and (b) above, which respectively increase $k_{\mathrm{u}}$ and $k_{\mathrm{p}}$ by 1 , we get one more term with $w_{k+1}$ integrated on the contour $C_{0}^{n_{k+1}}$. As in the above cases, this can be thought of as grouping $\left(w_{k+1}, n_{k+1}\right)$ together with the free variables, this time increasing $k_{\mathrm{f}}$ by one.

The above discussion explains the form taken by the integrand on the right hand side of the identity claimed in the proposition (recall that we are passing poles from the outside, accounting for additional minus sign in each case). It only remains to verify that the inductive argument also yields the combinatorial factor $k ! /\left(k_{\mathrm{u}} ! 2^{k_{\mathrm{p}}} k_{\mathrm{p}} ! k_{\mathrm{f}} !\right)$. Consider first those terms in the resulting sum which are grouped into sizes $\left(k_{\mathrm{u}}+1,2 k_{\mathrm{p}}, k_{\mathrm{f}}\right)$ (which satisfy $\left.k_{\mathrm{u}}+1+2 k_{\mathrm{p}}+k_{\mathrm{f}}=k+1\right)$. In terms of our inductive argument, these terms come from deforming the last contour when the first $k$ variables have been grouped into sizes either $\left(k_{\mathrm{u}}, 2 k_{\mathrm{p}}, k_{\mathrm{f}}\right)$ (so the last one is set as unpaired), $\left(k_{\mathrm{u}}+1,2\left(k_{\mathrm{p}}-1\right), k_{\mathrm{f}}+1\right)$ (so the last one is set as paired, which can be done in $k_{\mathrm{f}}+1$ ways), or $\left(k_{\mathrm{u}}+1,2 k_{\mathrm{p}}, k_{\mathrm{f}}-1\right)$ (so the last one is set as free). By the symmetry of the integrand, and since

$$
\begin{array}{r}
\frac{k !}{k_{\mathrm{u}} ! 2^{k_{\mathrm{p}}} k_{\mathrm{p}} ! k_{\mathrm{f}} !}+\frac{k !}{\left(k_{\mathrm{u}}+1\right) ! 2^{k_{\mathrm{p}}-1}\left(k_{\mathrm{p}}-1\right) !\left(k_{\mathrm{f}}+1\right) !}\left(k_{\mathrm{f}}+1\right)+\frac{k !}{\left(k_{\mathrm{u}}+1\right) ! 2^{k_{\mathrm{p}}} k_{\mathrm{p}} !\left(k_{\mathrm{f}}-1\right) !} \\
=\frac{(k+1) !}{\left(k_{\mathrm{u}}+1\right) ! 2^{k_{\mathrm{p}}} k_{\mathrm{p}} ! k_{\mathrm{f}} !},
\end{array}
$$


we obtain the desired combinatorial factor on the resulting formula. The other cases (where $k_{\mathrm{p}}$ or $k_{\mathrm{f}}$ are increased by one) can be treated similarly.

Proof of Proposition 3.2. In Proposition 3.3 set $\phi=\mathfrak{f}_{1} \mathfrak{f}_{2}$ and $\psi_{2}=\mathfrak{h}_{2}$. By (a) and (b) in the list in page 11 as well as the paragraph preceding (3.5), these functions satisfy the hypotheses of the proposition. The result follows from the formula given in that result, noting that, by (3.2) and (3.4), many of the $\mathfrak{f}_{2}$ factors disappear.

We get now to the crucial point. The only factor depending on $x$ in $(3.15)$ is $\mathfrak{f}_{2}\left(w_{a}^{\mathrm{f}}, n_{a}^{\mathrm{f}}\right)$, which corresponds to the free variables. As we mentioned, $\left|\frac{1+\tau^{n_{a}^{\mathrm{f}}} w_{a}^{\mathrm{f}}}{1+w_{a}^{\mathrm{f}}} \tau^{-\frac{1}{2}} n_{a}^{\mathrm{f}}\right|<1$ whenever $\left|w_{a}^{\mathrm{f}}\right|>\tau^{-\frac{1}{2} n_{a}^{\mathrm{f}}}$. Consequently,

$$
\lim _{x \rightarrow \infty} \mathfrak{f}_{2}\left(w_{a}^{\mathrm{f}}, n_{a}^{\mathrm{f}}\right)=0
$$

Now we apply to this to the computation of the limit as $x \rightarrow \infty$ of $\widetilde{\nu}_{k, m}^{\mathrm{h}-\mathrm{fl}}(t, 2 x)$, which in view of Proposition 3.2 amounts to computing the limit of $\mathfrak{Z}_{m}^{\text {h-f }}\left(k_{\mathrm{u}}, k_{\mathrm{p}}\right)$. Since all the sums in (3.15) are finite, while all the integrals are over finite contours and the integrands are continuous along them, we may take the limit inside and conclude that every term with $k_{\mathrm{f}} \geq 1$ vanishes in the limit. Using this, and keeping in mind the notation introduced in (3.9)-(3.14), we deduce the following

Proposition 3.4. For every $m \in \mathbb{Z}_{\geq 0}$ we have

$$
\nu_{k, m}^{\mathrm{flat}}(t):=\lim _{x \rightarrow \infty} \widetilde{\nu}_{k, m}^{\mathrm{h}-\mathrm{fl}}(t, 2 x)=\sum_{\substack{k_{\mathrm{u}}, k_{\mathrm{p}} \geq 0 \\ k_{\mathrm{u}}+2 k_{\mathrm{p}}=k}} \frac{1}{k_{\mathrm{u}} ! 2^{k_{\mathrm{p}}} k_{\mathrm{p}} !} \mathfrak{Z}_{m}^{\text {flat }}\left(k_{\mathrm{u}}, k_{\mathrm{p}}\right)
$$

with

$$
\begin{aligned}
& \mathfrak{Z}_{m}^{\text {flat }}\left(k_{\mathrm{u}}, k_{\mathrm{p}}\right)=\sum_{\left(\vec{\sigma}, \vec{n}^{\mathrm{u}}, \vec{n}^{\mathrm{p}}\right) \in \Lambda_{k_{\mathrm{u}}, k_{\mathrm{p}}}^{m}} \frac{1}{(2 \pi \mathrm{i})^{k_{\mathrm{p}}}} \int_{C_{0}^{\vec{n}^{\mathrm{P}}}} d \vec{w}^{\mathrm{p}} \mathcal{P} \mathfrak{f}_{1}\left(\vec{w}^{\mathrm{u}}, \vec{w}^{\mathrm{p}} ; \vec{n}^{\mathrm{u}}, \vec{n}^{\mathrm{p}}\right) \\
& \times \mathcal{P} \mathfrak{g}_{\mathrm{u}}\left(\vec{w}^{\mathrm{u}} ; \vec{n}^{\mathrm{u}}\right) \mathcal{P} \mathfrak{g}_{\mathrm{p}}\left(\vec{w}^{\mathrm{p}} ; \vec{n}^{\mathrm{p}}\right) \mathcal{P h}\left(\vec{w}^{\mathrm{u}}, \vec{w}^{\mathrm{p}} ; \vec{n}^{\mathrm{u}}, \vec{n}^{\mathrm{p}}\right) \prod_{a=1}^{k_{\mathrm{u}}} \frac{1}{2} w_{a}^{\mathrm{u}} \\
& \times \prod_{a=1}^{k_{\mathrm{p}}}(-1)^{n_{a}^{\mathrm{p}}} \tau^{-n_{a}^{\mathrm{p}}\left(n_{a}^{\mathrm{p}}+1\right) / 2}\left(1-\tau^{n_{a}^{\mathrm{p}}}\right) w_{a}^{-\mathrm{p}} \mathfrak{h}_{2}\left(w_{a}^{\mathrm{p}}, w_{a}^{-\mathrm{p}} ; n_{a}^{\mathrm{p}}, n_{a}^{\mathrm{p}}\right) .
\end{aligned}
$$

3.3. Simplification of the $\mathfrak{h}$ factors. The next step is to observe that there is an important simplification in the factors appearing in $\mathcal{P h}$ (which, we recall, is defined as the product of $\mathcal{P h} \mathfrak{h}_{1}$ and $\mathcal{P h}$ ), due to the emergence of the paired structure. Define

$$
\mathfrak{e}\left(w_{1}, w_{2} ; n_{1}, n_{2}\right)=\frac{\left(1-\tau^{n_{1}} w_{1} w_{2}\right)\left(1-\tau^{n_{2}} w_{1} w_{2}\right)}{\left(1-w_{1} w_{2}\right)\left(1-\tau^{n_{1}+n_{2}} w_{1} w_{2}\right)} .
$$

Lemma 3.5. For any $w_{1}, w_{2} \in \mathbb{C}$ and $n_{1}, n_{2} \in \mathbb{Z}_{\geq 0}$ for which both sides are well defined,

$$
\begin{aligned}
\mathfrak{h}_{1}\left(w_{1}, w_{2} ; n_{1}, n_{2}\right) \mathfrak{h}_{1}\left(\tau^{-n_{1}} / w_{1}, \tau^{-n_{2}} / w_{2} ; n_{1}, n_{2}\right) \\
\quad \times \mathfrak{h}_{2}\left(\tau^{-n_{1}} / w_{1}, w_{2} ; n_{1}, n_{2}\right) \mathfrak{h}_{2}\left(w_{1}, \tau^{-n_{2}} / w_{2} ; n_{1}, n_{2}\right)=\tau^{-n_{1} n_{2}} \mathfrak{e}\left(w_{1}, w_{2} ; n_{1}, n_{2}\right) .
\end{aligned}
$$

Proof. Let $h_{1,2}\left(w_{1}, w_{2} ; n_{1}, n_{2}\right)=\mathfrak{h}_{1}\left(w_{1}, w_{2} ; n_{1}, n_{2}\right) \mathfrak{h}_{2}\left(\tau^{-n_{1}} / w_{1}, w_{2} ; n_{1}, n_{2}\right)$. Noting that the first factor in each of the Pochhammer symbols in $\mathfrak{h}_{1}$ cancel with like factors in $\mathfrak{h}_{2}$ we get

$$
h_{1,2}\left(w_{1}, w_{2} ; n_{1}, n_{2}\right)=\frac{\left(\tau w_{1} w_{2} ; \tau\right)_{\infty}\left(\tau^{1+n_{1}+n_{2}} w_{1} w_{2} ; \tau\right)_{\infty}}{\left(\tau^{1+n_{1}} w_{1} w_{2} ; \tau\right)_{\infty}\left(\tau^{1+n_{2}} w_{1} w_{2} ; \tau\right)_{\infty}}
$$


Then the product $h_{1,2}\left(w_{1}, w_{2} ; n_{1}, n_{2}\right) h_{1,2}\left(\tau^{-n_{1}} / w_{1}, \tau^{-n_{2}} / w_{2} ; n_{1}, n_{2}\right)$, which is what we are trying to compute, equals

$$
\frac{\left(\tau w_{1} w_{2} ; \tau\right)_{\infty}}{\left(\tau^{1+n_{1}} w_{1} w_{2} ; \tau\right)_{\infty}} \frac{\left(\tau /\left(w_{1} w_{2}\right) ; \tau\right)_{\infty}}{\left(\tau^{1-n_{1}} /\left(w_{1} w_{2}\right) ; \tau\right)_{\infty}} \frac{\left(\tau^{1+n_{1}+n_{2}} w_{1} w_{2} ; \tau\right)_{\infty}}{\left(\tau^{1+n_{2}} w_{1} w_{2} ; \tau\right)_{\infty}} \frac{\left(\tau^{1-n_{1}-n_{2}} /\left(w_{1} w_{2}\right) ; \tau\right)_{\infty}}{\left(\tau^{1-n_{2}} /\left(w_{1} w_{2}\right) ; \tau\right)_{\infty}}
$$

Writing $\bar{w}=w_{1} w_{2}$, the product of the first two ratios above equals

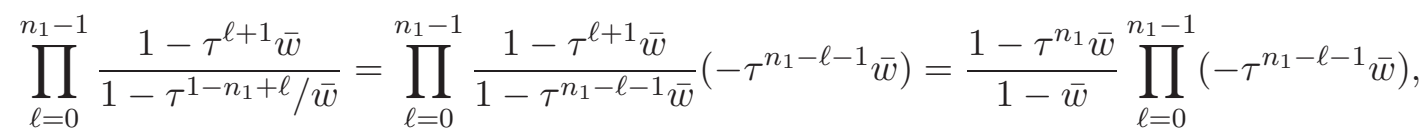

while in a similar way one checks that the product of the last two ratios equals

$$
\prod_{\ell=0}^{n_{1}-1} \frac{1-\tau^{1-n_{1}-n_{2}+\ell} / \bar{w}}{1-\tau^{1+\ell+n_{2}} \bar{w}}=\frac{1-\tau^{n_{2}} \bar{w}}{1-\tau^{n_{1}+n_{2}} \bar{w}} \prod_{\ell=0}^{n_{1}-1} \frac{-1}{\tau^{n_{1}+n_{2}-\ell-1} \bar{w}}
$$

Multiplying out the last two expressions gives the result.

Now note that, in view of (3.7) and (3.8), $\tau^{-n_{a}^{\mathrm{u}}} / w_{a}^{\mathrm{u}}=w_{a}^{\mathrm{u}}, w_{a}^{-\mathrm{p}}=\tau^{-n_{a}^{\mathrm{p}}} / w_{a}^{\mathrm{p}}$ and $\tau^{-n_{a}^{\mathrm{p}}} / w_{a}^{-\mathrm{p}}=$ $w_{a}^{\mathrm{p}}$. Recalling the definition of the product $\mathcal{P} \mathfrak{h}_{1} \mathcal{P h}_{2}$ through (3.12) and pairing the factors coming from the last two products in a suitable way, the lemma implies that

$$
\begin{aligned}
\mathcal{P h}\left(\vec{w}^{\mathrm{u}}, \vec{w}^{\mathrm{p}} ; \vec{n}^{\mathrm{u}}, \vec{n}^{\mathrm{p}}\right)=\mathcal{P h}\left(\vec{w}^{\mathrm{u}} ; \vec{n}^{\mathrm{u}}\right) \prod_{a \leq k_{\mathrm{u}}, b \leq k_{\mathrm{p}}} \tau^{-n_{a}^{\mathrm{u}} n_{b}^{\mathrm{p}} \mathfrak{e}\left(w_{a}^{\mathrm{u}}, w_{b}^{\mathrm{p}} ; n_{a}^{\mathrm{u}}, n_{b}^{\mathrm{p}}\right)} \\
\quad \times \prod_{1 \leq a<b \leq k_{\mathrm{p}}} \tau^{-2 n_{a}^{\mathrm{p}} n_{b}^{\mathrm{p}}} \mathfrak{e}\left(w_{a}^{\mathrm{p}}, w_{b}^{\mathrm{p}} ; n_{a}^{\mathrm{p}}, n_{b}^{\mathrm{p}}\right) \mathfrak{e}\left(w_{a}^{-\mathrm{p}}, w_{b}^{\mathrm{p}} ; n_{a}^{\mathrm{p}}, n_{b}^{\mathrm{p}}\right) .
\end{aligned}
$$

Finally, we introduce the change of variables

$$
w_{a}^{\mathrm{p}}=\tau^{-\frac{1}{2} n_{a}^{\mathrm{p}}} z_{a}^{\mathrm{p}}, \quad w_{a}^{-\mathrm{p}}=\tau^{-\frac{1}{2} n_{a}^{\mathrm{p}}} z_{a}^{-\mathrm{p}}, \quad \text { and } \quad w_{a}^{\mathrm{u}}=\tau^{-\frac{1}{2} n_{a}^{\mathrm{u}}} z_{a}^{\mathrm{u}} .
$$

(Note that, in view of (3.7) and (3.8), $z_{a}^{\mathrm{p}}$ and $z_{a}^{-\mathrm{p}}$ satisfy $z_{a}^{\mathrm{p}}=1 / z_{a}^{-\mathrm{p}}$, while $z_{a}^{\mathrm{u}}=\sigma_{a}$ ). For convenience we also introduce the following notation: given functions $g(z, n)$ and $h\left(z_{1}, z_{2} ; n_{1}, n_{2}\right)$ we write

$$
\tilde{g}(z, n)=g\left(\tau^{-\frac{1}{2} n} z, n\right) \quad \text { and } \quad \tilde{h}\left(z_{1}, z_{2} ; n_{1}, n_{2}\right)=h\left(\tau^{-\frac{1}{2} n_{1}} z_{1}, \tau^{-\frac{1}{2} n_{2}} z_{2} ; n_{1}, n_{2}\right) .
$$

Under the above change of variables the contour $C_{0}^{n_{a}^{\mathrm{p}}}$ is mapped to a circle $C_{0, \tau^{-\eta}}$ of radius $\tau^{-\eta}$ centered at 0. Using this and (3.17) in Proposition 3.4, together with (2.20), (2.25) and (2.26), and multiplying by the factor $\prod_{a} \tau^{-n_{a}^{\mathrm{p}} / 2}$ introduced by the change of variables, we obtain the following:

Theorem 3.6. For every $m \in \mathbb{Z}_{\geq 0}$ we have

$$
\mathbb{E}^{\text {flat }}\left(\tau^{\frac{1}{2} m h(t, 0)}\right)=m_{\tau} ! \sum_{k=0}^{m} \nu_{k, m}^{\text {flat }}(t)
$$

with

$$
\nu_{k, m}^{\text {flat }}(t)=\sum_{\substack{k_{\mathrm{u}}, k_{\mathrm{p}} \geq 0 \\ k_{\mathrm{u}}+2 k_{\mathrm{p}}=k}} \frac{1}{k_{\mathrm{u}} ! 2^{k_{\mathrm{p}}} k_{\mathrm{p}} !} \mathfrak{Z}_{m}^{\text {flat }}\left(k_{\mathrm{u}}, k_{\mathrm{p}}\right)
$$


where

$$
\begin{aligned}
& \mathfrak{Z}_{m}^{\text {flat }}\left(k_{\mathrm{u}}, k_{\mathrm{p}}\right)=\sum_{\left(\vec{\sigma}, \vec{n}^{\mathrm{u}}, \vec{n}^{\mathrm{p}}\right) \in \Lambda_{k_{\mathrm{u}}, k_{\mathrm{p}}}^{m}} \frac{1}{(2 \pi \mathrm{i})^{k_{\mathrm{p}}}} \int_{C_{0,1}^{k_{\mathrm{p}}}} d \vec{z}^{\mathrm{p}} \mathcal{P} \tilde{\mathfrak{f}}_{1}\left(\vec{z}^{\mathrm{u}}, \vec{z}^{\mathrm{p}} ; \vec{n}^{\mathrm{u}}, \vec{n}^{\mathrm{p}}\right) \mathcal{P} \tilde{\mathfrak{g}}_{\mathrm{u}}\left(\vec{z}^{\mathrm{u}} ; \vec{n}^{\mathrm{u}}\right) \mathcal{P} \tilde{\mathfrak{g}}_{\mathrm{p}}\left(\vec{z}^{\mathrm{p}} ; \vec{n}^{\mathrm{p}}\right) \\
& \times \prod_{1 \leq a<b \leq k_{\mathrm{p}}} \tau^{-2 n_{a}^{\mathrm{p}} n_{b}^{\mathrm{p}} \tilde{\mathfrak{e}}}\left(z_{a}^{\mathrm{p}}, z_{b}^{\mathrm{p}} ; n_{a}^{\mathrm{p}}, n_{b}^{\mathrm{p}}\right) \tilde{\mathfrak{e}}\left(z_{a}^{-\mathrm{p}}, z_{b}^{\mathrm{p}} ; n_{a}^{\mathrm{p}}, n_{b}^{\mathrm{p}}\right) \prod_{a \leq k_{\mathrm{u}}, b \leq k_{\mathrm{p}}} \tau^{-n_{a}^{\mathrm{u}} n_{b}^{\mathrm{p}} \tilde{\mathfrak{e}}}\left(z_{a}^{\mathrm{u}}, z_{b}^{\mathrm{p}} ; n_{a}^{\mathrm{u}}, n_{b}^{\mathrm{p}}\right) \\
& \times \mathcal{P} \tilde{\mathfrak{h}}\left(\vec{z}^{\mathrm{u}} ; \vec{n}^{\mathrm{u}}\right) \prod_{a=1}^{k_{\mathrm{u}}} \frac{1}{2} \tau^{-\frac{1}{2} n_{a}^{\mathrm{u}}} z_{a}^{\mathrm{u}} \prod_{a=1}^{k_{\mathrm{p}}}(-1)^{n_{a}^{\mathrm{p}}} \tau^{-\frac{1}{2}\left(n_{a}^{\mathrm{p}}\right)^{2}}\left(\tau^{-n_{a}^{\mathrm{p}}}-1\right) z_{a}^{-\mathrm{p}} \tilde{\mathfrak{h}}_{2}\left(z_{a}^{\mathrm{p}}, z_{a}^{-\mathrm{p}} ; n_{a}^{\mathrm{p}}, n_{a}^{\mathrm{p}}\right),
\end{aligned}
$$

$C_{0,1}$ is a circle of radius 1 centered at the origin, $z_{a}^{-\mathrm{p}}=1 / z_{a}^{\mathrm{p}}$ and $z_{a}^{\mathrm{u}}=\sigma_{a}$.

In particular, this proves Theorem 2.5. Note that in (3.20) we have taken the $z_{a}^{\mathrm{p}}$ contours to lie at circles of radius 1 instead of $\tau^{-\eta}$ as specified by the change of variables (3.18). We may do this at this point because, although both $\tilde{\mathfrak{g}}_{\mathrm{p}}\left(z_{a}^{\mathrm{p}}, n_{a}^{\mathrm{p}}\right)$ and $\tilde{\mathfrak{g}}_{\mathrm{p}}\left(z_{a}^{-\mathrm{p}}, n_{a}\right)$ have simple poles at $z_{a}^{\mathrm{p}}= \pm 1$, the singularities are cancelled by the double zeros of $\tilde{\mathfrak{h}}_{2}\left(z_{a}^{\mathrm{p}}, z_{a}^{-\mathrm{p}} ; n_{a}^{\mathrm{p}}, n_{a}^{\mathrm{p}}\right)$ at $z_{a}^{\mathrm{p}}=1 / z_{a}^{\mathrm{p}}= \pm 1$.

\section{Moment formula: Pfaffian structure}

In this section we will prove Theorem 2.1, starting from the formula given in Theorem 3.6. The structure of the proof is similar to the one provided in [LDC12] for the case of the delta Bose gas.

In order to unveil the Pfaffian structure lying behind the formula in Theorem 3.6 it will be convenient to consider first the case when $k_{\mathrm{u}}$ is even.

4.1. Case $k_{\mathrm{u}}$ even. Define the following variables:

$$
\begin{aligned}
y_{2 a-1} & =z_{a}^{\mathrm{p}}, \quad y_{2 a}=1 / z_{a}^{\mathrm{p}} & & y_{2 k_{\mathrm{p}}+b}=\sigma_{b}, \\
m_{2 a-1} & =m_{2 a}=n_{a}^{\mathrm{p}}, \quad \text { and } & & m_{2 k_{\mathrm{p}}+b}=n_{b}^{\mathrm{u}}
\end{aligned}
$$

for $1 \leq a \leq k_{\mathrm{p}}$ and $1 \leq b \leq k_{\mathrm{u}}$. We will see next how the pairing structure, and in particular the simplification presented in Section 3.3, yields formulas which can be naturally turned into a Pfaffian.

A computation shows that

$$
\prod_{1 \leq a<b \leq k_{\mathrm{p}}} \tilde{\mathfrak{e}}\left(z_{a}^{\mathrm{p}}, z_{b}^{\mathrm{p}} ; n_{a}^{\mathrm{p}}, n_{b}^{\mathrm{p}}\right) \tilde{\mathfrak{e}}\left(z_{a}^{-\mathrm{p}}, z_{b}^{\mathrm{p}} ; n_{a}^{\mathrm{p}}, n_{b}^{\mathrm{p}}\right)=\prod_{1 \leq a<b \leq 2 k_{\mathrm{p}}} \frac{\tau^{\frac{1}{2} m_{b}} y_{b}-\tau^{\frac{1}{2} m_{a}} y_{a}}{\tau^{\frac{1}{2} m_{a}+\frac{1}{2} m_{b}} y_{a} y_{b}-1}
$$

and, similarly,

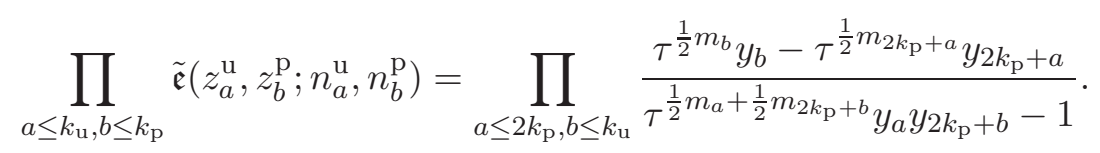

In order to complete the nonlinear Schur Pfaffian $(2.32)$ in $y_{1}, \ldots, y_{2 k_{\mathrm{p}}+k_{\mathrm{u}}}$ we need to multiply by some missing factors, yielding

$$
\begin{aligned}
& \text { (L.H.S. }(4.2)) \times(\text { L.H.S. }(4.3))=\prod_{2 k_{\mathrm{p}}+1 \leq a<b \leq 2 k_{\mathrm{p}}+k_{\mathrm{u}}} \frac{\tau^{\frac{1}{2} m_{a}+\frac{1}{2} m_{b}} y_{a} y_{b}-1}{\tau^{\frac{1}{2} m_{b}} y_{b}-\tau^{\frac{1}{2} m_{a}} y_{a}} \\
& \qquad \times \prod_{a=1}^{k_{\mathrm{p}}} \frac{\tau^{\frac{1}{2} m_{2 a-1}+\frac{1}{2} m_{2 a}} y_{2 a-1} y_{2 a}-1}{\tau^{\frac{1}{2} m_{2 a}} y_{2 a}-\tau^{\frac{1}{2} m_{2 a-1}} y_{2 a-1}} \operatorname{Pf}\left[\frac{\tau^{\frac{1}{2} m_{b}} y_{b}-\tau^{\frac{1}{2} m_{a}} y_{a}}{\tau^{\frac{1}{2}\left(m_{a}+m_{b}\right)} y_{a} y_{b}-1}\right]_{a, b=1}^{2 k_{\mathrm{p}}+k_{\mathrm{u}}} .
\end{aligned}
$$


Now consider the factor $\mathcal{P} \tilde{\mathfrak{h}}\left(\vec{z}^{\mathrm{u}} ; \vec{n}^{\mathrm{u}}\right)$ on the right hand side of $(3.20)$. Since $z_{a}^{\mathrm{u}}=\sigma_{a},(3.5)$ and Lemma 3.1 yield $\tilde{\mathfrak{h}}\left(z_{a}^{\mathrm{u}}, z_{b}^{\mathrm{u}} ; n_{a}^{\mathrm{u}}, n_{b}^{\mathrm{u}}\right)=\left(-\sigma_{a} \sigma_{b}\right)^{n_{a}^{\mathrm{u}} \wedge n_{b}^{\mathrm{u}}} \frac{\left|\sigma_{b} \tau^{\frac{1}{2} n_{b}^{\mathrm{u}}-\sigma_{a} \tau^{\frac{1}{2}} n_{a}^{\mathrm{u}}}\right|}{1-\sigma_{a} \sigma_{b} \tau^{\frac{1}{2}\left(n_{a}^{\mathrm{u}}+n_{b}^{\mathrm{u}}\right)}}$. Using this and (4.4) together with the facts that $\sigma_{a}=y_{2 k_{\mathrm{p}}+a}, \frac{\left|\sigma_{b} \tau^{\frac{1}{2}} n_{b}^{\mathrm{u}}-\sigma_{a} \tau^{\frac{1}{2} n_{a}^{\mathrm{u}}}\right|}{\sigma_{a} \tau^{\frac{1}{2} n_{a}^{\mathrm{u}}}-\sigma_{b} \tau^{\frac{1}{2} n_{b}^{\mathrm{u}}}}=\sigma_{a} \sigma_{b} \operatorname{sgn}\left(\sigma_{b} n_{b}^{\mathrm{u}}-\sigma_{a} n_{a}^{\mathrm{u}}\right)$ and $\frac{\tau^{\frac{1}{2} m_{2 a-1}+\frac{1}{2} m_{2 a} y_{2 a-1} y_{2 a}-1}}{\tau^{\frac{1}{2} m_{2 a}} y_{2 a}-\tau^{\frac{1}{2} m_{2 a-1}} y_{2 a-1}}=\frac{z_{a}^{\mathrm{p}}\left(\tau^{n_{a}^{\mathrm{p}}}-1\right)}{\tau^{\frac{1}{2} n_{a}^{\mathrm{P}}}\left(1-\left(z_{a}^{\mathrm{p}}\right)^{2}\right)}$ yields

$$
\begin{aligned}
\mathcal{P} \tilde{\mathfrak{h}}\left(\vec{z}^{\mathrm{u}} ; \vec{n}^{\mathrm{u}}\right) \times(\text { L.H.S. }(4.2)) \times(\text { L.H.S. }(4.3))=\prod_{1 \leq a<b \leq k_{\mathrm{u}}}\left(-\sigma_{a} \sigma_{b}\right)^{n_{a}^{\mathrm{u}} \wedge n_{b}^{\mathrm{u}}+1} \operatorname{sgn}\left(\sigma_{a} n_{a}^{\mathrm{u}}-\sigma_{b} n_{b}^{\mathrm{u}}\right) \\
\times \prod_{a=1}^{k_{\mathrm{p}}} \frac{z_{a}^{\mathrm{p}}\left(\tau^{n_{a}^{\mathrm{p}}}-1\right)}{\tau^{\frac{1}{2} n_{a}^{\mathrm{p}}}\left(1-\left(z_{a}^{\mathrm{p}}\right)^{2}\right)} \operatorname{Pf}\left[\frac{\tau^{\frac{1}{2} m_{b}} y_{b}-\tau^{\frac{1}{2} m_{a}} y_{a}}{\tau^{\frac{1}{2}\left(m_{a}+m_{b}\right)} y_{a} y_{b}-1}\right]_{a, b=1}^{2 k_{\mathrm{p}}+k_{\mathrm{u}}} \cdot \quad(4.5)
\end{aligned}
$$

It remains to recognize the cross-product on the right hand side of (4.5) as a Pfaffian. As will be clear in the proof of the next lemma, the factors in that product can be thought of as the entries of a degenerate Schur matrix. In the case $\sigma_{a} \equiv 1$, this identity was discovered and checked for small values of $k$ using Mathematica in [LDC12].

Lemma 4.1. For $k \in\{2,4,6, \ldots\}$, positive integers $m_{1}, \ldots, m_{k}$, and $\sigma_{1}, \ldots \sigma_{k} \in\{-1,1\}$,

$\prod_{1 \leq a<b \leq k}\left(-\sigma_{a} \sigma_{b}\right)^{m_{a} \wedge m_{b}+1} \operatorname{sgn}\left(\sigma_{a} m_{a}-\sigma_{b} m_{b}\right)=\operatorname{Pf}\left[\left(-\sigma_{a} \sigma_{b}\right)^{m_{a} \wedge m_{b}} \operatorname{sgn}\left(\sigma_{b} m_{b}-\sigma_{a} m_{a}\right)\right]_{a, b=1}^{k}$

Proof. If any $\sigma_{a} m_{a}=\sigma_{b} m_{b}$, both sides vanish, as $\operatorname{sgn}(0)=0$, so we can assume they are all distinct. Switching $\left(\sigma_{a}, m_{a}\right)$ with a different $\left(\sigma_{b}, m_{b}\right)$ induces a sign change on both sides of the identity, hence by making finitely many such switches, we can assume that $\sigma_{i}=1, i=1, \ldots, n$, and $\sigma_{i}=-1, i=n+1, \ldots, k$. Our matrix then has the block form $\left[\begin{array}{cc}A & D \\ -D^{\top} & B\end{array}\right]$ where $A$ is $k \times k, B$ is $(k-n) \times(k-n)$ and the $k \times(n-k)$ matrix $D$ has all entries $D_{a b}=1$. Since $D$ is rank one, $\operatorname{Pf}\left[\begin{array}{rr}A & D \\ -D^{\top} & B\end{array}\right]=\operatorname{Pf}[A] \operatorname{Pf}[B]$ by (B.5) below. Choose $p(h, m)$ to be a function of $h \in \mathbb{R}, m \in \mathbb{Z}$ satisfying $p(h, m) \rightarrow \infty$ as $h \rightarrow 0$ if $m$ is even, and $p(h, m) \rightarrow 0$ as $h \rightarrow 0$ if $m$ is odd. Let $A_{a, b}(\vec{h}, \vec{m})=\frac{p\left(h_{b}, m_{b}\right)-p\left(h_{a}, m_{a}\right)}{p\left(h_{b}, m_{b}\right)+p\left(h_{a}, m_{a}\right)}$ for $a, b=1, \ldots, n$ and $B_{a, b}(\vec{h}, \vec{m})=\frac{p\left(h_{b}, m_{b}\right)-p\left(h_{a}, m_{a}\right)}{p\left(h_{b}, m_{b}\right)+p\left(h_{a}, m_{a}\right)}$ for $a, b=n+1, \ldots, k$. They have the property that if $h_{a} \rightarrow 0$ in increasing order of $m_{a}$ (i.e. if $m_{b}>m_{a}$, then $h_{a} \rightarrow 0$ followed by $h_{b} \rightarrow 0$ ) for $a, b$ with $\sigma_{a}=\sigma_{b}=1$ (i.e. $1 \leq a, b \leq n$ ) and in decreasing order of $m_{a}$ for $\sigma_{a}=\sigma_{b}=-1$ (i.e. $n+1 \leq a \leq k$ ) then both $A_{a, b}(\vec{h}, \vec{m})$ and $B_{a, b}(\vec{h}, \vec{m})$ converge to $\left(-\sigma_{a} \sigma_{b}\right)^{m_{a} \wedge m_{b}+1} \operatorname{sgn}\left(\sigma_{a} m_{a}-\sigma_{b} m_{b}\right)$. By the Schur Pfaffian formula (2.31), we have $\operatorname{Pf}[A(\vec{h}, \vec{m})] \operatorname{Pf}[B(\vec{h}, \vec{m})]=\prod_{1 \leq a<b \leq n} A_{a, b}(\vec{h}, \vec{m}) \prod_{n+1 \leq a<b \leq k} B_{a, b}(\vec{h}, \vec{m})$. Taking the limit in the given order yields the result.

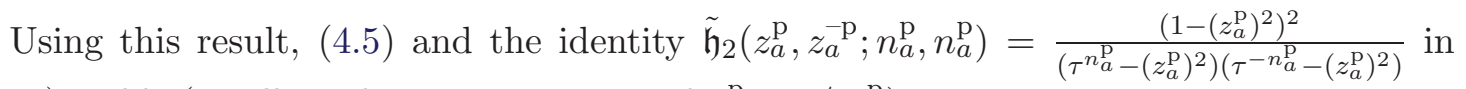
(3.20) yields (recalling that $\sigma_{a}=y_{2 k_{\mathrm{p}}+a}$ and $z_{a}^{\mathrm{p}}=1 / z_{a}^{-\mathrm{p}}$ ) 


$$
\begin{aligned}
& \mathfrak{Z}_{m}^{\text {flat }}\left(k_{\mathrm{u}}, k_{\mathrm{p}}\right)=\sum_{\substack{\vec{\sigma} \in\{-1,1\}^{k_{\mathrm{u}}}, \vec{n}^{\mathrm{u}} \in \mathbb{Z}_{\geq 1}^{k_{\mathrm{u}}}, \vec{z}^{\mathrm{p}} \in \mathbb{Z}_{\geq 1}^{k_{\mathrm{p}}} \\
\sum n_{a}^{\mathrm{u}}+2 \sum n_{a}^{\mathrm{p}}=m}} \frac{1}{(2 \pi \mathrm{i})^{k_{\mathrm{p}}}} \int_{C_{0,1}^{k_{\mathrm{p}}}} d \vec{z}^{\mathrm{p}} \prod_{1 \leq a<b \leq k_{\mathrm{p}}} \tau^{-2 n_{a}^{\mathrm{p}} n_{b}^{\mathrm{p}}} \prod_{a \leq k_{\mathrm{u}}, b \leq k_{\mathrm{p}}} \tau^{-n_{a}^{\mathrm{u}} n_{b}^{\mathrm{p}}} \prod_{1 \leq a<b \leq k_{\mathrm{u}}} \tau^{-\frac{1}{2} n_{a}^{\mathrm{u}} n_{b}^{\mathrm{u}}} \\
& \times \mathcal{P} \tilde{\mathfrak{f}}_{1}\left(\vec{z}^{\mathrm{u}}, \vec{z}^{\mathrm{p}} ; \vec{n}^{\mathrm{u}}, \vec{n}^{\mathrm{p}}\right) \mathcal{P} \tilde{\mathfrak{g}}_{\mathrm{u}}\left(\vec{z}^{\mathrm{u}} ; \vec{n}^{\mathrm{u}}\right) \mathcal{P} \tilde{\mathfrak{g}}_{\mathrm{p}}\left(\vec{z}^{\mathrm{p}} ; \vec{n}^{\mathrm{p}}\right) \prod_{a=1}^{k_{\mathrm{u}}} \frac{1}{2} \tau^{-\frac{1}{2} n_{a}^{\mathrm{u}}} z_{a}^{\mathrm{u}} \\
& \times \prod_{a=1}^{k_{\mathrm{p}}}(-1)^{n_{a}^{\mathrm{p}}} \tau^{-\frac{1}{2} n_{a}^{\mathrm{p}}\left(n_{a}^{\mathrm{p}}+1\right)} \frac{\tau^{-n_{a}^{\mathrm{p}}}\left(1-\left(z_{a}^{\mathrm{p}}\right)^{2}\right)^{2}}{\left(\tau^{n_{a}^{\mathrm{p}}}-\left(z_{a}^{\mathrm{p}}\right)^{2}\right)\left(\tau^{-n_{a}^{\mathrm{p}}}-\left(z_{a}^{\mathrm{p}}\right)^{2}\right)} \frac{\left(1-\tau^{n_{a}^{\mathrm{p}}}\right)^{2}}{\left(\left(z_{a}^{\mathrm{p}}\right)^{2}-1\right)} \\
& \times \operatorname{Pf}\left[\frac{\tau^{\frac{1}{2} m_{b}} y_{b}-\tau^{\frac{1}{2} m_{a}} y_{a}}{\tau^{\frac{1}{2}\left(m_{a}+m_{b}\right)} y_{a} y_{b}-1}\right]_{a, b=1}^{2 k_{\mathrm{p}}+k_{\mathrm{u}}} \operatorname{Pf}\left[\left(-y_{a} y_{b}\right)^{m_{a} \wedge m_{b}} \operatorname{sgn}\left(y_{b} m_{b}-y_{a} m_{a}\right)\right]_{a, b=2 k_{\mathrm{p}}+1}^{2 k_{\mathrm{p}}+k_{\mathrm{u}}} .
\end{aligned}
$$

Note that we have removed the restriction that the $\sigma_{a} n_{a}^{\mathrm{u}}$ 's be different at this point. We may do this thanks to the second Pfaffian on the right hand side. In fact, by Lemma 4.1 this Pfaffian contains a factor $\prod_{a<b} \operatorname{sgn}\left(\sigma_{b} m_{b}-\sigma_{a} m_{a}\right)$, which vanishes if a pair of $\sigma_{a} n_{a}^{\mathrm{u}}$ 's are equal. Now note that

$$
\tilde{\mathfrak{g}}_{\mathrm{p}}(z, n) \tilde{\mathfrak{g}}_{\mathrm{p}}(1 / z, n) \frac{\tau^{-n}\left(1-z^{2}\right)}{\left(\tau^{n}-z^{2}\right)\left(\tau^{-n}-z^{2}\right)}=\frac{\left(-\tau^{-n / 2} z ; \tau\right)_{\infty}\left(-\tau^{-n / 2} / z ; \tau\right)}{\left(-\tau^{n / 2} z ; \tau\right)_{\infty}\left(-\tau^{n / 2} / z ; \tau\right)_{\infty}} \frac{\left(\tau^{1+n} z^{2} ; \tau\right)_{\infty}\left(\tau^{1+n} / z^{2} ; \tau\right)_{\infty}}{\left(\tau z^{2} ; \tau\right)_{\infty}\left(\tau / z^{2} ; \tau\right)_{\infty}} .
$$

With this in mind we introduce the notation

$$
\begin{gathered}
\mathfrak{u}(z, n)=\left(1-\tau^{n}\right) \mathfrak{f}_{1}\left(\tau^{-\frac{1}{2} n} z ; n\right) \frac{\left(-\tau^{-n / 2} z ; \tau\right)_{\infty}\left(\tau^{1+n} z^{2} ; \tau\right)_{\infty}}{\left(-\tau^{n / 2} z ; \tau\right)_{\infty}\left(\tau z^{2} ; \tau\right)_{\infty}}, \\
\mathfrak{u}_{\mathrm{u}}(z, n)=\tau^{-\frac{1}{2} n} z \frac{1-\tau^{n} z^{2}}{1-\tau^{n}}, \quad \mathfrak{u}_{\mathrm{p}}(z, n)=(-1)^{n} \tau^{-\frac{1}{2} n} \frac{1}{z^{2}-1}, \\
\mathfrak{u}_{\mathrm{ap}}(z, n)=(-1)^{n} \tau^{-\frac{1}{2} n} \frac{1+z^{2}}{z^{2}-1},
\end{gathered}
$$

chosen so that

$\left(2^{\text {nd }}\right.$ line $\left.(4.6)\right) \times\left(3^{\text {rd }}\right.$ line $\left.(4.6)\right)=\frac{1}{2^{k_{\mathrm{u}}}} \mathcal{P} \mathfrak{u}\left(\vec{z}^{\mathrm{p}}, \vec{z}^{\mathrm{u}} ; \vec{n}^{\mathrm{p}}, \vec{n}^{\mathrm{u}}\right) \mathcal{P} \mathfrak{u}_{\mathrm{u}}\left(\vec{z}^{\mathrm{u}} ; \vec{n}^{\mathrm{u}}\right) \prod_{a=1}^{k_{\mathrm{p}}} \mathfrak{u}_{\mathrm{p}}\left(z_{a}^{\mathrm{p}}, n_{a}^{\mathrm{p}}\right) \tau^{-\frac{1}{2}\left(n_{a}^{\mathrm{p}}\right)^{2}}$

$\left(\mathfrak{u}_{\text {ap }}\right.$ is a certain antisymmetrization of $\mathfrak{u}_{\mathrm{p}}$, see (4.12), and will be used later on). Of course, this decomposition is not unique, but it will turn out to be convenient below.

Observe that, in view of (4.1), all the $y_{a}$ 's live in $C_{0,1}$. Therefore we may replace the integration in $\vec{z}^{\mathrm{p}}$ over $C_{0,1}^{k_{\mathrm{p}}}$ by an integration in $\vec{y}$ over $C_{0,1}^{2 k_{\mathrm{p}}+k_{\mathrm{u}}}$ by introducing suitable delta functions. We may similarly sum over $m_{1}, \ldots, m_{2 k_{\mathrm{p}}+k_{\mathrm{u}}} \in \mathbb{Z}_{\geq 1}$ and get rid of the sum over $\sigma_{1}, \ldots, \sigma_{k_{\mathrm{u}}} \in\{-1,1\}$. The precise replacement we are going to use, keeping in mind (4.1), can be expressed as follows: for a suitable function $f$,

$$
\begin{aligned}
& \sum_{\substack{\vec{\sigma} \in\{-1,1\}^{k_{\mathrm{u}}}, \vec{n}^{\mathrm{u}} \in \mathbb{Z}_{\mathbb{Z}_{\mathrm{u}}}^{k_{\mathrm{u}}}, \vec{z}^{\mathrm{p}} \in \mathbb{Z}_{\geq 1}^{k_{\mathrm{p}}} \\
\sum n_{a}^{\mathrm{u}}+2 \sum n_{a}^{\mathrm{p}}=m}} \frac{1}{(2 \pi \mathrm{i})^{k_{\mathrm{p}}}} \int_{C_{0,1}^{k_{\mathrm{p}}}} d \vec{z}^{\mathrm{p}} f\left(\vec{z}^{\mathrm{p}} ; \vec{z}^{\mathrm{p}} ; \vec{n}^{\mathrm{p}} ; \vec{n}^{\mathrm{p}} ; \vec{n}^{\mathrm{u}} ; \vec{\sigma}\right) \\
& =\sum_{\substack{m_{1}, \ldots, m_{2 k_{\mathrm{p}}+k_{\mathrm{u}}=1}=1 \\
m_{1}+\cdots+m_{2 k_{\mathrm{p}}+k_{\mathrm{u}}}=m}} \frac{1}{(2 \pi \mathrm{i})^{2 k_{\mathrm{p}}+k_{\mathrm{u}}}} \int_{C_{1}^{2 k_{\mathrm{p}}+k_{\mathrm{u}}}} d \vec{y} \prod_{a=1}^{k_{\mathrm{p}}} \delta_{y_{2 a-1}-\frac{1}{y_{2 a}}} \mathbf{1}_{m_{2 a-1}=m_{2 a}} \prod_{a=2 k_{\mathrm{p}}+a}^{2 k_{\mathrm{p}}+k_{\mathrm{u}}}\left(\delta_{y_{a}-1}+\delta_{y_{a}+1}\right) \\
& \times f\left(y_{1}, y_{3}, \ldots, y_{2 k_{\mathrm{p}}-1} ; y_{2}, y_{4}, \ldots, y_{2 k_{\mathrm{p}}} ; m_{1}, m_{3}, \ldots, m_{2 k_{\mathrm{p}}-1} ;\right. \\
& m_{2}, m_{4}, \ldots, m_{2 k_{\mathrm{p}}} ; m_{2 k_{\mathrm{p}}+1}, \ldots, m_{2 k_{\mathrm{p}}+k_{\mathrm{u}}} ; y_{2 k_{\mathrm{p}}+1}, \ldots, y_{\left.2 k_{\mathrm{p}}+k_{\mathrm{u}}\right) .} \\
& \quad
\end{aligned}
$$


The proof of this (essentially algebraic) identity is straightforward. Here, in view of the fact that we are working with contour integrals in the complex plane, our delta functions implicitly carry a factor of $2 \pi \mathrm{i}$ (which is omitted for notational convenience).

Remark 4.2. The use of delta functions here will should be regarded essentially as a purely notational device which will allow us to express our integral kernels in a more convenient way. In most parts of the argument it will not cause any difficulty, with the exception of the argument following (4.20) which is treated in pages 26-28. In particular, the delta functions will be gone by the time we get to our main result, Theorem 2.1. The reader who feels uncomfortable with this treatment can replace $\delta_{z}$ by a symmetric mollifier $\varphi_{\varepsilon}$ (so that $\varphi_{\varepsilon}(x) \longrightarrow \delta_{x}$ as $\varepsilon \rightarrow 0$ in the sense of distributions), and replace the right hand side of (4.9) by

$$
\begin{aligned}
& \sum_{\substack{m_{1}, \ldots, m_{2 k_{\mathrm{p}}+k_{\mathrm{u}}=1,} \\
m_{1}+\cdots+m_{2 k_{\mathrm{p}}+k_{\mathrm{u}}=}}}^{\infty} \frac{1}{(2 \pi \mathrm{i})^{2 k_{\mathrm{p}}+k_{\mathrm{u}}}} \int_{C_{0,1}^{2 k_{\mathrm{p}}+k_{\mathrm{u}}}} d \vec{y} f(\star) \\
& \times \prod_{a=1}^{k_{\mathrm{p}}} \varphi_{\varepsilon_{a}}\left(y_{2 a-1}-1 / y_{2 a}\right) \mathbf{1}_{m_{2 a-1}=m_{2 a}} \prod_{a=2 k_{\mathrm{p}}+a}^{2 k_{\mathrm{p}}+k_{\mathrm{u}}}\left(\varphi_{\varepsilon_{a}}\left(y_{a}-1\right)+\varphi_{\varepsilon_{a}}\left(y_{a}+1\right)\right),
\end{aligned}
$$

where $f(\star)$ stands for the factor (with the same arguments) appearing on the last two lines of (4.9). This is what we do in pages 26-28.

Using this idea, (4.8), and noting that by the definition (4.1) of the $m_{a}$ 's in terms of the $n_{a}^{\mathrm{u}}$ 's and $n_{a}^{\mathrm{p}}$ 's

$$
\prod_{a=1}^{k_{\mathrm{p}}} \tau^{-\frac{1}{2}\left(n_{a}^{\mathrm{p}}\right)^{2}} \prod_{1 \leq a<b \leq k_{\mathrm{p}}} \tau^{-2 n_{a}^{\mathrm{p}} n_{b}^{\mathrm{p}}} \prod_{a \leq k_{\mathrm{u}}, b \leq k_{\mathrm{p}}} \tau^{-n_{a}^{\mathrm{u}} n_{b}^{\mathrm{p}}} \prod_{1 \leq a<b \leq k_{\mathrm{u}}} \tau^{-\frac{1}{2} n_{a}^{\mathrm{u}} n_{b}^{\mathrm{u}}}=\prod_{1 \leq a<b \leq 2 k_{\mathrm{p}}+k_{\mathrm{u}}} \tau^{-\frac{1}{2} m_{a} m_{b}},
$$

(4.6) becomes $^{1}$

$$
\begin{aligned}
& \mathfrak{Z}_{m}^{\text {flat }}\left(k_{\mathrm{u}}, k_{\mathrm{p}}\right)=\frac{1}{2^{k_{\mathrm{u}}}} \sum_{\substack{m_{1}, \ldots, m_{2 k_{\mathrm{p}}+k_{\mathrm{u}}}=1, m_{1}+\cdots+m_{2 k_{\mathrm{p}}+k_{\mathrm{u}}}=m}}^{\infty} \frac{1}{(2 \pi \mathrm{i})^{2 k_{\mathrm{p}}+k_{\mathrm{u}}}} \int_{C_{0,1}^{2 k_{\mathrm{p}}+k_{\mathrm{u}}}} d \vec{y} \prod_{1 \leq a<b \leq 2 k_{\mathrm{p}}+k_{\mathrm{u}}} \tau^{-\frac{1}{2} m_{a} m_{b}} \\
& \times \prod_{a=1}^{2 k_{\mathrm{p}}+k_{\mathrm{u}}} \mathfrak{u}\left(y_{a}, m_{a}\right) \prod_{a=2 k_{\mathrm{p}}+1}^{2 k_{\mathrm{p}}+k_{\mathrm{u}}} \mathfrak{u}_{\mathrm{u}}\left(y_{a}, m_{a}\right)\left(\delta_{y_{a}-1}+\delta_{y_{a}+1}\right) \prod_{a=1}^{k_{\mathrm{p}}} \mathfrak{u}_{\mathrm{p}}\left(y_{a}, m_{a}\right) \delta_{y_{2 a-1}-\frac{1}{y_{2 a}}} \mathbf{1}_{m_{2 a-1}=m_{2 a}} \\
& \times \operatorname{Pf}\left[\frac{\tau^{\frac{1}{2} m_{b}} y_{b}-\tau^{\frac{1}{2} m_{a}} y_{a}}{\tau^{\frac{1}{2}\left(m_{a}+m_{b}\right)} y_{a} y_{b}-1}\right]_{a, b=1}^{2 k_{\mathrm{p}}+k_{\mathrm{u}}} \operatorname{Pf}\left[\left(-y_{a} y_{b}\right)^{m_{a} \wedge m_{b}} \operatorname{sgn}\left(y_{b} m_{b}-y_{a} m_{a}\right)\right]_{a, b=2 k_{\mathrm{p}}+1}^{2 k_{\mathrm{p}}+k_{\mathrm{u}}} .
\end{aligned}
$$

Now observe that the integration measure $\sum_{m_{1}+\cdots+m_{2 k_{\mathrm{p}}+k_{\mathrm{u}}}=m}^{\infty} \int_{C_{0,1}^{2 k_{\mathrm{p}}+k_{\mathrm{u}}}} d \vec{y}$ is symmetric under exchanging $m_{a}$ 's and $y_{a}$ 's. Let $\pi_{a} \in S_{2 k_{\mathrm{p}}}$ denote the transposition of elements $2 a-1$ and $2 a$ and let $T_{2 k_{\mathrm{p}}}$ be the subgroup of $S_{2 k_{\mathrm{p}}}$ generated by $\left\{\pi_{a}, a=1, \ldots, k_{\mathrm{p}}\right\}$, which has order $2^{k_{\mathrm{p}}}$. Noting every factor in (4.10) is invariant under the action of $T_{2 k_{\mathrm{p}}}$ on the $2 k_{\mathrm{p}}$-tuple $\left(\left(m_{1}, y_{1}\right), \ldots,\left(m_{2 k_{\mathrm{p}}}, y_{2 k_{\mathrm{p}}}\right)\right)$ except for the first of the two Pfaffians, which is antisymmetric

\footnotetext{
${ }^{1}$ There is an apparent singularity in this formula, because $\mathfrak{u}_{\mathrm{p}}\left(y_{a}, n_{a}\right)$ has simple poles at $y_{a}= \pm 1$ and $y_{a}$ lives in a circle of radius 1 . This is resolved by noting that, in view of (2.32), the first Pfaffian on the third line has a factor $\left(\tau^{m_{2 a} / 2} y_{2 a}-\tau^{m_{2 a-1} / 2} y_{2 a-1}\right) /\left(\tau^{\left(m_{2 a-1}+m_{2 a}\right) / 2} y_{2 a-1} y_{2 a}-1\right)$, which has zeros at these points when $m_{2 a-1}=m_{2 a}$ and $y_{2 a}=1 / y_{2 a-1}$. In later formulas it will be less apparent how these singularities are canceled (see e.g. Proposition 4.4), but we know that this will be the case by virtue of their equivalence to (4.10).
} 
under this action, we get

$$
\begin{aligned}
& \mathfrak{Z}_{m}^{\text {flat }}\left(k_{\mathrm{u}}, k_{\mathrm{p}}\right)=\frac{1}{2^{k_{\mathrm{u}}}} \sum_{\substack{m_{1}, \ldots, m_{2 k_{\mathrm{p}}+k_{\mathrm{u}}=1} \\
m_{1}+\cdots+m_{2 k_{\mathrm{p}}+k_{\mathrm{u}}}=m}}^{\infty} \frac{1}{(2 \pi \mathrm{i})^{2 k_{\mathrm{p}}+k_{\mathrm{u}}}} \int_{C_{0,1}^{2 k_{\mathrm{p}}+k_{\mathrm{u}}}} d \vec{y} \prod_{1 \leq a<b \leq 2 k_{\mathrm{p}}+k_{\mathrm{u}}} \tau^{-\frac{1}{2} m_{a} m_{b}} \\
& \times \prod_{a=1}^{2 k_{\mathrm{p}}+k_{\mathrm{u}}} \mathfrak{u}\left(y_{a}, m_{a}\right) \prod_{a=2 k_{\mathrm{p}}+1}^{2 k_{\mathrm{p}}+k_{\mathrm{u}}} \mathfrak{u}_{\mathrm{u}}\left(y_{a}, m_{a}\right)\left(\delta_{y_{a}-1}+\delta_{y_{a}+1}\right) \\
& \times \operatorname{Pf}\left[\left(-y_{a} y_{b}\right)^{m_{a} \wedge m_{b}} \operatorname{sgn}\left(y_{b} m_{b}-y_{a} m_{a}\right)\right]_{a, b=2 k_{\mathrm{p}}+1}^{2 k_{\mathrm{p}}+k_{\mathrm{u}}} \operatorname{Pf}\left[\frac{\tau^{\frac{1}{2} m_{b}} y_{b}-\tau^{\frac{1}{2} m_{a}} y_{a}}{\tau^{\frac{1}{2}\left(m_{a}+m_{b}\right)} y_{a} y_{b}-1}\right]_{a, b=1}^{2 k_{\mathrm{p}}+k_{\mathrm{u}}} \\
& \times \frac{1}{2^{k_{\mathrm{p}}}} \sum_{\pi \in T_{2 k_{\mathrm{p}}}} \operatorname{sgn}(\pi) \prod_{a=1}^{k_{\mathrm{p}}} \mathfrak{u}_{\mathrm{p}}\left(y_{\pi(2 a-1)}, m_{\pi(2 a-1)}\right) \delta_{y_{\pi(2 a-1)}-\frac{1}{y_{\pi(2 a)}}} \mathbf{1}_{m_{\pi(2 a-1)}=m_{\pi(2 a)}} .
\end{aligned}
$$

Observe that the product of the delta and indicator functions in the last line is invariant under exchanging any pair of indices of the form $(2 a-1,2 a), a=1, \ldots, k_{\mathrm{p}}$. On the other hand, we have

$$
\mathfrak{u}_{\mathrm{p}}(y, m)-\mathfrak{u}_{\mathrm{p}}(1 / y, m)=(-1)^{m} \tau^{-\frac{1}{2} m} \frac{1+y^{2}}{1-y^{2}}=\mathfrak{u}_{\mathrm{ap}}(y, m),
$$

where $\mathfrak{u}_{\text {ap }}$ was defined in (4.7), and thus it is not hard to see that

$$
\text { (last line }(4.11))=\frac{1}{2^{k_{\mathrm{p}}}} \prod_{a=1}^{k_{\mathrm{p}}} \mathfrak{u}_{\mathrm{ap}}\left(y_{2 a-1}, m_{2 a-1}\right) \delta_{y_{2 a-1}-1 / y_{2 a}} \mathbf{1}_{m_{2 a-1}=m_{2 a}} .
$$

Using this and repeating the procedure we just used of using the symmetry of the integration measure to sum over permutations, the last formula for $\mathfrak{Z}_{m}^{\text {flat }}\left(k_{\mathrm{u}}, k_{\mathrm{p}}\right)$ can be rewritten using the replacement

(last line (4.11)) $\sum_{\sigma \in S_{2 k_{\mathrm{p}}}} \frac{\operatorname{sgn}(\sigma)}{2^{k_{\mathrm{p}}\left(2 k_{\mathrm{p}}\right) !}} \prod_{a=1}^{k_{\mathrm{p}}} \mathfrak{u}_{\mathrm{ap}}\left(y_{\sigma(2 a-1)}, m_{\sigma(2 a-1)}\right) \delta_{y_{\sigma(2 a-1)}-\frac{1}{y_{\sigma(2 a)}}} \mathbf{1}_{m_{\sigma(2 a-1)}=m_{\sigma(2 a)}}$. Since, by (4.12), the matrix $\left[\mathfrak{u}_{\mathrm{ap}}\left(y_{a}, y_{b} ; m_{a}, m_{b}\right) \mathbf{1}_{m_{a}=m_{b}} \delta_{y_{a}-1 / y_{b}} \mathbf{1}_{a \neq b}\right]_{a, b=1}^{2 k_{\mathrm{p}}}$ is skew-symmetric, this sum equals $\frac{k_{\mathrm{p}} !}{\left(2 k_{\mathrm{p}}\right) !}$ times the Pfaffian of this matrix. Thus, using in addition the identity $\prod_{a=1}^{2 n} \lambda_{a} \operatorname{Pf}[A(a, b)]_{a, b=1}^{2 n}=\operatorname{Pf}\left[\lambda_{a} \lambda_{b} A(a, b)\right]_{a, b=1}^{2 n}$ (see (B.4)), the last sum can be written as

$$
\frac{1}{2^{2 k_{\mathrm{p}}}} \frac{k_{\mathrm{p}} !}{\left(2 k_{\mathrm{p}}\right) !} \operatorname{Pf}\left[4 \mathfrak{u}_{\mathrm{ap}}\left(y_{a}, m_{a}\right) \mathbf{1}_{m_{a}=m_{b}} \delta_{y_{a}-\frac{1}{y_{b}}} \mathbf{1}_{a \neq b}\right]_{a, b=1}^{2 k_{\mathrm{p}}}
$$

(the introduction of the factor $2^{-2 k_{\mathrm{p}}}$ in front of the Pfaffian is for later convenience), and now using the same Pfaffian identity again we deduce the following

Proposition 4.3. Assume that $k_{\mathrm{u}}$ is even. Then

$$
\begin{gathered}
\mathfrak{Z}_{m}^{\text {flat }}\left(k_{\mathrm{u}}, k_{\mathrm{p}}\right)=\frac{k_{\mathrm{p}} !}{2^{k_{\mathrm{u}}+2 k_{\mathrm{p}}\left(2 k_{\mathrm{p}}\right) !}} \sum_{m_{1}, \ldots, m_{2 k_{\mathrm{p}}+k_{\mathrm{u}}}=1,}^{\infty} \frac{1}{(2 \pi \mathrm{i})^{2 k_{\mathrm{p}}+k_{\mathrm{u}}}} \int_{C_{0,1}^{2 k_{\mathrm{p}}+k_{\mathrm{u}}}} d \vec{y} \prod_{1 \leq a<b \leq 2 k_{\mathrm{p}}+k_{\mathrm{u}}} \tau^{-\frac{1}{2} m_{a} m_{b}} \\
\times \prod_{a=1}^{2 k_{\mathrm{p}}+k_{\mathrm{u}}} \mathfrak{u}\left(y_{a}, m_{a}\right) \operatorname{Pf}\left[\frac{\tau^{\frac{1}{2} m_{b}} y_{b}-\tau^{\frac{1}{2} m_{a}} y_{a}}{\tau^{\frac{1}{2}\left(m_{a}+m_{b}\right)} y_{a} y_{b}-1}\right]_{a, b=1}^{2 k_{\mathrm{p}}+k_{\mathrm{u}}} \operatorname{Pf}\left[4 \mathfrak{u}_{\mathrm{ap}}\left(y_{a}, m_{a}\right) \mathbf{1}_{m_{a}=m_{b}} \delta_{y_{a}-\frac{1}{y_{b}}} \mathbf{1}_{a \neq b}\right]_{a, b=1}^{2 k_{\mathrm{p}}} \\
\times \operatorname{Pf}\left[\left(-y_{a} y_{b}\right)^{m_{a} \wedge m_{b}} \operatorname{sgn}\left(y_{b} m_{b}-y_{a} m_{a}\right) \mathfrak{u}_{\mathrm{u}}\left(y_{a}, m_{a}\right) \mathfrak{u}_{\mathrm{u}}\left(y_{b}, m_{b}\right)\right. \\
\left.\times\left(\delta_{y_{a}-1}+\delta_{y_{a}+1}\right)\left(\delta_{y_{b}-1}+\delta_{y_{b}+1}\right)\right]_{a, b=2 k_{\mathrm{p}}+1}^{2 k_{\mathrm{p}}+k_{\mathrm{u}}} .
\end{gathered}
$$


Note that the product of delta functions in the last line of (4.13) poses no difficulty when $a \neq b$ (since they involve different variables), while for $a=b$ the prefactor $\operatorname{sgn}\left(y_{b} m_{b}-y_{a} m_{a}\right)$ vanishes and thus the whole factor is interpreted as 0 .

Recalling now the definition of $\nu_{k, m}^{\text {flat }}(t)$ in Theorem 3.6 we have

$$
\nu_{k, m}^{\mathrm{flat}}(t)=\sum_{k_{\mathrm{u}}, k_{\mathrm{p}} \geq 0, k_{\mathrm{u}}+2 k_{\mathrm{p}}=k} \frac{1}{2^{k}\left(2 k_{\mathrm{p}}\right) ! k_{\mathrm{u}} !}(\star),
$$

where $(\star)$ stands for the expression starting from the first sum in (4.13). Note that in this expression the only factor which depends directly on $k_{\mathrm{u}}$ or $k_{\mathrm{p}}$ (as opposed to $k_{\mathrm{u}}+2 k_{\mathrm{p}}=k$ ) is the second Pfaffian in (4.13). Remarkably, this Pfaffian can be combined with the third Pfaffian in (4.13) and with the sum over $k_{\mathrm{u}}$ and $k_{\mathrm{p}}$ in (4.14), thus yielding an expression which only depends on $k$ (since, furthermore, the combinatorial prefactors also combine into something which only depends on $k$ ). This is done using Lemma B.2, and the result is the following: for $m \geq k \geq 0, k$ even,

$$
\begin{gathered}
\nu_{k, m}^{\text {flat }}(t)=\frac{(-1)^{\frac{1}{2} k}}{2^{k} k !} \sum_{\substack{m_{1}, \ldots, m_{k}=1, m_{1}+\cdots+m_{k}=m}}^{\infty} \sum_{y_{1}, \ldots, y_{k} \in\{-1,1\}} \frac{1}{(2 \pi \mathrm{i})^{k}} \int_{C_{0,1}^{k}} d \vec{y} \prod_{1 \leq a<b \leq 2 k_{\mathrm{p}}+k_{\mathrm{u}}} \tau^{-\frac{1}{2} m_{a} m_{b}} \prod_{a=1}^{k} \mathfrak{u}\left(y_{a}, m_{a}\right) \\
\times \operatorname{Pf}\left[\left(-y_{a} y_{b}\right)^{m_{a} \wedge m_{b}} \operatorname{sgn}\left(y_{b} m_{b}-y_{a} m_{a}\right)\left(\delta_{y_{a}-1}+\delta_{y_{a}+1}\right)\left(\delta_{y_{b}-1}+\delta_{y_{b}+1}\right) \mathfrak{u}_{\mathrm{u}}\left(y_{a}, m_{a}\right) \mathfrak{u}_{\mathrm{u}}\left(y_{b}, m_{b}\right)\right. \\
\left.+4 \mathfrak{u}_{\mathrm{ap}}\left(y_{a}, m_{a}\right) \mathbf{1}_{m_{a}=m_{b}} \delta_{y_{a}-\frac{1}{y_{b}}} \mathbf{1}_{a \neq b}\right]_{a, b=1}^{k} \operatorname{Pf}\left[\frac{\tau^{\frac{1}{2} m_{a}} y_{a}-\tau^{\frac{1}{2} m_{b}} y_{b}}{\tau^{\frac{1}{2}\left(m_{a}+m_{b}\right)} y_{a} y_{b}-1}\right]_{a, b=1}^{k} .
\end{gathered}
$$

Notice that in the last Pfaffian we have flipped the sign of the argument, which yields the additional factor $(-1)^{k / 2}$ in front. This will be convenient later.

4.2. Extension to general $k_{\mathrm{u}}$. To handle the case when $k_{\mathrm{u}}$ is odd we use a standard trick (which goes back at least to [DeB55]) to extend Pfaffians to matrices of odd size (the same idea is used in [LDC12]). Consider the dummy variables

$$
y_{2 k_{\mathrm{p}}+k_{\mathrm{u}}+1}=1, \quad \text { and } \quad m_{2 k_{\mathrm{p}}+k_{\mathrm{u}}+1}=0 .
$$

A simple computation (together with (2.32)) shows that

$$
\prod_{1 \leq a<b \leq 2 k_{\mathrm{p}}+k_{\mathrm{u}}} \frac{y_{b}-y_{a}}{y_{a} y_{b}-1}=\prod_{1 \leq a<b \leq 2 k_{\mathrm{p}}+k_{\mathrm{u}}+1} \frac{y_{b}-y_{a}}{y_{a} y_{b}-1}=\operatorname{Pf}\left[\frac{y_{b}-y_{a}}{y_{a} y_{b}-1}\right]_{a, b=1}^{2 k_{\mathrm{p}}+k_{\mathrm{u}}+1}
$$

and, similarly (using Lemma 4.1), that

$$
\begin{aligned}
& \prod_{2 k_{\mathrm{p}}+1 \leq a<b \leq 2 k_{\mathrm{p}}+k_{\mathrm{u}}}\left(-\sigma_{a} \sigma_{b}\right)^{m_{a} \wedge m_{b}+1} \operatorname{sgn}\left(\sigma_{a} m_{a}-\sigma_{b} m_{b}\right) \\
&=\operatorname{Pf}\left[\left(-\sigma_{a} \sigma_{b}\right)^{m_{a} \wedge m_{b}} \operatorname{sgn}\left(\sigma_{b} m_{b}-\sigma_{a} m_{a}\right)\right]_{a, b=2 k_{\mathrm{p}}+1}^{2 k_{\mathrm{p}}+k_{\mathrm{u}}+1} .
\end{aligned}
$$

These formulas can be used directly as replacements for (4.5) and Lemma 4.1, and lead to a formula which reads exactly like (4.10), except that the matrices appearing inside the two Pfaffians are augmented in this fashion. This can then be extended directly to (4.13) and further to a version of (4.15). We leave the details to the reader and simply record the 
result: for odd $k_{\mathrm{u}}$ we have

$$
\begin{array}{r}
\nu_{k, m}^{\text {flat }}(t)=\frac{(-1)^{\frac{1}{2}(k+1)}}{2^{k} k !} \sum_{\substack{m_{1}, \ldots, m_{k}=1, m_{1}+\cdots+m_{k}=m}}^{\infty} \frac{1}{y_{1}, \ldots, y_{k} \in\{-1,1\}} \int_{C_{0,1}^{k}} d \vec{y} \prod_{1 \leq a<b \leq 2 k_{\mathrm{p}}+k_{\mathrm{u}}} \tau^{-\frac{1}{2} m_{a} m_{b}} \\
\times \prod_{a=1}^{k} \mathfrak{u}\left(y_{a}, m_{a}\right) \operatorname{Pf}\left[\begin{array}{cc}
A & U \\
-U^{\top} & 0
\end{array}\right] \operatorname{Pf}\left[\begin{array}{rr}
B & 1 \\
-\mathbf{1}^{\top} & 0
\end{array}\right],
\end{array}
$$

where $A$ and $B$ are, respectively, the matrices appearing inside the first and second Pfaffians in (4.15), $U$ is the vector with entries $U_{a}=\mathfrak{u}_{\mathrm{u}}\left(-1, m_{a}\right) \delta_{y_{a}+1}-\mathfrak{u}_{\mathrm{u}}\left(1, m_{a}\right) \delta_{y_{a}-1}$ and 1 represents a vector of ones of size $k_{\mathrm{u}}$ (which is formally obtained from (4.15) by augmenting the matrices in the Pfaffians using the dummy variables and computing their last row and column).

Now Lemma B.1 with $V=\mathbf{1}$ together with the identity $(-1)^{\frac{1}{2} k(k+1)}=(-1)^{\frac{1}{2} k} \mathbf{1}_{k \in 2 \mathbb{Z}}+$ $(-1)^{\frac{1}{2}(k+1)} \mathbf{1}_{k \in 2 \mathbb{Z}+1}$ allow us to write a version of (4.15) and (4.16) which is valid for $k$ even and odd:

Proposition 4.4. For any $0 \leq k \leq m$ we have

$\nu_{k, m}^{\text {flat }}(t)=\frac{(-1)^{\frac{1}{2} k(k+1)}}{2^{k} k !} \sum_{\substack{m_{1}, \ldots, m_{k}=1, m_{1}+\cdots+m_{k}=m}}^{\infty} \int_{C_{0,1}^{k}} d \vec{y} \prod_{1 \leq a<b \leq 2 k_{\mathrm{p}}+k_{\mathrm{u}}} \tau^{-\frac{1}{2} m_{a} m_{b}} \prod_{a=1}^{k} \mathfrak{u}\left(y_{a}, m_{a}\right) \operatorname{Pf}[K(\vec{y} ; \vec{m})]$ where $\nu_{k, m}^{\text {flat }}$ was defined in Proposition 3.4 and

$$
K(\vec{y} ; \vec{m})=\left[\begin{array}{cc}
{\left[K_{1,1}\left(y_{a}, y_{b} ; m_{a}, m_{b}\right)\right]_{a, b=1}^{k}} & {\left[K_{1,2}\left(y_{a}, y_{b} ; m_{a}, m_{b}\right)\right]_{a, b=1}^{k}} \\
-\left[K_{1,2}\left(y_{b}, y_{a} ; m_{b}, m_{a}\right)\right]_{a, b=1}^{k} & {\left[K_{2,2}\left(y_{a}, y_{b} ; m_{a}, m_{b}\right)\right]_{a, b=1}^{k}}
\end{array}\right]
$$

with $^{2}$

$$
\begin{aligned}
K_{1,1}\left(y_{a}, y_{b} ; m_{a}, m_{b}\right) & =4 \mathfrak{u}_{\mathrm{ap}}\left(y_{a}, m_{a}\right) \mathbf{1}_{m_{a}=m_{b}} \delta_{y_{a}-\frac{1}{y_{b}}} \mathbf{1}_{a \neq b} \\
+\left(-y_{a} y_{b}\right)^{m_{a} \wedge m_{b}} \operatorname{sgn}\left(y_{b} m_{b}-y_{a} m_{a}\right) \mathfrak{u}_{\mathrm{u}}\left(y_{a}, m_{a}\right) \mathfrak{u}_{\mathrm{u}}\left(y_{b}, m_{b}\right)\left(\delta_{y_{a}-1}+\delta_{y_{a}+1}\right)\left(\delta_{y_{b}-1}+\delta_{y_{b}+1}\right) & \\
K_{1,2}\left(y_{a}, y_{b} ; m_{a}, m_{b}\right) & =\mathfrak{u}_{\mathrm{u}}\left(-1, m_{a}\right) \delta_{y_{a}+1}-\mathfrak{u}_{\mathrm{u}}\left(1, m_{a}\right) \delta_{y_{a}-1}, \\
K_{2,2}\left(y_{a}, y_{b} ; m_{a}, m_{b}\right) & =\frac{\tau^{\frac{1}{2} m_{a}} y_{a}-\tau^{\frac{1}{2} m_{b}} y_{b}}{\tau^{\frac{1}{2}\left(m_{a}+m_{b}\right)} y_{a} y_{b}-1}
\end{aligned}
$$

where $\mathfrak{u}, \mathfrak{u}_{\mathrm{ap}}$, and $\mathfrak{u}_{\mathrm{u}}$ are given in (4.7).

This result together with (2.20), (2.25) and Proposition 3.4 yield the following explicit formula for the exponential moments of flat ASEP: for any $m \in \mathbb{Z}_{\geq 0}$ we have, with $K$ as in Proposition 4.4,

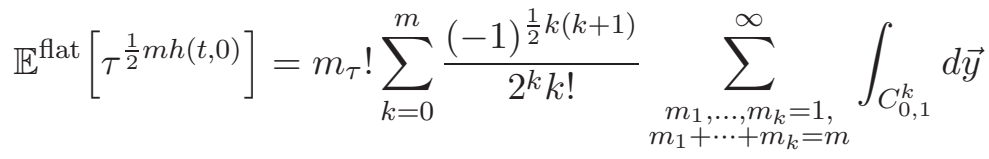

$$
\begin{aligned}
& \times \prod_{1 \leq a<b \leq k} \tau^{-\frac{1}{2} m_{a} m_{b}} \prod_{a=1}^{k} \mathfrak{u}\left(y_{a}, m_{a}\right) \operatorname{Pf}[K(\vec{y} ; \vec{m})] .
\end{aligned}
$$

\footnotetext{
${ }^{2}$ In order to avoid an even heavier notation, we are slightly abusing it in the definition of $K_{1,1}\left(y_{a}, y_{b} ; m_{a}, m_{b}\right)$, which depends on $a$ and $b$ explicitly in addition to $y_{a}, y_{b}, m_{a}$ and $m_{b}$. This will become irrelevant in later version of our formulas, see footnote 4 in page 29.
} 
At this point we can get rid of the delta functions inside $K$, by rewriting (4.18) in such a way that we can take the $y_{a}$ integrations inside the Pfaffian. To this end, let $p_{a}=\frac{1-\tau^{m_{a} / 2} y_{a}}{1+\tau^{m_{a} / 2} y_{a}}$, so that $\frac{\tau^{m_{a} / 2} y_{a}-\tau^{m_{b} / 2} y_{b}}{\tau^{\left(m_{a}+m_{b}\right) / 2} y_{a} y_{b}-1}=\frac{p_{a}-p_{b}}{p_{a}+p_{b}}$. Note that $\Re\left(p_{a}\right)>0$. The key will be to use the identity

$$
\frac{p_{a}-p_{b}}{p_{a} p_{b}\left(p_{a}+p_{b}\right)}=\int_{\left(\mathbb{R}_{\geq 0}\right)^{2}} d \lambda_{a} d \lambda_{b} e^{-\lambda_{a} p_{a}-\lambda_{b} p_{b}} \operatorname{sgn}\left(\lambda_{b}-\lambda_{a}\right)
$$

In order to use it, we first use the Pfaffian identity (B.4) to rewrite (4.18) as

$$
\begin{aligned}
\mathbb{E}^{\mathrm{fl}}\left[\tau^{\frac{1}{2} m h(t, 0)}\right]=m_{\tau} ! \sum_{k=0}^{m} \frac{1}{2^{k} k !} \sum_{\substack{m_{1}, \ldots, m_{k}=1, m_{1}+\cdots+m_{k}=m}}^{\infty} \frac{1}{(2 \pi \mathrm{i})^{k}} \int_{C_{0,1}^{k}} d \vec{y} \prod_{1 \leq a<b \leq k} \tau^{-\frac{1}{2} m_{a} m_{b}} \prod_{a} \mathfrak{u}\left(y_{a}, m_{a}\right) p_{a} \\
\times(-1)^{\frac{1}{2} k(k+1)} \operatorname{Pf}\left[\begin{array}{cc}
{\left[K_{1,1}\left(y_{a}, y_{b} ; m_{a}, m_{b}\right)\right]_{a, b=1}^{k}} & {\left[\frac{1}{p_{b}} K_{1,2}\left(y_{a}, y_{b} ; m_{a}, m_{b}\right)\right]_{a, b=1}^{k}} \\
-\left[\frac{1}{p_{a}} K_{1,2}\left(y_{b}, y_{a} ; m_{b}, m_{a}\right)\right]_{a, b=1}^{k} & {\left[\frac{p_{a}-p_{b}}{p_{a} p_{b}\left(p_{a}+p_{b}\right)}\right]_{a, b=1}^{k}}
\end{array}\right] .
\end{aligned}
$$

Now using (4.19) and Lemma B.3, which is a certain Pfaffian version of the Andréief (or generalized Cauchy-Binet) identity [And83] (more precisely, we use a version of the lemma with the matrices inside the two Pfaffians transposed), with respect to the integration in the $\lambda_{a}$ 's, with $\phi_{a}\left(\lambda_{a}\right)=e^{-\lambda_{a} p_{a}}$, we get

$$
\begin{aligned}
\mathbb{E}^{\text {flat }}\left[\tau^{\frac{1}{2} m h(t, 0)}\right]=m_{\tau} ! \sum_{k=0}^{m} \frac{1}{k !} \sum_{\substack{m_{1}, \ldots, m_{k}=1 \\
m_{1}+\cdots+m_{k}=m}}^{\infty} \frac{1}{(2 \pi \mathrm{i})^{k}} \int_{C_{0,1}^{k}} d \vec{y} \int_{\left(\mathbb{R}_{\geq 0}\right)^{k}} d \vec{\lambda} \\
\times \prod_{a=1}^{k} \mathfrak{u}\left(y_{a}, m_{a}\right) p_{a} e^{-\lambda_{a} p_{a}} \prod_{1 \leq a<b \leq k} \tau^{-\frac{1}{2} m_{a} m_{b}} \\
\times \frac{(-1)^{\frac{1}{2} k(k+1)}}{2^{k}} \operatorname{Pf}\left[\begin{array}{cc}
{\left[K_{1,1}\left(y_{a}, y_{b} ; m_{a}, m_{b}\right)\right]_{a, b=1}^{k}} & {\left[K_{1,2}\left(y_{a}, y_{b} ; m_{a}, m_{b}\right)\right]_{a, b=1}^{k}} \\
-\left[K_{1,2}\left(y_{b}, y_{a} ; m_{b}, m_{a}\right)\right]_{a, b=1}^{k} & {\left[\operatorname{sgn}\left(\lambda_{b}-\lambda_{a}\right)\right]_{a, b=1}^{k}}
\end{array}\right] .
\end{aligned}
$$

We would like next to interchange the $y_{a}$ and $\lambda_{a}$ integrals. The difficulty we face is that the $y_{a}$ contours pass through singularities of the factors $\mathfrak{u}_{\mathrm{ap}}\left(y_{a}, m_{a}\right)$ in the integrand, and after interchanging the integrals the zeros of the second Pfaffian in (4.11) (see footnote 1 in page 22) are not there any more cancel them. This means in particular that the $y_{a}$ integrals that result after the interchange will have to be regarded as principal value integrals. In order to justify the interchange of limits let us restrict ourselves to the case $k=2$. The general case can be justified similarly but the notation becomes much heavier.

When $k=2$ we may use the Pfaffian formula

$$
\operatorname{Pf}\left[\begin{array}{cccc}
0 & a & b & c \\
-a & 0 & d & e \\
-b & -d & 0 & f \\
-c & -e & -f & 0
\end{array}\right]=a f-b e+d c
$$

to write the double integral in (4.20) as

$$
\begin{aligned}
\frac{-1}{4(2 \pi \mathrm{i})^{2}} \int_{C_{0,1}^{2}} d \vec{y} \int_{\left(\mathbb{R}_{\geq 0}\right)^{2}} d \vec{\lambda} \mathfrak{u}\left(y_{1}, m_{1}\right) \mathfrak{u}\left(y_{2}, m_{2}\right) p_{1} p_{2} e^{-\lambda_{1} p_{1}-\lambda_{2} p_{2}} \tau^{-\frac{1}{2} m_{1} m_{2}} & \\
\times\left[K_{1,1}\left(y_{1}, y_{2} ; m_{1}, m_{2}\right) \operatorname{sgn}\left(\lambda_{2}-\lambda_{1}\right)-\right. & K_{1,2}\left(y_{1}, y_{1} ; m_{1}, m_{1}\right) K_{1,2}\left(y_{2}, y_{2} ; m_{2}, m_{2}\right) \\
& \left.+K_{1,2}\left(y_{1}, y_{2} ; m_{1}, m_{2}\right) K_{1,2}\left(y_{2}, y_{1} ; m_{2}, m_{1}\right)\right] .
\end{aligned}
$$

The last two terms in the bracket pose no difficulty. In fact, recalling that $K_{1,2}\left(y, y^{\prime} ; m, m^{\prime}\right)$ only depends on $y$ and $m$, each of the two terms involves products of delta functions depending on different variables, and one can check directly that the $y_{1}, y_{2}$ integration 
(which corresponds to summing the values at \pm 1 ) can be done before or after the $\lambda_{1}, \lambda_{2}$ integration.

Now consider the first term in the bracket above and call $I_{1}$ the associated integral. Integrating $\lambda_{1}, \lambda_{2}$ first and using (4.19) yields

$$
I_{1}=\frac{-1}{4(2 \pi \mathrm{i})^{2}} \int_{C_{0,1}^{k}} d \vec{y} \mathfrak{u}\left(y_{1}, m_{1}\right) \mathfrak{u}\left(y_{2}, m_{2}\right) \tau^{-\frac{1}{2} m_{1} m_{2}} \frac{p_{1}-p_{2}}{p_{1}+p_{2}} K_{1,1}\left(y_{1}, y_{2} ; m_{1}, m_{2}\right) .
$$

$K_{1,1}$ is defined in (4.17) and has two terms. Call $I_{1,1}$ and $I_{1,2}$ the integrals associated to each (so that $I_{1}=I_{1,1}+I_{1,2}$ ). As before, the second integral poses no difficulty for the interchange of $\lambda_{a}$ and $y_{a}$ integrals, so let us focus on $I_{1,1}$. Here, thanks to the prefactor $\frac{p_{1}-p_{2}}{p_{1}+p_{2}}$, the integrand has no singularities on the $y_{a}$ contours and thus the integration simply yields

$I_{1,1}=\frac{-1}{2 \pi \mathrm{i}} \int_{C_{0,1}} d y \mathfrak{u}\left(y, m_{1}\right) \mathfrak{u}\left(\frac{1}{y}, m_{1}\right) \mathfrak{u}_{\mathrm{ap}}\left(y, m_{1}\right) \mathfrak{u}_{\mathrm{ap}}\left(\frac{1}{y}, m_{1}\right) \frac{p\left(y, m_{1}\right)-p\left(\frac{1}{y}, m_{1}\right)}{p\left(y, m_{1}\right)+p\left(\frac{1}{y}, m_{1}\right)} \tau^{-\frac{1}{2} m_{1}^{2}} \mathbf{1}_{m_{1}=m_{2}}$

with $p(y, m)=\frac{1-\tau^{m / 2} y}{1+\tau^{m / 2} y}$.

Next we let let $\widetilde{I}_{1,1}$ be the analogous integral where we now integrate first in $\vec{y}$. In this case the integrand has singularities along the $\vec{y}$ contour, so we will have to regard this as a principal value integral while dealing with the delta functions at the same time. More precisely, letting $C_{0,1}^{\delta}$ be portion of the contour $C_{0,1}$ which lies outside of the circles of radius $\delta$ centred at \pm 1 , and letting $\varphi_{\varepsilon}$ be a suitable mollifier, we define $\widetilde{I}_{1,1}$ as

$$
\begin{array}{r}
\widetilde{I}_{1,1}=\lim _{\varepsilon \rightarrow 0} \lim _{\delta_{1}, \delta_{2} \rightarrow 0} \frac{-1}{(2 \pi \mathrm{i})^{2}} \int_{\left(\mathbb{R}_{\geq 0}\right)^{2}} d \vec{\lambda} \int_{C_{0,1}^{\delta_{1}} \times C_{0,1}^{\delta_{2}}} d \vec{y} \mathfrak{u}\left(y_{1}, m_{1}\right) \mathfrak{u}\left(y_{2}, m_{1}\right) \mathfrak{u}_{\mathrm{ap}}\left(y_{1}, m_{1}\right) \mathfrak{u}_{\mathrm{ap}}\left(y_{2}, m_{1}\right) \\
\times \tau^{-\frac{1}{2} m_{1}^{2}} p_{1} p_{2} e^{-\lambda_{1} p_{1}-\lambda_{2} p_{2}} \operatorname{sgn}\left(\lambda_{2}-\lambda_{1}\right) \varphi_{\varepsilon}\left(y_{1}-1 / y_{2}\right) .
\end{array}
$$

Using Cauchy's Theorem, each of the $\delta_{a} \rightarrow 0$ limits equals the integral over a circle $\widetilde{C}_{0,1}$ of radius slightly smaller than 1 plus half the residues of the integrand at $y_{a}= \pm 1$. Using this to compute the two limits (one after the other) leads, after some calculation, to

$$
\begin{aligned}
& \widetilde{I}_{1,1}=-\lim _{\varepsilon \rightarrow 0} \frac{1}{(2 \pi \mathrm{i})^{2}} \int_{\left(\mathbb{R}_{\geq 0}\right)^{2}} d \vec{\lambda} \int_{\left(\widetilde{C}_{0,1}\right)^{2}} d \vec{y} \tau^{-\frac{1}{2} m_{1}^{2}} \mathfrak{u}\left(y_{1}, m_{1}\right) \mathfrak{u}\left(y_{2}, m_{1}\right) \mathfrak{u}_{\mathrm{ap}}\left(y_{1}, m_{1}\right) \mathfrak{u}_{\mathrm{ap}}\left(y_{2}, m_{1}\right) \\
& \times p_{1} p_{2} e^{-\lambda_{1} p_{1}-\lambda_{2} p_{2}} \operatorname{sgn}\left(\lambda_{2}-\lambda_{1}\right) \varphi_{\varepsilon}\left(y_{1}-1 / y_{2}\right) \\
&- \lim _{\varepsilon \rightarrow 0} \sum_{\sigma \in\{-1,1\}} \frac{1}{2 \pi \mathrm{i}} \int_{\left(\mathbb{R}_{\geq 0}\right)^{2}} d \vec{\lambda} \int_{\widetilde{C}_{0,1}} d y \tau^{-\frac{1}{2} m_{1}^{2}} \mathfrak{u}\left(y, m_{1}\right) \mathfrak{u}\left(\sigma, m_{1}\right) \mathfrak{u}_{\mathrm{ap}}\left(y, m_{1}\right) \sigma_{2}(-1)^{m_{1}} \\
& \quad \times \tau^{-\frac{1}{2} m_{1}} p\left(y, m_{1}\right) p\left(\sigma_{2}, m_{1}\right) e^{-\lambda_{1} p\left(y, m_{1}\right)-\lambda_{2} p\left(\sigma_{2}, m_{1}\right)} \operatorname{sgn}\left(\lambda_{2}-\lambda_{1}\right) \varphi_{\varepsilon}(y-\sigma) \\
&-\lim _{\varepsilon \rightarrow 0} \frac{1}{4} \sum_{\sigma_{1}, \sigma_{2} \in\{-1,1\}} \int_{\left(\mathbb{R}_{\geq 0}\right)^{2}} d \vec{\lambda} \tau^{-\frac{1}{2} m_{1}^{2}} \mathfrak{u}\left(\sigma_{1}, m_{1}\right) \mathfrak{u}\left(\sigma_{2}, m_{1}\right) \sigma_{1} \sigma_{2} \tau^{-m_{1}} \\
& \quad \times p\left(\sigma_{1}, m_{1}\right) p\left(\sigma_{2}, m_{1}\right) e^{-\lambda_{1} p\left(\sigma_{1}, m_{1}\right)-\lambda_{2} p\left(\sigma_{2}, m_{1}\right)} \operatorname{sgn}\left(\lambda_{2}-\lambda_{1}\right) \varphi_{\varepsilon}\left(\sigma_{1}-\sigma_{2}\right) .
\end{aligned}
$$

At this point we can perform the $\lambda_{a}$ integrals using (4.19) (note that in the first two terms there is now no difficulty in interchanging the $\lambda_{a}$ and $y_{a}$ integrations). Starting with the last one, it yields a factor $\frac{p\left(\sigma_{1}, m_{1}\right)-p\left(\sigma_{2}, m_{1}\right)}{p\left(\sigma_{1}, m_{1}\right)+p\left(\sigma_{2}, m_{1}\right)}$; when $\sigma_{1}=\sigma_{2}$ this factor vanishes, while when $\sigma_{1} \neq \sigma_{2}$ the limit $\lim _{\varepsilon \rightarrow 0} \varphi_{\varepsilon}\left(\sigma_{1}-\sigma_{2}\right)$ vanishes. This means that the third limit above is zero. One can check similarly that the second limit vanishes, by performing the $\lambda_{a}$ integrations to obtain a factor $\frac{p\left(\sigma, m_{1}\right)-p\left(y, m_{1}\right)}{p\left(\sigma, m_{1}\right)+p\left(y, m_{1}\right)}$ and then computing the $\varepsilon \rightarrow 0$ limit to yield evaluation at $y=\sigma$, for which this factor vanishes. Performing the $\lambda_{a}$ integrals now in the first term and taking $\varepsilon \rightarrow 0$ shows that $\widetilde{I}_{1,1}$ equals the right hand side of (4.21) with the contour $C_{0,1}$ 
on the right hand side replaced by $\widetilde{C}_{0,1}$. But since the integrand has no singularities along $C_{0,1}$, Cauchy's Theorem implies that $I_{1,1}=\widetilde{I}_{1,1}$ as desired.

The conclusion of all this (and its extension to all $k \geq 1$ ) is that we may interchange the $\lambda_{a}$ and $y_{a}$ integrations in (4.20), provided we mollify the delta functions and we interpret the $y_{a}$ integrals as principal value integrals as done in (4.22). Now observe that in the Pfaffian appearing in $(4.20)$, the $(1,2)$ entry only depends on $y_{a}$, the $(2,1)$ entry only depends on $y_{b}$, and the $(2,2)$ entry depends on neither of the two. Hence we can use Lemma B.3 again, this time to bring the $y_{a}$ integrals inside the Pfaffian. Let us first define

$$
\begin{aligned}
& \mathfrak{v}\left(\lambda_{a}, y_{a}, m_{a}\right)= p_{a} e^{-\lambda_{a} p_{a}} \mathfrak{u}\left(y_{a}, m_{a}\right) \\
&=\frac{1}{y_{a}}(1-\tau)^{m_{a}} \tau^{m_{a} / 2} \frac{1-\tau^{m_{a} / 2} y_{a}}{1+\tau^{m_{a} / 2} y_{a}} e^{-\lambda_{a} \frac{1-\tau^{m_{a} / 2} y_{a}}{1+\tau_{a} / 2} y_{a}}+t\left[\frac{1}{1+\tau^{-m_{a} / 2} y_{a}}-\frac{1}{1+\tau^{m_{a} / 2} y_{a}}\right] \\
& \quad \times \frac{\left(-\tau^{-m_{a} / 2} y ; \tau\right)_{\infty}}{\left(-\tau^{m_{a} / 2} y_{a} ; \tau\right)_{\infty}} \frac{\left(\tau^{1+m_{a}} y^{2} ; \tau\right)_{\infty}}{\left(\tau y_{a}^{2} ; \tau\right)_{\infty}}
\end{aligned}
$$

where we have used the definition of $\mathfrak{u}$ in (4.7) (and of $\mathfrak{f}_{1}$ in (2.23)), and observe that the middle line of (4.20) can be written as $\tau^{-m^{2} / 4} \prod_{a} \tau^{\frac{1}{4} m_{a}^{2}} \mathfrak{v}\left(\lambda_{a}, y_{a}, m_{a}\right)$ (recall that $\sum_{a} m_{a}=$ $m)$. Define also ${ }^{3}$

$$
\begin{aligned}
& K_{1,1}^{\mathrm{flat}}\left(\lambda_{a}, \lambda_{b} ; m_{a}, m_{b}\right)=\frac{1}{2(2 \pi \mathrm{i})^{2}} f_{C_{0,1}^{2}} d y d y^{\prime} \tau^{\frac{1}{4}\left(m_{a}^{2}+m_{b}^{2}\right)} \mathfrak{v}\left(\lambda_{a}, y, m_{a}\right) \mathfrak{v}\left(\lambda_{b}, y^{\prime}, m_{b}\right) \\
& \times K_{1,1}\left(y, y^{\prime} ; m_{a}, m_{b}\right) \\
& =\mathbf{1}_{m_{a}=m_{b}} \frac{1}{\pi \mathrm{i}} \int_{C_{0,1}} d y \tau^{\frac{1}{2} m_{a}^{2}} \mathfrak{v}\left(\lambda_{a}, y, m_{a}\right) \mathfrak{v}\left(\lambda_{b}, 1 / y, m_{b}\right) \mathfrak{u}_{\mathrm{ap}}\left(y, m_{a}\right) \\
& +\frac{1}{2} \sum_{\sigma, \sigma^{\prime} \in\{-1,1\}}\left(-\sigma \sigma^{\prime}\right)^{m_{a} \wedge m_{b}+1} \operatorname{sgn}\left(\sigma^{\prime} m_{b}-\sigma m_{a}\right) \\
& \times \tau^{\frac{1}{4}\left(m_{a}^{2}+m_{b}^{2}\right)-\frac{1}{2}\left(m_{a}+m_{b}\right)} \mathfrak{v}\left(\lambda_{a}, \sigma, m_{a}\right) \mathfrak{v}\left(\lambda_{b}, \sigma^{\prime}, m_{b}\right), \\
& K_{1,2}^{\mathrm{flat}}\left(\lambda_{a}, \lambda_{b} ; m_{a}, m_{b}\right)=\frac{1}{4 \pi \mathrm{i}} \int_{C_{0,1}} d y \tau^{\frac{1}{4} m_{a}^{2}} \mathfrak{v}\left(\lambda_{a}, y, m_{a}\right) K_{1,2}\left(y,-; m_{a},-\right) \\
& =-\frac{1}{2} \sum_{\sigma \in\{-1,1\}} \tau^{\frac{1}{4} m_{a}^{2}-\frac{1}{2} m_{a}} \mathfrak{v}\left(\lambda_{a}, \sigma, m_{a}\right), \\
& K_{2,2}^{\mathrm{flat}}\left(\lambda_{a}, \lambda_{b} ; m_{a}, m_{b}\right)=\frac{1}{2} \operatorname{sgn}\left(\lambda_{b}-\lambda_{a}\right)
\end{aligned}
$$

where $\mathfrak{u}_{\mathrm{ap}}$ is given in (4.7), $\mathfrak{v}$ is given in (4.23) (the -'s in $K_{1,2}$ denote the fact that this kernel does not depend on those arguments), and we have used the fact that for $\sigma \in\{-1,1\}$ the function $\mathfrak{u}_{\mathfrak{u}}(\sigma, m)$ (defined in (4.7)) satisfies $\mathfrak{u}_{\mathfrak{u}}(\sigma, m)=\tau^{-\frac{m}{2}} \sigma \frac{1-\tau^{n} \sigma^{2}}{1-\tau^{n}}=\sigma \tau^{-\frac{m}{2}}$. Then, by virtue of Lemma B.3 again, we get

$$
\begin{aligned}
&\left.\mathbb{E}^{\mathrm{flat}}\left[\tau^{\frac{1}{2} m h(t, 0)}\right)\right]=m_{\tau} ! \tau^{-\frac{1}{4} m^{2}} \sum_{k=0}^{m} \frac{1}{k !} \sum_{\substack{m_{1}, \ldots, m_{k}=1, m_{1}+\cdots+m_{k}=m}}^{\infty} \int_{\left(\mathbb{R}_{\geq 0}\right)^{k}} d \vec{\lambda} \\
& \times(-1)^{\frac{1}{2} k(k+1)} \operatorname{Pf}\left[\begin{array}{l}
{\left[K_{1,1}^{\mathrm{flat}}\left(\lambda_{a}, \lambda_{b} ; m_{a}, m_{b}\right)\right]_{a, b=1}^{k}} \\
\left.-\left[K_{1,2}^{\mathrm{flat}}\left(\lambda_{b}, \lambda_{a} ; m_{b}, m_{a}\right)\right]_{a, b=1}^{k}\left[K_{1,2}^{\mathrm{fat}}\left(\lambda_{a}, \lambda_{b} ; m_{a}, m_{b}\right)\right]_{a, b=1}^{k}\left(\lambda_{a}, \lambda_{b} ; m_{a}, m_{b}\right)\right]_{a, b=1}^{k}
\end{array}\right] .
\end{aligned}
$$

(Note that we have used the fact that $\operatorname{Pf}[a A]=a^{n} \operatorname{Pf}[A]$ for a skew-symmetric $2 n \times 2 n$ matrix $A$ and a scalar $a$. See (B.3).)

\footnotetext{
${ }^{3}$ The notation $f$ in the second line of this formula indicates that this is a Cauchy principal value integral.
} 


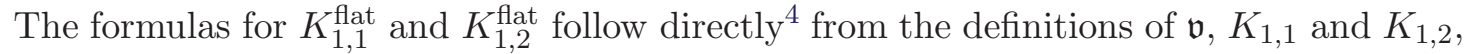
with the nuisance that we have to justify writing a principal value integral in $K_{1,1}^{\text {flat }}$. This involves writing out explicitly the result of the interchange of the $\lambda_{a}$ and $y_{a}$ integrations in (4.20) using mollifiers and principal value integrals in a similar way as (4.22), then moving the $y_{a}$ integrals inside the Pfaffian, and finally showing that the limits involving the mollification and the principal value integrals can be taken back inside the Pfaffian to yield the kernel $K^{\text {flat }}$. The argument is very similar to the one we used to justify the interchange of limits itself, so we omit it.

The factor $(-1)^{\frac{1}{2} k(k+1)}$ in front of the Pfaffian in (4.25) now allows us to write the formula in the form of a Fredholm Pfaffian. In fact, let $K^{\text {flat }}$ be the $2 \times 2$ matrix kernel

$$
K^{\mathrm{flat}}\left(\lambda_{a}, \lambda_{b} ; m_{a}, m_{b}\right)=\left[\begin{array}{cc}
K_{1,1}^{\mathrm{flat}}\left(\lambda_{a}, \lambda_{b} ; m_{a}, m_{b}\right) & K_{1,2}^{\mathrm{flat}}\left(\lambda_{a}, \lambda_{b} ; m_{a}, m_{b}\right) \\
-K_{1,2}^{\mathrm{flat}}\left(\lambda_{b}, \lambda_{a} ; m_{b}, m_{a}\right) & K_{2,2}^{\mathrm{flat}}\left(\lambda_{a}, \lambda_{b} ; m_{a}, m_{b}\right)
\end{array}\right] .
$$

The matrix $\left[K^{\text {flat }}\left(\lambda_{a}, \lambda_{b} ; m_{a}, m_{b}\right)\right]_{a, b=1}^{k}$ differs from the one inside the above Pfaffian by a permutation of the rows and columns which has sign $(-1)^{k(k-1) / 2}$, and thus we get

$$
\mathbb{E}^{\mathrm{flat}}\left[\tau^{\frac{1}{2} m h(t, 0)}\right]=m_{\tau} ! \tau^{-\frac{1}{4} m^{2}} \sum_{k=0}^{m} \frac{(-1)^{k}}{k !} \sum_{\substack{m_{1}, \ldots, m_{k}=1 \\ m_{1}+\cdots+m_{k}=m}}^{\infty} \int_{\left(\mathbb{R}_{\geq 0}\right)^{k}} d \vec{\lambda} \operatorname{Pf}\left[K^{\mathrm{flat}}\left(\lambda_{a}, \lambda_{b} ; m_{a}, m_{b}\right)\right]_{a, b=1}^{k} .
$$

This gives Theorem 2.3.

As a corollary we get a bound on the moments of $\tau^{\frac{1}{2} h(t, 0)}$, which will be useful later on when we form a generating function for $\tau^{\frac{1}{2} h(t, 0)}$ :

Corollary 4.5. There is a $c>0$ such that for all $m \in \mathbb{Z}_{\geq 1}$,

$$
\mathbb{E}^{\text {flat }}\left[\tau^{\frac{1}{2} m h(t, 0)}\right] \leq c m_{\tau} ! \tau^{-\frac{1}{4} m^{2}}
$$

We note that for fixed $\tau$ we have $m_{\tau} ! \leq c(1-\tau)^{-m}$ (this follows from the $q$-analogue of Stirling's formula for the $q$-Gamma function), and hence the above moment bound is actually of the form $c \tau^{-\frac{1}{4} m^{2}-O(m)}$. Before getting started with the proof we need to establish an estimate on certain ratios of $q$-Pochhammer symbols.

Lemma 4.6. For any $\eta>\frac{1}{8}$ and $\tau \in(0,1)$ we have

$$
\sup _{n \in \mathbb{Z}_{\geq 1},|z|=1} \tau^{\eta n^{2}} \frac{\left(-\tau^{-n / 2} z ; \tau\right)_{\infty}}{\left(-\tau^{n / 2} z ; \tau\right)_{\infty}} \frac{\left(\tau^{1+n} z^{2} ; \tau\right)_{\infty}}{\left(\tau z^{2} ; \tau\right)_{\infty}}<\infty .
$$

Proof. By definition of the $q$-Pochhamer symbol,

$$
\frac{\left(-\tau^{-n / 2} z ; \tau\right)_{\infty}}{\left(-\tau^{n / 2} z^{2} ; \tau\right)_{\infty}} \frac{\left(\tau^{1+n} z^{2} ; \tau\right)_{\infty}}{\left(\tau z^{2} ; \tau\right)_{\infty}}=\prod_{\ell=0}^{n-1} \frac{1+\tau^{\ell-n / 2} z}{1-\tau^{\ell+1} z^{2}} .
$$

It is not hard to see that the absolute value of each factor is maximized for $z$ in the unit circle at $z=1$, whence

$$
\left|\frac{\left(-\tau^{-n / 2} z ; \tau\right)_{\infty}}{\left(-\tau^{n / 2} z^{2} ; \tau\right)_{\infty}} \frac{\left(\tau^{1+n} z^{2} ; \tau\right)_{\infty}}{\left(\tau z^{2} ; \tau\right)_{\infty}}\right| \leq \frac{\prod_{\ell=0}^{n-1}\left(1+\tau^{\ell-n / 2}\right)}{\prod_{\ell=1}^{n}\left(1-\tau^{\ell}\right)} \leq \frac{1}{(\tau ; \tau)_{\infty}} \prod_{\ell=0}^{n-1}\left(1+\tau^{\ell-n / 2}\right) .
$$

Expanding the last product and bounding each term by the biggest one, which is given by $\prod_{\ell=0}^{\lfloor n / 2\rfloor} \tau^{\ell-n / 2} \approx \tau^{-n^{2} / 8}$ results in the bound

$$
\left|\frac{\left(-\tau^{-n / 2} z ; \tau\right)_{\infty}}{\left(-\tau^{n / 2} z^{2} ; \tau\right)_{\infty}} \frac{\left(\tau^{1+n} z^{2} ; \tau\right)_{\infty}}{\left(\tau z^{2} ; \tau\right)_{\infty}}\right| \leq \frac{C}{(\tau ; \tau)_{\infty}} 2^{n} \tau^{-n^{2} / 8}
$$

\footnotetext{
${ }^{4}$ Observe that we have dropped the factor $\mathbf{1}_{a \neq b}$ in the second term of the second equality in the definition of $K_{1,1}^{\text {flat }}$. We may do this because this term vanishes when $\lambda_{a}=\lambda_{b}$ and $m_{a}=m_{b}$, as can be checked using the antisymmetry of $\left(m, m^{\prime}\right) \longmapsto \operatorname{sgn}\left(m-m^{\prime}\right)$.
} 
for some $C>0$, which implies the result.

Proof of Corollary 4.5. Recall that $\mathfrak{v}\left(\lambda_{a}, y, m_{a}\right)$ contains a factor $e^{-\lambda_{a} p_{a}}$ with $p_{a}=\frac{1-\tau^{m_{a} / 2} y}{1+\tau^{m_{a} / 2} y}$ and note that $\Re\left(p_{a}\right) \geq c_{1}$ for some $c_{1}>0$, uniformly in $y \in C_{0,1}$ and $m_{a} \in \mathbb{Z}_{\geq 1}$. Having chosen $c_{1}$ in this way, we use (B.4) to factor out $\prod_{a} e^{-\lambda_{a} p_{a}} \tau^{m_{a}^{2} / 16}$ from the Pfaffian:

$$
\operatorname{Pf}\left[K^{\text {flat }}\left(\lambda_{a}, \lambda_{b} ; m_{a}, m_{b}\right)\right]_{a, b=1}^{\ell}=\prod_{a=1}^{\ell} e^{-c_{1} \lambda_{a}} \tau^{\frac{1}{16} m_{a}^{2}} \operatorname{Pf}\left[K^{\text {flat }^{\prime}}\left(\lambda_{a}, \lambda_{b} ; m_{a}, m_{b}\right)\right]_{a, b=1}^{\ell}
$$

where

$$
K^{\text {flat }^{\prime}}\left(\lambda_{a}, \lambda_{b} ; m_{a}, m_{b}\right)=\left[\begin{array}{cc}
e^{c_{1}\left(\lambda_{a}+\lambda_{b}\right)} \tau^{-\left(m_{a}^{2}+m_{b}^{2}\right) / 16} K_{1,1}\left(\lambda_{a}, \lambda_{b} ; m_{a}, m_{b}\right) & e^{c_{1} \lambda_{a}} \tau^{-m_{a}^{2} / 16} K_{1,2}\left(\lambda_{a}, \lambda_{b} ; m_{a}, m_{b}\right) \\
-e^{c_{1} \lambda_{b}} \tau^{-m_{b}^{2} / 16} K_{1,2}\left(\lambda_{b}, \lambda_{a} ; m_{b}, m_{a}\right) & K_{2,2}\left(\lambda_{a}, \lambda_{b} ; m_{a}, m_{b}\right)
\end{array}\right] .
$$

The point of rewriting $K^{\text {flat }}$ in this manner is that the entries of $K^{\text {flat }}\left(\lambda_{1}, \lambda_{2} ; m_{1}, m_{2}\right)$ are uniformly bounded for $\lambda_{1}, \lambda_{2} \geq 0$ and $m_{1}, m_{2} \in \mathbb{Z}_{\geq 1}$, say by a constant $c_{2}>0$. This can be checked straightforwardly for most of the factors involved, except for two minor issues to address. First, $\mathfrak{v}\left(\lambda_{a}, y, m_{a}\right)$ contains a factor of the form $g\left(y, m_{a}\right):=$ $\frac{\left(-\tau^{-m_{a} / 2} y ; \tau\right)_{\infty}}{\left(-\tau^{m_{a} / 2} y ; \tau\right)_{\infty}} \frac{\left(\tau^{1+m_{a}} y^{2} ; \tau\right)_{\infty}}{\left(\tau y^{2} ; \tau\right)_{\infty}}$, which is not bounded in $m_{a}$. Nevertheless, $\mathfrak{v}\left(\lambda_{a}, y, m_{a}\right)$ appears next to $\tau^{m_{a}^{2} / 4}$, which together with the factor $\tau^{-m_{a}^{2} / 16}$ introduced above gives a factor $\tau^{3 m_{a}^{2} / 16}$. Thanks to Lemma 4.6 this factor is enough to balance the growth of $g\left(y, m_{a}\right)$. The other term which is not seen directly to be bounded is the one coming from $K_{1,1}$ involving the principal value integral $\mathbf{1}_{m_{a}=m_{b}} \frac{1}{2 \pi \mathrm{i}} f_{C_{0,1}} d y \mathfrak{v}\left(\lambda_{a}, y, m_{a}\right) \mathfrak{v}\left(\lambda_{b}, 1 / y, m_{a}\right) \mathfrak{u}_{\mathrm{ap}}\left(y, m_{a}\right)$. But in this case the uniform bound follows from the fact that the integrand can be expanded around \pm 1 as $\frac{1}{1 \mp y}+\mathcal{O}(1)$.

Using the fact that $K^{\text {flat }}$ is uniformly bounded, the identity $\operatorname{Pf}[A]^{2}=\operatorname{det}[A]$ and Hadamard's bound we deduce that $\left|\operatorname{Pf}\left[K^{\text {flat }}\left(\lambda_{a}, \lambda_{b} ; m_{a}, m_{b}\right)\right]_{a, b=1}^{k}\right| \leq k^{k / 2} c_{2}^{k} \prod_{a} e^{-c_{1} \lambda_{a}} \tau^{m_{a}^{2} / 16}$, and thus

$$
\left|\int_{\left(\mathbb{R}_{\geq 0}\right)^{k}} d \vec{\lambda} \operatorname{Pf}\left[K^{\text {flat }}\left(\lambda_{a}, \lambda_{b} ; m_{a}, m_{b}\right)\right]_{a, b=1}^{k}\right| \leq\left(c_{2} / c_{1}\right)^{k} k^{k / 2} \prod_{a} \tau^{\frac{1}{16} m_{a}^{2}} .
$$

Now we use this bound in (4.27) to deduce that

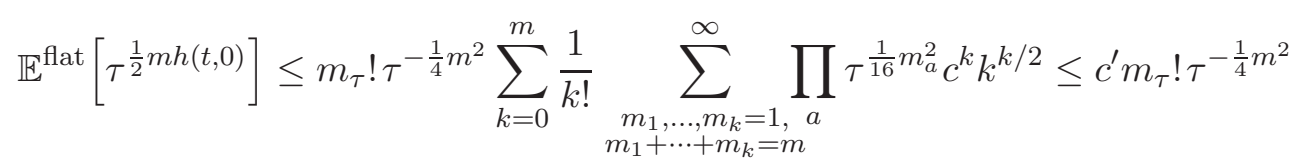

for some $c, c^{\prime}>0$ which are independent of $m$ (using Stirling's formula).

\section{Generating Function}

We are in position now to turn this into a formula for a certain generating function of flat ASEP. We will consider the following generalized $q$-exponential function: for fixed $\xi \in \mathbb{C}$ with $|\xi| \leq 1$,

$$
\exp _{q}(x ; \xi)=\sum_{k=0}^{\infty} \frac{1}{k_{q} !} \xi^{k(k-1)} x^{k} .
$$

Note that for $|\xi|<1$ and $x \in \mathbb{C}$ this is function is analytic in both variables. On the other hand, for fixed $\xi$ with $|\xi|=1$, this function is analytic in $x$ for $|x|<1$. Choosing $\xi$ to be 1 , $q^{1 / 2}$ and $q^{1 / 4}$ yields, respectively, $e_{q}(x), E_{q}(x)$ and $\exp _{q}(x)$ (although note that, for $\xi=1$ it only yields $e_{q}(x)$ restricted to $\left.|x|<1\right)$, see (2.5) and (2.6).

The next result gives a formula for the expectation of $\exp _{\tau}\left(\zeta \tau^{\frac{1}{2} h(t, 0)} ; \xi\right)$ for $|\xi| \leq \tau^{1 / 4}$. The restriction on $|\xi|$ comes from the fact that we will use our moment bound (4.28). 
Theorem 5.1. Let $\xi, \zeta \in \mathbb{C}$ and assume that either $|\xi|<\tau^{1 / 4}$ or $|\xi|=\tau^{1 / 4}$ and $|\zeta|<\tau^{1 / 4}$. Then

$$
\begin{aligned}
& \mathbb{E}^{\mathrm{flat}}\left[\exp _{\tau}\left(\zeta \tau^{\frac{1}{2} h(t, 0)} ; \xi\right)\right]=\sum_{k=0}^{\infty}(-1)^{k} \frac{1}{k !} \sum_{m_{1}, \ldots, m_{k}=1}^{\infty} \frac{1}{(2 \pi \mathrm{i})^{k}} \int_{\left(\mathbb{R}_{\geq 0}\right)^{k}} d \vec{\lambda} \\
& \times \zeta^{\sum_{a} m_{a}} \tau^{-\frac{1}{4}\left(\sum_{a} m_{a}\right)^{2}} \xi^{\left(\sum_{a} m_{a}\right)^{2}-\sum_{a} m_{a}} \operatorname{Pf}\left[K^{\mathrm{flat}}\left(\lambda_{a}, \lambda_{b} ; m_{a}, m_{b}\right)\right]_{a, b=1}^{k}
\end{aligned}
$$

with $K^{\text {flat }}$ as in (4.24). Moreover, the series on the right hand side is absolutely convergent.

Proof. Using the definition of $\exp _{\tau}(\cdot ; \xi)$ and interchanging sum and expectation we have

$$
\mathbb{E}^{\text {flat }}\left[\exp _{\tau}\left(\zeta \tau^{\frac{1}{2} h(t, 0)} ; \xi\right)\right]=\sum_{k=0}^{\infty} \frac{1}{k_{\tau} !} \zeta^{k} \xi^{k(k-1)} \mathbb{E}^{\text {flat }}\left[\tau^{\frac{1}{2} k h(t, 0)}\right] .
$$

Here, in order to justify the use of Fubini's Theorem, we are using Corollary 4.5, which gives $\mathbb{E}^{\text {flat }}\left[\tau^{\frac{1}{2} k h(t, 0)}\right] \leq c k_{\tau} ! \tau^{-k^{2} / 4}$, and implies that the double sum on the right hand side is absolutely summable under the stated conditions on $\xi$ and $\zeta$.

Now rewrite the right hand side of (4.27) as

$$
k_{\tau} ! \sum_{\ell=0}^{\infty} \frac{(-1)^{\ell}}{\ell !} \sum_{\substack{m_{1}, \ldots, m_{\ell}=1, m_{1}+\cdots+m_{\ell}=k}}^{\infty} \tau^{-\frac{1}{4}\left(\sum_{a} m_{a}\right)^{2}} Q(\vec{m})
$$

with $Q(\vec{m})=\int_{\left(\mathbb{R}_{\geq 0}\right)^{\ell}} d \vec{\lambda} \operatorname{Pf}\left[K^{\text {flat }}\left(\lambda_{a}, \lambda_{b} ; m_{a}, m_{b}\right)\right]_{a, b=1}^{\ell}$. To get a formula for the right hand side of (5.2) we multiply this by $\frac{1}{k_{\tau} !} \zeta^{k} \xi^{k(k-1)}$, sum over $k=0, \ldots, N$ and then take $N \rightarrow \infty$ to get

$$
\mathbb{E}^{\text {flat }}\left[\exp _{\tau}\left(\zeta \tau^{\frac{1}{2} h(t, 0)} ; \xi\right)\right]=\lim _{N \rightarrow \infty} \sum_{\ell=0}^{\infty} \frac{(-1)^{\ell}}{\ell !} \sum_{\substack{m_{1}, \ldots, m_{\ell}=1, m_{1}+\cdots+m_{\ell} \leq N}}^{\infty} \zeta^{\sum_{a} m_{a}} \tau^{-\frac{1}{4}\left(\sum_{a} m_{a}\right)^{2}} \xi^{\left(\sum_{a} m_{a}\right)^{2}-\sum_{a} m_{a}} Q(\vec{m}) .
$$

We claim that the limit can be taken inside the first sum, which yields

$$
\mathbb{E}^{\text {flat }}\left[\exp _{\tau}\left(\zeta \tau^{\frac{1}{2} h(t, 0)} ; \xi\right)\right]=\sum_{\ell=0}^{\infty} \frac{(-1)^{\ell}}{\ell !} \sum_{m_{1}, \ldots, m_{\ell}=1}^{\infty} \zeta^{\sum_{a} m_{a}} \tau^{-\frac{1}{4}\left(\sum_{a} m_{a}\right)^{2}} \xi^{\left(\sum_{a} m_{a}\right)^{2}-\sum_{a} m_{a}} Q(\vec{m})
$$

and proves the result. To see that the limit can be taken inside it is enough to show that the right hand side above is absolutely summable. But this follows from (4.29), which gives $|Q(\vec{m})| \leq c^{\ell} \ell^{\ell / 2} \tau^{\sum_{a} m_{a}^{2} / 16}$. In fact, using this bound we get

$$
\begin{aligned}
& \sum_{\ell=0}^{\infty} \frac{1}{\ell !} \sum_{m_{1}, \ldots, m_{\ell}=1}^{\infty}|\zeta|^{\sum_{a} m_{a}} \tau^{-\frac{1}{4}\left(\sum_{a} m_{a}\right)^{2}}|\xi|^{\left(\sum_{a} m_{a}\right)^{2}-\sum_{a} m_{a}}|Q(\vec{m})| \\
& \quad \leq \sum_{\ell=0}^{\infty} \frac{c^{\ell} \ell^{\ell / 2}}{\ell !} \sum_{m_{1}, \ldots, m_{\ell}=1}^{\infty}|\zeta|^{\sum_{a} m_{a}} \tau^{-\frac{1}{4}\left(\sum_{a} m_{a}\right)^{2}+\frac{1}{16} \sum_{a} m_{a}^{2}}|\xi|^{\left(\sum_{a} m_{a}\right)^{2}-\sum_{a} m_{a}}
\end{aligned}
$$

which is finite, as desired, by Stirling's formula and our assumptions on $\xi$ and $\zeta$.

We focus now on the case $\xi=\tau^{1 / 4}$ of Theorem 5.1, which yields the $\exp _{\tau}$-Laplace transform. In this case the factor $\xi^{\left(\sum_{a} m_{a}\right)^{2}}$ in front of the Pfaffian in (5.1) cancels exactly with $\tau^{-\left(\sum_{a} m_{a}\right)^{2} / 4}$, and hence the sums in $m_{a}$ can be brought inside the Pfaffian. To this end, and in view of the definition of $K^{\text {flat }}$ in (4.24), we define, for $\zeta \in \mathbb{C}$,

$$
\widetilde{K}^{\text {flat }, \zeta}\left(\lambda_{a}, \lambda_{b}\right)=\left[\begin{array}{cc}
\widetilde{K}_{1,1}^{\text {flat }, \zeta}\left(\lambda_{a}, \lambda_{b}\right) & \widetilde{K}_{1,2}^{\text {flat }, \zeta}\left(\lambda_{a}, \lambda_{b}\right) \\
-\widetilde{K}_{1,2}^{\text {flat }, \zeta}\left(\lambda_{b}, \lambda_{a}\right) & \widetilde{K}_{2,2}^{\text {flat }, \zeta}\left(\lambda_{a}, \lambda_{b}\right)
\end{array}\right]
$$


with

$$
\begin{aligned}
& \begin{aligned}
\widetilde{K}_{1,1}^{\text {flat }, \zeta}\left(\lambda_{a}, \lambda_{b}\right)= & \sum_{m=1}^{\infty} \frac{1}{\pi \mathrm{i}} f_{C_{0,1}} d y \tau^{\frac{1}{2} m^{2}} \zeta^{2 m} \mathfrak{v}\left(\lambda_{a}, y, m\right) \mathfrak{v}\left(\lambda_{b}, 1 / y, m\right) \mathfrak{u}_{\mathrm{ap}}(y, m) \\
& +\frac{1}{2} \sum_{m, m^{\prime}=1}^{\infty} \sum_{\sigma, \sigma^{\prime} \in\{-1,1\}}\left(-\sigma \sigma^{\prime}\right)^{m \wedge m^{\prime}+1} \operatorname{sgn}\left(\sigma^{\prime} m^{\prime}-\sigma m\right) \\
& \times \tau^{\frac{1}{4}\left(m^{2}+\left(m^{\prime}\right)^{2}\right)-\frac{1}{2}\left(m+m^{\prime}\right)} \zeta^{m+m^{\prime}} \mathfrak{v}\left(\lambda_{a}, \sigma, m\right) \mathfrak{v}\left(\lambda_{b}, \sigma^{\prime}, m^{\prime}\right),
\end{aligned} \\
& \widetilde{K}_{1,2}^{\text {flat }, \zeta}\left(\lambda_{a}, \lambda_{b}\right)=-\frac{1}{2} \sum_{m=1}^{\infty} \sum_{\sigma \in\{-1,1\}} \tau^{\frac{1}{4} m^{2}-\frac{1}{2} m} \zeta^{m} \mathfrak{v}\left(\lambda_{a}, \sigma, m\right), \\
& \widetilde{K}_{2,2}^{\text {flat }, \zeta}\left(\lambda_{a}, \lambda_{b}\right)= \\
& \frac{1}{2} \operatorname{sgn}\left(\lambda_{b}-\lambda_{a}\right),
\end{aligned}
$$

where $\mathfrak{u}_{\mathrm{ap}}$, and $\mathfrak{u}_{\mathrm{u}}$ are given in (4.7) and $\mathfrak{v}$ is given in (4.23). Then using Theorem 5.1 and Lemma B.3 we deduce the following

Theorem 5.2. For $|\zeta|<\tau^{1 / 4}$,

$$
\begin{aligned}
\mathbb{E}\left[\exp _{\tau}\left(\zeta \tau^{\frac{1}{2} h(t, 0)}\right)\right] & \left.=\sum_{k \geq 0} \frac{1}{k !} \int_{\left(\mathbb{R}_{\geq 0}\right)^{k}} d \vec{\lambda}(-1)^{k} \operatorname{Pf}\left[\widetilde{K}^{\text {flat }, \zeta}\left(\lambda_{a}, \lambda_{b}\right)\right]_{a, b=1}^{k}\right]_{a, b=1}^{k} \\
& =\operatorname{Pf}\left[J-\widetilde{K}^{\text {flat }, \zeta}\right]_{L^{2}([0, \infty))}
\end{aligned}
$$

Finally we are ready to prove Theorem 2.1, for which we need to check that the kernel given in (5.3) coincides with the one given in (2.12). The proof amounts to what can be considered as an ASEP version of the "Airy trick" commonly used in the physics literature. In fact, the representation of $\mathfrak{v}(\lambda, y, m)$ through an (inverse) double-sided Laplace transform (see (5.7) below) is an exact analog of the identity

$$
e^{m^{3} / 3}=\int_{-\infty}^{\infty} d w \operatorname{Ai}(w) e^{w m}
$$

for $m>0$ used in [LDC12] (see (42) and the identity right before (137) in that paper). A crucial difference, though, is that in [LDC12] (as in other situations were the "trick" is applied), the authors are dealing with a divergent series, and the Airy trick is used to resum it through an illegal interchange of sum and integration, whereas in our case such an interchange will be fully justified.

Proof of Theorem 2.1. For simplicity we will write $K$ instead of $\widetilde{K}^{\text {flat }, \zeta}$ throughout this proof. The only two pieces of the kernel for which we need to show that the two definitions coincide are $K_{1,1}$ and $K_{1,2}$, and we will begin with the latter. Throughout the rest of the proof we fix $\beta>0$ as in the Section 2.2.

For $\zeta \notin \mathbb{R}_{>0}$ define

$$
\psi(s, \lambda, y ; \zeta)=(-\zeta)^{s} \tau^{-\beta s^{2}-\frac{1}{4} s} \frac{\left(\tau y^{2} ; \tau\right)_{\infty}}{(1-\tau)^{s}\left(\tau^{1+s} y^{2} ; \tau\right)_{\infty}} \mathfrak{v}(\lambda, y, s)
$$

Recalling the definition of $\mathfrak{v}$ in (4.23), this definition coincides with the one given in (2.8). Moreover, for $m \in \mathbb{Z}_{\geq 1}$ and $\sigma \in\{-1,1\}$, since $\frac{(\tau ; \tau)_{\infty}}{(1-\tau)^{m}\left(\tau^{1+m} ; \tau\right)_{\infty}}=m_{\tau} !, \psi$ satisfies

$$
\tau^{\frac{1}{4} m^{2}-\frac{1}{2} m} \zeta^{m} \mathfrak{v}(\lambda, \sigma, m)=(-1)^{m} \frac{\tau^{\left(\frac{1}{4}+\beta\right) m^{2}-\frac{1}{4} m}}{m_{\tau} !} \psi(m, \lambda, \sigma ; \zeta)
$$


Therefore

$$
K_{1,2}\left(\lambda_{a}, \lambda_{b}\right)=-\frac{1}{2} \sum_{m=1}^{\infty} \sum_{\sigma \in\{-1,1\}}(-1)^{m} \frac{\tau^{\left(\frac{1}{4}+\beta\right) m^{2}-\frac{1}{4} m}}{m_{\tau} !} \psi\left(m, \lambda_{a}, \sigma ; \zeta\right) .
$$

Now we introduce the inverse double-sided Laplace transform of $\psi$ (in $s$ ), given by

$$
\check{\psi}(\omega, \lambda, y ; \zeta)=\frac{1}{2 \pi \mathrm{i}} \int_{\mathrm{i} \mathbb{R}} d s e^{s \omega} \psi(s, \lambda, y ; \zeta)
$$

This integral converges thanks to the factor $\tau^{-\beta s^{2}}$ which we introduced in $\psi$, and we have the inversion formula (see [Wid41], Theorem VI.19a)

$$
\psi(m, \lambda, y ; \zeta)=\int_{-\infty}^{\infty} d \omega e^{-m \omega} \check{\psi}(\omega, \lambda, y ; \zeta)
$$

Substituting (5.7) into the right hand side of (5.6) yields

$$
K_{1,2}\left(\lambda_{a}, \lambda_{b}\right)=-\frac{1}{2} \sum_{m=1}^{\infty} \sum_{\sigma \in\{-1,1\}} \frac{\tau^{\left(\frac{1}{4}+\beta\right) m^{2}-\frac{1}{4} m}}{m_{\tau} !} \int_{-\infty}^{\infty} d \omega e^{-m \omega} \check{\psi}\left(\omega, \lambda_{a}, \sigma ; \zeta\right) .
$$

In order to finish the verification of the identity for $K_{1,2}$ all that remains is to interchange the order of the summation in $m$ and the integral in $\omega$, which, as required in view of (2.9), (2.10) and (2.12), leads to

$$
K_{1,2}^{\mathrm{flat}}\left(\lambda_{a}, \lambda_{b}\right)=\frac{1}{2} \sum_{\sigma \in\{-1,1\}} \int_{-\infty}^{\infty} d \omega F_{3}\left(e^{-\omega}\right) \check{\psi}\left(\omega, \lambda_{a}, \sigma ; \zeta\right)
$$

with

$$
F_{3}(z)=-\sum_{m=1}^{\infty} \frac{\tau^{\left(\frac{1}{4}+\beta\right) m^{2}-\frac{1}{4} m}}{m_{\tau} !}(-z)^{m}
$$

The application of Fubini's theorem will be justified once we check that

$$
\int_{-\infty}^{\infty} d \omega \sum_{m=1}^{\infty}\left|\sigma \frac{\tau^{\left(\frac{1}{4}+\beta\right) m^{2}-\frac{1}{4} m}}{m_{\tau} !} e^{-m \omega} \check{\psi}(\omega, \lambda, \sigma ; \zeta)\right|<\infty .
$$

We first observe that $\psi(s, \lambda, \sigma ; \zeta)=\tau^{-\beta s^{2}} \zeta^{s} G\left(\tau^{s}, \lambda, \sigma\right)$, where $G$ is a continuous function, whose arguments vary over compact domains (recall that $s \in \mathrm{i} \mathbb{R}$ ). Therefore there is some $C>0$ such that

$$
|\psi(s, \lambda, \sigma ; \zeta)| \leq C \tau^{-\beta s^{2}}\left|\zeta^{s}\right|
$$

Let us write $a=\left(\frac{1}{4}+\beta\right)|\log (\tau)|$ and $b=\beta|\log (\tau)|$. Then we have, using the last inequality,

$$
|\check{\psi}(\omega, \lambda, \sigma ; \zeta)| \leq C \int_{\mathrm{i} \mathbb{R}} d s e^{b s^{2}+s \omega}=C \sqrt{\frac{\pi}{b}} e^{-\omega^{2} / 4 b}
$$

and thus the left hand side of (5.8) can be bounded by a constant multiple of

$$
\sqrt{\frac{\pi}{b}} \int_{-\infty}^{\infty} d \omega \sum_{m=1}^{\infty} e^{-a m^{2}-(1 / 4+\omega) m-\omega^{2} / 4 b} \leq C^{\prime} \frac{1}{\sqrt{a b}} \int_{-\infty}^{\infty} d \omega e^{(\omega+1 / 4)^{2} / 4 a-\omega^{2} / 4 b}<\infty
$$

for some $C^{\prime}>0$, where the last integral is finite since $0<b<a$. 
The argument for $K_{1,1}$ is similar. Using (5.5) and the definition of $\mathfrak{u}_{\text {ap }}$ in (4.7) we may write

$$
\begin{array}{rl}
K_{1,1}\left(\lambda_{a}, \lambda_{b}\right)=\sum_{m=1}^{\infty} \frac{1}{\pi \mathrm{i}} \int_{C_{0,1}} & d y(-1)^{m}(1-\tau)^{2 m} \tau^{\left(\frac{1}{2}+2 \beta\right) m^{2}-m} \frac{1+y^{2}}{y^{2}-1} \\
& \times \frac{\left(\tau^{1+m} y^{2} ; \tau\right)_{\infty}\left(\tau^{1+m} / y^{2} ; \tau\right)_{\infty}}{\left(\tau y^{2} ; \tau\right)_{\infty}\left(\tau / y^{2} ; \tau\right)_{\infty}} \psi\left(m, \lambda_{a}, y ; \zeta\right) \psi\left(m, \lambda_{b}, \frac{1}{y} ; \zeta\right) \\
+ & \frac{1}{2} \sum_{m, m^{\prime}=1}^{\infty} \sum_{\sigma, \sigma^{\prime} \in\{-1,1\}}(-1)^{m+m^{\prime}}\left(-\sigma \sigma^{\prime}\right)^{m \wedge m^{\prime}+1} \operatorname{sgn}\left(\sigma^{\prime} m^{\prime}-\sigma m\right) \\
& \times \frac{\tau^{\left(\frac{1}{4}+\beta\right)\left(m^{2}+\left(m^{\prime}\right)^{2}\right)-\frac{1}{4}\left(m+m^{\prime}\right)}}{m_{\tau} ! m_{\tau}^{\prime} !} \psi\left(m, \lambda_{a}, \sigma ; \zeta\right) \psi\left(m^{\prime}, \lambda_{b}, \sigma^{\prime} ; \zeta\right) .
\end{array}
$$

Plugging in the Laplace transform formula (5.7) for $\psi$, we would like to interchange the integration in $\omega$ and $\omega^{\prime}$ with the summation in $m$ and $m^{\prime}$ as in the $K_{1,2}$ case. This leads to

$$
\begin{aligned}
K_{1,1}\left(\lambda_{a}, \lambda_{b}\right)= & \int_{\mathbb{R}^{2}} d \omega d \omega^{\prime} \frac{1}{\pi i} \int_{C_{0,1}} d y \check{\psi}\left(\omega, \lambda_{a}, y ; \zeta\right) \check{\psi}\left(\omega^{\prime}, \lambda_{b}, \frac{1}{y} ; \zeta\right) F_{1}\left(e^{-\omega-\omega^{\prime}}, y\right) \\
& +\frac{1}{2} \sum_{\sigma, \sigma^{\prime} \in\{-1,1\}} \int_{\mathbb{R}^{2}} d \omega d \omega^{\prime} \check{\psi}\left(\omega, \lambda_{a}, \sigma ; \zeta\right) \check{\psi}\left(\omega^{\prime}, \lambda_{b}, \sigma^{\prime} ; \zeta\right) F_{2}\left(e^{-\omega}, e^{-\omega^{\prime}} ; \sigma, \sigma^{\prime}\right),
\end{aligned}
$$

with

$$
\begin{array}{r}
F_{1}(z, y)=\sum_{m=1}^{\infty}(-z)^{m}(1-\tau)^{2 m} \tau^{\left(\frac{1}{2}+2 \beta\right) m^{2}-m} \frac{1+y^{2}}{y^{2}-1} \frac{\left(\tau^{1+m} y^{2} ; \tau\right)_{\infty}\left(\tau^{1+m} / y^{2} ; \tau\right)_{\infty}}{\left(\tau y^{2} ; \tau\right)_{\infty}\left(\tau / y^{2} ; \tau\right)_{\infty}} \\
F_{2}\left(z_{1}, z_{2} ; \sigma_{1}, \sigma_{2}\right)=\sum_{m_{1}, m_{2}=1}^{\infty}\left(-z_{1}\right)^{m_{1}}\left(-z_{2}\right)^{m_{2}}\left(-\sigma_{1} \sigma_{2}\right)^{m_{1} \wedge m_{2}+1} \operatorname{sgn}\left(\sigma_{2} m_{2}-\sigma_{1} m_{1}\right) \\
\times \frac{\tau^{\left(\frac{1}{4}+\beta\right)\left(m_{1}^{2}+m_{2}^{2}\right)-\frac{1}{4}\left(m_{1}+m_{2}\right)}}{\left(m_{1}\right)_{\tau} !\left(m_{2}\right)_{\tau} !},
\end{array}
$$

which is exactly what we need. Hence all that remains is to justify the application of Fubini's Theorem. To this end, in the case of the first summand it is enough to check that

$$
\begin{aligned}
\int_{\mathbb{R}^{2}} d \omega_{1} d \omega_{2}\left|\frac{1}{2 \pi \mathrm{i}} \int_{C_{0,1}} d y \frac{1+y^{2}}{y^{2}-1} \check{\psi}\left(\omega_{1}, \lambda_{a}, y ; \zeta\right) \check{\psi}\left(\omega_{2}, \lambda_{b}, \frac{1}{y} ; \zeta\right)\right| \\
\times \sum_{m=1}^{\infty}\left|e^{-\left(\omega_{1}+\omega_{2}\right) m} \frac{\tau^{\left(\frac{1}{2}+2 \beta\right) m^{2}-m}}{\left(m_{\tau} !\right)^{2}}\right|<\infty
\end{aligned}
$$

The $y$ contour passes through singularities of the factor $\frac{1+y^{2}}{y^{2}-1}$ at $y= \pm 1$ and thus, as a principal value integral, it equals half the residues of the integrand at those points plus the same integral with the contour replaced by a circle $\widetilde{C}_{0,1}$ of radius slightly smaller than 1. Now the residues of $\frac{1+y^{2}}{y^{2}-1}$ at $y= \pm 1$ equal \pm 1 so the residues of the integrand at these points equal $\pm \check{\psi}\left(\omega_{1}, \lambda_{a}, \pm 1 ; \zeta\right) \check{\psi}\left(\omega_{2}, \lambda_{b}, \pm 1 ; \zeta\right)$. On the other hand, the estimate (5.9) still holds (with a different constant) with $\sigma$ on the left hand side replaced by $y$, uniformly in $y \in \widetilde{C}_{0,1} \cup\{-1,1\}$. Therefore

$$
\left|\frac{1}{2 \pi \mathrm{i}} \int_{C_{0,1}} d y \frac{1+y^{2}}{y^{2}-1} \check{\psi}\left(\omega_{1}, \lambda_{a}, y ; \zeta\right) \check{\psi}\left(\omega_{2}, \lambda_{b}, \frac{1}{y} ; \zeta\right)\right| \leq C e^{-\omega^{2} / 4 b}+C e^{-\omega^{2} / 4 b} \int_{\widetilde{C}_{0,1}} d y\left|\frac{1+y^{2}}{y^{2}-1}\right|
$$

where, we recall, $b=\beta|\log (\tau)|$ (and later $a=\left(\frac{1}{4}+\beta\right)|\log (\tau)|$ ). The last integral is finite, and thus this shows that the left hand side of (5.10) is bounded above by a constant multiple 
of

$$
\int_{\mathbb{R}^{2}} d \omega_{1} d \omega_{2} e^{-\left(\omega_{1}^{2}+\omega_{2}^{2}\right) / 4 b} \sum_{m=1}^{\infty} e^{-\left(\omega_{1}+\omega_{2}+1\right) m} \frac{e^{-2 a m^{2}}}{\left(m_{\tau} !\right)^{2}} \leq \int_{\mathbb{R}^{2}} d \omega_{1} d \omega_{2} e^{\left(\omega_{1}+\omega_{2}+1\right)^{2} / 8 a-\left(\omega_{1}^{2}+\omega_{2}^{2}\right) / 4 b},
$$

which as before is finite thanks to the fact that $a>b$. This yields (5.10) and finishes the justification of the desired identity for the first summand of $K_{1,1}$. The justification for the second summand is entirely analogous to the argument for $\widetilde{K}_{1,2}^{\text {flat }}$ above, and thus we omit it. This concludes out proof of the equality between the kernels given in (2.12) and (5.3).

\section{Moment formulas for flat KPZ/Stochastic heat EQUation}

In this section we will describe moment formulas for the solution of the KPZ equation with flat initial condition which are obtained in an analogous way as those for ASEP. Versions of these formulas already appeared in the physics literature in [LDC12].

The one-dimensional Kardar-Parisi-Zhang (KPZ) "equation" is given by

$$
\partial_{t} h=\frac{1}{2} \partial_{x}^{2} h-\frac{1}{2}\left[\left(\partial_{x} h\right)^{2}-\infty\right]+\xi .
$$

where $\xi$ is a space-time white noise. This SPDE is ill-posed as written but, at least on the torus, it can be made sense of by a renormalization procedure introduced by M. Hairer in [Hai13; Hai14]. His solutions coincide with the Cole-Hopf solution obtained by setting $h(t, x)=-\log Z(t, x)$, where $Z$ is the unique solution to the (well-posed) stochastic heat equation (SHE)

$$
\partial_{t} Z=\frac{1}{2} \partial_{x}^{2} Z+\xi Z
$$

The formulas which we will obtain will actually be for the moments $\mathbb{E}^{\text {flat }}\left[Z(t, 0)^{m}\right]$ of the SHE with flat initial data, which means

$$
Z(0, x)=1 \quad \text { or } \quad h(t, x)=0 .
$$

Of course, this means that we are getting formulas for the exponential moments $\mathbb{E}^{\text {flat }}\left[e^{-m h(t, 0)}\right]$ of flat KPZ. One can also think of this in terms of the solution of the delta Bose gas with flat initial data, which is the solution $v(t ; \vec{x})$ of the following system of equations, where we write $W_{k}=\left\{\vec{x} \in \mathbb{R}^{k}: x_{1}<x_{2}<\cdots<x_{k}\right\}$ (see [BC14; OQR14] for more details):

(1) For $\vec{x} \in W_{k}$,

$$
\partial_{t} v(t ; \vec{x})=\frac{1}{2} \Delta v(t ; \vec{x}),
$$

where the Laplacian acts on $\vec{x}$.

(2) For $\vec{x}$ on the boundary of $W_{k}$, with $x_{a}=x_{a+1}$,

$$
\left(\partial_{x_{a}}-\partial_{x_{a+1}}-1\right) v(t, \vec{x})=0 .
$$

(3) For $\vec{x} \in W_{k}$,

$$
\lim _{t \rightarrow 0} v(t ; \vec{x})=1
$$

It is widely accepted in the physics literature that if $Z(t, x)$ is a solution of the SHE, then $v(t ; \vec{x})=\mathbb{E}\left[Z\left(t, x_{1}\right) \cdots Z\left(t, x_{k}\right)\right]$ is a solution of the delta Bose gas. This fact is proved in [MFQR15], where it is also shown that there is at most one solution.

In [OQR14] we obtained an explicit formula for the moments of the SHE started with half-flat initial condition by taking a weakly asymmetric limit of the half-flat ASEP moment formula (Theorem 2.4 above). The same formula was obtained in [OQR14] directly for the delta Bose gas with a slightly more general initial condition using the same method which led in that paper to the moment formulas for half-flat ASEP. 
Proposition 6.1. For $\theta>0$ define (with $x$ appearing $k$ times in the argument)

$$
v_{\theta}^{\mathrm{h}-\mathrm{fl}}(t ; x, \ldots, x)=2^{k} k ! \sum_{\ell=0}^{k} \frac{1}{\ell !} \sum_{\substack{n_{1}, \ldots, n_{\ell} \geq 1 \\ n_{1}+\cdots+n_{\ell}=k}} \frac{1}{(2 \pi \mathrm{i})^{\ell}} \int_{(\alpha+\mathrm{i} \mathbb{R})^{k}} d \vec{w} I_{\theta}(\vec{w} ; \vec{n})
$$

with $\alpha>0$ and with $I_{\theta}$ given by

$$
\begin{aligned}
& I_{\theta}(\vec{w}, \vec{n})=2^{k} \prod_{a} \frac{\Gamma\left(2 w_{a}\right)}{n_{a} \Gamma\left(2 w_{a}+n_{a}\right)} e^{t\left[\frac{1}{12} n_{a}^{3}-\frac{1}{12} n_{a}+n_{a}\left(w_{a}-\theta\right)^{2}\right]+x n_{a}\left(w_{a}-\theta\right)} \\
& \times \prod_{a<b} \frac{\Gamma\left(w_{a}+w_{b}+\frac{1}{2}\left(n_{a}-n_{b}\right)\right) \Gamma\left(w_{a}+w_{b}-\frac{1}{2}\left(n_{a}-n_{b}\right)\right)}{\Gamma\left(w_{a}+w_{b}-\frac{1}{2}\left(n_{a}+n_{b}\right)\right) \Gamma\left(w_{a}+w_{b}+\frac{1}{2}\left(n_{a}+n_{b}\right)\right)} \\
& \times \frac{\left(w_{a}-w_{b}+\frac{1}{2}\left(n_{a}-n_{b}\right)\right)\left(w_{a}-w_{b}-\frac{1}{2}\left(n_{a}-n_{b}\right)\right)}{\left(w_{a}-w_{b}\right)\left(w_{a}-w_{b}+\frac{1}{2}\left(n_{a}+n_{b}\right)\right)} .
\end{aligned}
$$

Then the solution $v(t ; \vec{x})$ of the delta Bose gas with the initial data in (3) replaced by the tilted half-flat initial condition $\lim _{t \rightarrow 0} v(t ; \vec{x})=\prod_{a} e^{-\theta x_{a}} \mathbf{1}_{x_{a} \geq 0}$ coincides with $v_{\theta}^{\mathrm{h}-\mathrm{fl}}(t ; x, \ldots, x)$ when $\vec{x}=(x, \ldots, x)$ (with $x \in \mathbb{R}$ repeated $k$ times). Moreover, if $Z(t, x)$ is the solution of the SHE (6.1) with half-flat initial condition $Z(0, x)=\mathbf{1}_{x \geq 0}$, then (denoting by $\mathbb{E}^{\mathrm{h}-\mathrm{fl}}$ the expectation with this initial condition)

$$
\mathbb{E}^{\mathrm{h}-\mathrm{f}}\left[Z(t, x)^{k}\right]=v_{0}^{\mathrm{h}-\mathrm{fl}}(t ; x, \ldots, x) .
$$

This corresponds to Proposition 5.3 of [OQR14]. As in the ASEP case, we have used the Cauchy determinant identity (2.24) to expand the determinant which appears in that formula.

Just as (6.4) was obtained in [OQR14] as a weakly asymmetric limit of (2.21), one can take a weakly asymmetric limit of (4.27) to derive a moment formula for the SHE with flat initial condition $Z(0, x)=1$. Alternatively, one can take the $x \rightarrow \infty$ limit directly at the level of (6.2)-(6.4) to obtain such a formula. In fact, by statistical translation invariance of the white noise, $Z(t, x)$ with half-flat initial condition $Z(0, y)=\mathbf{1}_{y \geq 0}$ has the same distribution as $Z(t, 0)$ with initial condition $Z(0, y)=\mathbf{1}_{y \geq-x}$, and thus computing the $x \rightarrow \infty$ limit of $(6.2)$ leads to the moments of $Z(t, 0)$ with flat initial condition. This is the approach that we will take here (although we will not provide all the details). As for the case of ASEP, the calculations involved in the computation of this limit are quite involved, but they are completely analogous to those of Sections 3 and 4, as we describe next.

Note that the dependence on $x$ of the right hand side of (6.3) is only through factors of the form $e^{x w_{a} n_{a}}$, which suggests that we should deform the $w_{a}$ contours to a region where $\Re\left(w_{a}\right)<0$. More precisely, we will shift the $w_{a}$ contours to $-\delta+\mathrm{i} \mathbb{R}$ for some small $\delta>0$. The contour deformation involves crossing many poles, and a careful analysis shows that the pole structure is entirely analogous to the one described in Section 3.1 for ASEP. Now the unpaired poles occur at $w_{a}=0$, coming from the factor $\Gamma\left(2 w_{a}\right)$, while the paired poles occur at $w_{a}=-w_{b}$ in the case $n_{a}=n_{b}$, coming from Gamma factors in the numerator of the second line of (6.3). Crucially, as in the ASEP computation (though in a slightly simpler way), the dependence on $x$ is gone in all the residues that one has to compute as the contours are deformed. Since the free variables will now be integrated on a contour $-\delta+\mathrm{i} \mathbb{R}$ with $\delta>0$, these factors will vanish as $x \rightarrow \infty$, and as a result the limiting formula will be written only in terms of unpaired and paired variables, analogously to what was done in Section 3 for ASEP. Instead of going through the whole argument again, we will only state the result. Introduce the index set

$$
\bar{\Lambda}_{k_{1}, k_{2}}^{m}=\left\{\left(\vec{n}^{\mathrm{u}}, \vec{n}^{\mathrm{p}}\right) \in \mathbb{Z}_{\geq 1}^{k_{1}} \times \mathbb{Z}_{\geq 1}^{k_{2}}: \sum_{a=1}^{k_{1}} n_{a}^{\mathrm{u}}+2 \sum_{a=1}^{k_{2}} n_{a}^{\mathrm{p}}=m\right\} .
$$


Then

$$
\mathbb{E}^{\mathrm{flat}}\left[Z(t, 0)^{m}\right]=2^{m} m ! \sum_{k=0}^{m} \bar{\nu}_{k, m}^{\mathrm{flat}}(t)
$$

with

$$
\bar{\nu}_{k, m}^{\text {flat }}(t)=\sum_{\substack{k_{\mathrm{u}}, k_{\mathrm{p}} \geq 0 \\ k_{\mathrm{u}}+2 k_{\mathrm{p}}=k}} \frac{1}{k_{\mathrm{u}} ! 2^{k_{\mathrm{p}}} k_{\mathrm{p}} !} \overline{\mathfrak{J}}_{m}^{\text {flat }}\left(k_{\mathrm{u}}, k_{\mathrm{p}}\right),
$$

where

$$
\begin{aligned}
& \overline{\mathfrak{Z}}_{m}^{\text {flat }}\left(k_{\mathrm{u}}, k_{\mathrm{p}}\right)=\sum_{\left(\vec{n}^{\mathrm{u}}, \vec{n}^{\mathrm{p}}\right) \in \bar{\Lambda}_{k_{\mathrm{u}}, k_{\mathrm{p}}}^{m}} \frac{1}{(2 \pi \mathrm{i})^{k_{\mathrm{p}}}} \int_{(\mathrm{iR})^{k_{\mathrm{p}}}} d \vec{z}^{\mathrm{p}} \prod_{a \leq k_{\mathrm{p}}, b \leq k_{\mathrm{u}}} \frac{\left(n_{a}^{\mathrm{p}}-n_{b}^{\mathrm{u}}\right)^{2}-4\left(z_{a}^{\mathrm{p}}\right)^{2}}{\left(n_{a}^{\mathrm{p}}+n_{b}^{\mathrm{u}}\right)^{2}-4\left(z_{a}^{\mathrm{p}}\right)^{2}} \\
& \times \prod_{a=1}^{k_{\mathrm{p}}} \prod_{j=1}^{n_{a}^{\mathrm{p}}} \frac{1}{\left(z_{a}^{\mathrm{p}}\right)^{2}-j^{2}} \prod_{1 \leq a<b \leq k_{\mathrm{p}}} \frac{\left(n_{a}^{\mathrm{p}}-n_{b}^{\mathrm{p}}\right)^{2}-4\left(z_{a}^{\mathrm{p}}-z_{b}^{\mathrm{p}}\right)^{2}}{\left(n_{a}^{\mathrm{p}}+n_{b}^{\mathrm{p}}\right)^{2}-4\left(z_{a}^{\mathrm{p}}-z_{b}^{\mathrm{p}}\right)^{2}} \frac{\left(n_{a}^{\mathrm{p}}-n_{b}^{\mathrm{p}}\right)^{2}-4\left(z_{a}^{\mathrm{p}}+z_{b}^{\mathrm{p}}\right)^{2}}{\left(n_{a}^{\mathrm{p}}+n_{b}^{\mathrm{p}}\right)^{2}-4\left(z_{a}^{\mathrm{p}}+z_{b}^{\mathrm{p}}\right)^{2}} \\
& \times \frac{1}{2^{k_{\mathrm{u}}}} \prod_{a=1}^{k_{\mathrm{u}}} \frac{1}{n_{a}^{\mathrm{u} !}} e^{\frac{1}{12} t\left(\left(n_{a}^{\mathrm{u}}\right)^{3}-n_{a}^{\mathrm{u}}\right)} \prod_{a=1}^{k_{\mathrm{p}}} \frac{1}{n_{a}^{\mathrm{p}}} e^{t\left[\frac{1}{6}\left(n_{a}^{\mathrm{p}}\right)^{3}-\frac{1}{6} n_{a}^{\mathrm{p}}+2 n_{a}^{\mathrm{p}}\left(z_{a}^{\mathrm{p}}\right)^{2}\right]} \prod_{1 \leq a<b \leq k_{\mathrm{u}}}(-1)^{n_{a}^{\mathrm{u}} \wedge n_{b}^{\mathrm{u}}} \frac{\left|n_{a}^{\mathrm{u}}-n_{b}^{\mathrm{u}}\right|}{n_{a}^{\mathrm{u}}+n_{b}^{\mathrm{u}}} .
\end{aligned}
$$

This formula should be compared with the moment formula for flat ASEP provided in Theorem 3.6 (in fact, (6.5) can be obtained as a weakly asymmetric limit of that formula). Note that in the above discussion the $z_{a}^{\mathrm{p}}$ contours where deformed to $-\delta+i \mathbb{R}$, but here they have been replaced by $i \mathbb{R}$; we may do this here (i.e. take $\delta \rightarrow 0$ ) because the resulting integrand has no poles on $z_{a} \in \mathrm{i} \mathbb{R}$ (this analogous to what was done for ASEP, see the paragraph following (3.20)).

It is not hard to check that (6.6) corresponds to (108) in [LDC12] (with $k_{\mathrm{u}}=M$, $k_{\mathrm{p}}=N$ and setting $s=0$ in their formula, which comes from a scaling factor which we have not included at this point; note also that in [LDC12] the replacement $t=4 \lambda^{3}$ has been performed). Their heuristic derivation essentially consists in studying the poles which arise from taking $\theta \rightarrow 0$ at the same time as $x \rightarrow \infty$ directly in (6.2) with $\alpha=\theta$. Although their procedure is different and not fully justified, the algebraic structure is similar. In terms of (6.2), they start by shifting all the $w_{a}$ variables by $\theta$, which results in the contours turning into $i \mathbb{R}$. As they take $\theta \rightarrow 0$, some of the factors in the integrand now pass through singularities, which give rise to residues which are in correspondence with the ones arising from our paired and unpaired poles. Then they argue that taking $x \rightarrow \infty$ at the same time as $\theta \rightarrow 0$ results in only these types of terms remain in the limit, leading to the formula.

The final step is to find a Pfaffian representation for the moment formula (6.5). This is done again in an entirely analogous fashion to what was done for ASEP in Sections 4.1 and 4.2 (alternatively, and as we mentioned, one can take a weakly asymmetric limit of the moment formula in Proposition 2.3, which leads to the same expression). Let us skip the details and just write the result:

$$
\mathbb{E}^{\text {flat }}\left[Z(t, 0)^{m}\right]=m ! \sum_{k=0}^{m} \frac{(-1)^{k}}{k !} \sum_{\substack{m_{1}, \ldots, m_{k}=1 \\ m_{1}+\cdots+m_{k}=m}}^{\infty} \int_{\left(\mathbb{R}_{\geq 0}\right)^{k}} d \vec{\lambda} \operatorname{Pf}\left[\bar{K}\left(\lambda_{a}, \lambda_{b} ; m_{a}, m_{b}\right)\right]_{a, b=1}^{k}
$$


with $\bar{K}\left(\lambda_{1}, \lambda_{2} ; m_{1}, m_{2}\right)=\left[\begin{array}{cc}\bar{K}_{1,1}\left(\lambda_{1}, \lambda_{2} ; m_{1}, m_{2}\right) & \bar{K}_{1,2}\left(\lambda_{1}, \lambda_{2} ; m_{1}, m_{2}\right) \\ -\bar{K}_{1,2}\left(\lambda_{2}, \lambda_{1} ; m_{2}, m_{1}\right) & \bar{K}_{2,2}\left(\lambda_{1}, \lambda_{2} ; m_{1}, m_{2}\right)\end{array}\right]$ and

$$
\begin{aligned}
& \bar{K}_{1,1}\left(\lambda_{1}, \lambda_{2} ; m_{1}, m_{2}\right)= \mathbf{1}_{m_{1}=m_{2}}(-1)^{m_{1}+1} \frac{1}{8 \pi \mathrm{i}} f_{\mathrm{i} \mathbb{R}} d y \frac{1}{y} \frac{\Gamma(2 y)}{\Gamma(m+2 y)} \frac{\Gamma(-2 y)}{\Gamma(m-2 y)} \\
& \times e^{\left[\frac{1}{96} m_{1}^{3}+\frac{1}{8} m_{1} y^{2}\right] t-\frac{1}{4}\left(m_{1}-2 y\right) \lambda_{1}-\frac{1}{4}\left(m_{1}+2 y\right) \lambda_{2}} \\
&+\frac{1}{32} \frac{1}{\left(m_{1}-1\right) !} \frac{1}{\left(m_{2}-1\right) !}(-1)^{m_{1} \wedge m_{2}} \operatorname{sgn}\left(m_{1}-m_{2}\right) e^{\frac{1}{192}\left(m_{1}^{3}+m_{2}^{3}\right) t-\frac{1}{4}\left(m_{1} \lambda_{1}+m_{2} \lambda_{2}\right)}
\end{aligned}
$$

$\bar{K}_{1,2}\left(\lambda_{1}, \lambda_{2} ; m_{1}, m_{2}\right)=-\frac{1}{8} \frac{1}{\left(m_{1}-1\right) !} e^{\frac{1}{192} m_{1}^{3} t-\frac{1}{4} m_{1} \lambda_{1}}$

$\bar{K}_{2,2}\left(\lambda_{1}, \lambda_{2} ; m_{1}, m_{2}\right)=\frac{1}{2} \operatorname{sgn}\left(\lambda_{2}-\lambda_{1}\right)$.

This formula should be compared with the the $m$-th term of the (divergent) series written for $\sum_{m \geq 0} \frac{\zeta^{n}}{n !} \mathbb{E}^{\text {flat }}\left[Z(t, 0)^{m}\right]$ (for a specific choice of $\zeta$ ) in $(147)$ of $[\mathrm{LDC} 12]^{5}$.

\section{Appendix A. Formal GOE asymptotics for flat ASEP}

In this section we will provide a non-rigorous argument that shows that, under the scaling specified in (2.13), the right hand side of (2.11) recovers the GOE Tracy-Widom distribution.

More precisely, we perform a formal asymptotic analysis of $\operatorname{Pf}\left[J-\widetilde{K}^{f l a t, \zeta}\right]_{L^{2}([0, \infty))}$ with $\zeta=-\tau^{-t / 4+t^{1 / 3} r / 2}$ as $t \rightarrow \infty$ and obtain $\operatorname{Pf}\left[J-K_{r}\right]_{L^{2}([0, \infty))}$, with $K_{r}$ defined as in (2.15). This is the content of Section A.2, and should be interpreted as evidence for the conjecture

$$
\lim _{t \rightarrow \infty} \mathbb{P}^{\text {flat }}\left(\frac{h\left(t /(q-p), t^{2 / 3} x\right)-\frac{1}{2} t}{t^{1 / 3}} \geq-r\right)=\operatorname{Pf}\left[J-K_{r}\right]_{L^{2}([0, \infty))} .
$$

The issues discussed in Remark 2.2.vii preclude directly making this part of the asymptotics rigorous. However, even the formal critical point analysis has a complicated algebraic structure which needs to be explained. We will then show, rigorously, in Section A.3, that the right hand side recovers the Tracy-Widom GOE distribution:

$$
\operatorname{Pf}\left[J-K_{r}\right]_{L^{2}([0, \infty))}=F_{\mathrm{GOE}}(r) .
$$

A.1. Mellin-Barnes representation. There are two alternative ways to derive (formally) the $t \rightarrow \infty$ asymptotics of our formula. The first one consists in starting directly with the formula given in Theorem 2.1, which is written in a form that lends itself readily to critical point analysis (this is thanks to the rewriting of (5.3) through (5.7), which is analogous to the physicists' Airy trick, see the discussion in page 32 preceding the proof of the theorem). The difficulty with this approach is that it involves delicate asymptotics of the functions $F_{1}, F_{2}$ and $F_{3}$ (defined in $(2.10)$ ) which are as poorly behaved as $\exp _{\tau}$.

A different argument relies on using a Mellin-Barnes representation (see [BC14], which introduced this idea in this setting) for the sums (in $m$ and $m^{\prime}$ ) which appear in (5.3). The advantage of this approach is that the critical point analysis turns out to be relatively simple and one is not faced with the asymptotics of the functions $F_{1}, F_{2}$ and $F_{3}$. The

\footnotetext{
${ }^{5}$ There are two main differences between (6.7) and the $n$-th term of the formula written in [LDC12], besides some simple scaling and renaming of variables. First, in their formula they have applied the Airy trick (5.4) in order to rewrite expressions involving $e^{\frac{1}{192} m^{3} t}$ in terms of integrals of Airy functions. This can be done for (6.7) without any trouble (we have not done it because it leads to more complicated formulas). Second, one can check that obtaining their formula from ours involves (formally) integrating $\bar{K}_{1,1}$ in $\lambda_{1}$ and $\lambda_{2}$, integrating $\bar{K}_{1,1}$ in $\lambda_{1}$ and differentiating it in $\lambda_{2}$, and differentiating $\bar{K}_{2,2}$ in both $\lambda_{1}$ and $\lambda_{2}$. This can be justified (formally) using Lemma B.3 (twice). This difference stems from the delta Bose gas analog of the step performed at the ASEP level after (4.19), which is not done in [LDC12].
} 
difficulty, on the other hand, is that implementing the Mellin-Barnes representation turns out to be much harder than usual, due to the existence of a large number of additional poles (on top of the ones poles at the integers which are inherent in the representation) which have to be accounted for. The fact that all these additional poles end up cancelling is far from trivial and serves as another indication of the remarkable structure behind the flat ASEP formulas. Partly because of this reason, this is the approach we will follow presently.

In order to derive the Mellin-Barnes representation of the kernel $\widetilde{K}^{\text {flat }, \zeta}$ in (5.3) we will need to use the following generalization of the Mellin-Barnes representation formula which appears e.g. in [BC14]:

Lemma A.1. Let $C_{1,2, \ldots}$ be a negatively oriented contour enclosing all positive integers (e.g. $C_{1,2, \ldots}=\frac{1}{2}+\mathrm{i} \mathbb{R}$ oriented with increasing imaginary part). Let $g$ be a meromorphic function and let $\mathbb{A}$ be the set of all poles of $g$ lying to the right of $C_{1,2 \ldots}$. Assume that $\mathbb{A} \cap \mathbb{Z}_{\geq 1}=\emptyset$. Then for $\zeta \in \mathbb{C} \backslash \mathbb{R}_{>0}$ with $|\zeta|<1$ we have

$$
\sum_{n=1}^{\infty} g(n) \zeta^{n}=\frac{1}{2 \pi \mathrm{i}} \int_{C_{1,2, \ldots}} d s \frac{\pi}{\sin (-\pi s)}(-\zeta)^{s} g(s)-\sum_{w \in \mathbb{A}} \frac{\pi}{\sin (-\pi w)}(-\zeta)^{w} \operatorname{Res}_{s=w} g\left(\tau^{s}\right)
$$

provided that the left hand side converges and that there exist closed contours $C_{k}, k \in \mathbb{N}$ enclosing the positive integers from 1 to $k$ and such that the integral of the integrand on the right hand side over the symmetric difference of $C_{1,2, \ldots}$ and $C_{k}$ goes to zero as $k \rightarrow \infty$.

The proof is elementary and only uses the fact that $\pi / \sin (-\pi s)$ has poles at each $s=$ $k \in \mathbb{Z}$ with residue equal to $(-1)^{k+1}$. The (trivial) novelty of this formula is the appearance of a second family of poles at $\mathbb{A}$.

In order to apply this result to our formula we need to find a different expression for $\widetilde{K}_{1,1}^{\text {flat }, \zeta}$, which as written contains factors of the form $\mathbf{1}_{m_{a}=m_{b}},(-1)^{m_{a} \wedge m_{b}}$ and $(-1)^{m_{a}}$ (appearing inside the definition of $\mathfrak{u}_{\mathrm{ap}}\left(y, m_{a}\right)$ ) which are not suitable for a representation in terms of contour integrals. The following result can be proved in a similar way to Lemma 3.1:

Lemma A.2. Given any $m_{1} \neq m_{2} \in \mathbb{Z}$ with the same sign,

$$
\lim _{\eta \rightarrow 0} \frac{\Gamma\left(\frac{1}{2}\left(m_{1}-m_{2}\right)+\eta\right) \Gamma\left(\frac{1}{2}\left(m_{2}-m_{1}\right)+\eta\right)}{\Gamma\left(\frac{1}{2}\left(m_{1}+m_{2}\right)+\eta\right) \Gamma\left(-\frac{1}{2}\left(m_{1}+m_{2}\right)+\eta\right)} \frac{m_{2}-m_{1}+2 \eta}{m_{1}+m_{2}+2 \eta}=(-1)^{m_{1} \wedge m_{2}} \operatorname{sgn}\left(m_{2}-m_{1}\right) .
$$

(One can choose slightly different versions of the left hand side, but this form will turn out to be convenient later). As a consequence, if we define

$\mathfrak{h}_{\eta}\left(\sigma_{1}, \sigma_{2} ; m_{1}, m_{2}\right)= \begin{cases}\sigma_{2} \frac{\Gamma\left(\frac{1}{2}\left(m_{1}-m_{2}\right)+\eta\right) \Gamma\left(\frac{1}{2}\left(m_{2}-m_{1}\right)+\eta\right)}{\Gamma\left(\frac{1}{2}\left(m_{1}+m_{2}\right)+\eta\right) \Gamma\left(-\frac{1}{2}\left(m_{1}+m_{2}\right)+\eta\right)} \frac{m_{2}-m_{1}+2 \eta}{m_{1}+m_{2}+2 \eta} & \text { if } \sigma_{1}=\sigma_{2} \\ \sigma_{2} & \text { if } \sigma_{1} \neq \sigma_{2},\end{cases}$

then we may replace every factor of the form $\left(-\sigma_{a} \sigma_{b}\right)^{m_{a} \wedge m_{b}} \operatorname{sgn}\left(\sigma_{b} m_{b}-\sigma_{a} m_{a}\right)$ in our formula by $\lim _{\eta \rightarrow 0} \mathfrak{h}_{\eta}\left(\sigma_{a}, \sigma_{b} ; m_{a}, m_{b}\right)$. Something similar can be done for the product of $\mathbf{1}_{m_{a}=m_{b}}$ and the factor $(-1)^{m_{a}}$ appearing in $\mathfrak{u}_{\mathrm{ap}}\left(y_{a}, m_{a}\right)$, replacing it by $\lim _{\eta \rightarrow 0} \mathfrak{s}_{\eta}\left(m_{a}, m_{b}\right)$ with

$$
\mathfrak{s}_{\eta}\left(m_{1}, m_{2}\right)=\sin \left(\frac{1}{2} \pi\left(2 m_{1}+1\right)\right) \frac{\sin \left(\pi\left(m_{1}-m_{2}+\eta\right)\right)}{\pi\left(m_{1}-m_{2}+\eta\right)} .
$$

As we will see in the next result, the $\eta \rightarrow 0$ limit can be taken outside the sums and integrals. We state it at the level of the formula for the $\exp _{\tau}(x ; \xi)$ transform: 
Proposition A.3. For every $\xi, \zeta \in \mathbb{C}$ with $|\xi|<\tau^{1 / 4}$ or $|\xi|=\tau^{1 / 4}$ and $|\zeta|<\tau^{1 / 4}$ we have

$$
\begin{aligned}
\mathbb{E}^{\mathrm{flat}}\left[\exp _{\tau}\left(\zeta \tau^{\frac{1}{2} h(t, 0)} ; \xi\right)\right]=\lim _{\eta \rightarrow 0} \sum_{k=0}^{\infty}(-1)^{k} \frac{1}{k !} \sum_{m_{1}, \ldots, m_{k}=1}^{\infty} \int_{\left(\mathbb{R}_{\geq 0}\right)^{k}} d \vec{\lambda} \\
\times \zeta^{\sum_{a} m_{a}} \tau^{-\frac{1}{4}\left(\sum_{a} m_{a}\right)^{2}} \xi^{\left(\sum_{a} m_{a}\right)^{2}-\sum_{a} m_{a}} \operatorname{Pf}\left[K^{\mathrm{flat}, \eta}\left(\lambda_{a}, \lambda_{b} ; m_{a}, m_{b}\right)\right]_{a, b=1}^{k}
\end{aligned}
$$

where $K^{\text {flat, } \eta}$ is the $2 \times 2$ skew-symmetric matrix kernel defined by

$$
\begin{gathered}
K_{1,1}^{\mathrm{flat}, \eta}\left(\lambda_{a}, \lambda_{b} ; m_{a}, m_{b}\right)=\mathfrak{s}_{\eta}\left(m_{a}, m_{b}\right) \frac{1}{\pi \mathrm{i}} f_{C_{0,1}} d y \tau^{\frac{1}{2} m_{a}^{2}} \mathfrak{v}\left(\lambda_{a}, y, m_{a}\right) \mathfrak{v}\left(\lambda_{b}, \frac{1}{y}, m_{a}\right) \tilde{\mathfrak{u}}_{\mathrm{ap}}\left(y, m_{a}\right) \\
+\frac{1}{2} \sum_{\sigma, \sigma^{\prime} \in\{-1,1\}} \mathfrak{h}_{\eta}\left(\sigma, \sigma^{\prime} ; m_{a}, m_{b}\right) \tau^{\frac{1}{4}\left(m_{a}^{2}+m_{b}^{2}\right)} \mathfrak{v}\left(\lambda_{a}, \sigma, m_{a}\right) \mathfrak{v}\left(\lambda_{b}, \sigma^{\prime}, m_{b}\right) \mathfrak{u}_{\mathrm{u}}\left(\sigma, m_{a}\right) \mathfrak{u}_{\mathrm{u}}\left(\sigma^{\prime}, m_{b}\right), \\
K_{1,2}^{\mathrm{flat}, \eta}=K_{1,2}^{\mathrm{flat}}, \quad \text { and } \quad K_{2,2}^{\text {flat }, \eta}=K_{2,2}^{\text {flat }},
\end{gathered}
$$

where

$$
\tilde{\mathfrak{u}}_{\mathrm{ap}}(y, m)=(-1)^{m} \mathfrak{u}_{\mathrm{ap}}(y, m)=\tau^{-\frac{1}{2} n} \frac{1+y^{2}}{y^{2}-1}
$$

and $\mathfrak{u}_{\mathrm{u}}$ and $\mathfrak{v}$ were defined respectively in (4.7) and (4.23).

Proof. Consider the kernel $K^{\text {flat,0 }}$ defined as $\lim _{\eta \rightarrow 0} K^{\text {flat, } \eta}$. Then it is straightforward to check from Theorem 5.1, using Lemma A.2 and the fact that $\mathfrak{s}_{\eta}\left(m_{1}, m_{2}\right) \longrightarrow(-1)^{m_{1}} \mathbf{1}_{m_{1}=m_{2}}$ as $\eta \rightarrow 0$ for $m_{1}, m_{2} \in \mathbb{Z}$, that

$$
\begin{aligned}
\mathbb{E}^{\text {flat }}\left[\exp _{\tau}\left(\zeta \tau^{\frac{1}{2} h(t, 0)} ; \xi\right)\right]=\sum_{k=0}^{\infty}(-1)^{k} \frac{1}{k !} \sum_{m_{1}, \ldots, m_{k}=1}^{\infty} \frac{1}{(2 \pi \mathrm{i})^{k}} \int_{\left(\mathbb{R}_{\geq 0}\right)^{k}} d \vec{\lambda} \\
\times \zeta^{\sum_{a} m_{a}} \tau^{-\frac{1}{4}\left(\sum_{a} m_{a}\right)^{2}} \xi^{\left(\sum_{a} m_{a}\right)^{2}-\sum_{a} m_{a}} \operatorname{Pf}\left[K^{\text {flat }, 0}\left(\lambda_{a}, \lambda_{b} ; m_{a}, m_{b}\right)\right]_{a, b=1}^{k}
\end{aligned}
$$

To justify taking the $\eta \rightarrow 0$ limit outside use the Dominated Convergence Theorem together with an argument similar to the one used in the proof of Theorem 5.1 to show that the series on the right hand side of (A.1) is absolutely summable.

Note that, since $\mathfrak{s}_{\eta}\left(m_{a}, m_{b}\right)$ is replacing the factor $(-1)^{m_{a}} \mathbf{1}_{m_{a}=m_{b}}$, the first term of $K_{1,1}^{\text {flat, } \eta}$ in Proposition A.3 can be rewritten as

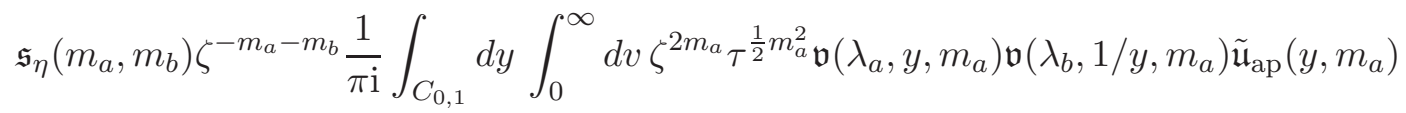

(as can be checked directly in the proof). Using this replacement, setting $\xi=\tau^{1 / 4}$ in Proposition A.3 and bringing the sums in $m_{a}$ inside the Pfaffian as in Section 5 yields the following formula:

$$
\mathbb{E}^{\text {flat }}\left[\exp _{\tau}\left(\zeta \tau^{\frac{1}{2} h(t, 0)}\right)\right]=\lim _{\eta \rightarrow 0} \operatorname{Pf}\left[J-\widetilde{K}^{\eta}\right]
$$


with (we omit here the dependence of the kernel on $\zeta$ )

$$
\begin{aligned}
& \widetilde{K}_{1,1}^{\eta}\left(\lambda, \lambda^{\prime}\right)=\sum_{m, m^{\prime}=1}^{\infty} \mathfrak{s}_{\eta}\left(m, m^{\prime}\right) \zeta^{-m-m^{\prime}} \frac{1}{\pi \mathrm{i}} f_{C_{0,1}} d y \zeta^{2 m} \tau^{\frac{1}{2} m^{2}} \mathfrak{v}(\lambda, y, m) \mathfrak{v}\left(\lambda^{\prime}, \frac{1}{y}, m\right) \tilde{\mathfrak{u}}_{\mathrm{ap}}(y, m) \\
& \quad+\frac{1}{2} \sum_{m, m^{\prime}=1}^{\infty} \sum_{\sigma, \sigma^{\prime} \in\{-1,1\}} \mathfrak{h}_{\eta}\left(\sigma, \sigma^{\prime} ; m, m^{\prime}\right) \zeta^{m+m^{\prime}} \tau^{\frac{1}{4}\left(m^{2}+\left(m^{\prime}\right)^{2}\right)} \\
& \quad \times \mathfrak{v}(\lambda, \sigma, m) \mathfrak{v}\left(\lambda^{\prime}, \sigma^{\prime}, m^{\prime}\right) \mathfrak{u}_{\mathrm{u}}(\sigma, m) \mathfrak{u}_{\mathrm{u}}\left(\sigma^{\prime}, m^{\prime}\right), \\
& \widetilde{K}_{1,2}^{\eta}\left(\lambda, \lambda^{\prime}\right)=-\frac{1}{2} \sum_{m=1}^{\infty} \sum_{\sigma \in\{-1,1\}} \sigma \zeta^{m} \tau^{\frac{1}{4} m^{2}} \mathfrak{v}(\lambda, \sigma, m) \mathfrak{u}_{\mathrm{u}}(\sigma, m), \\
& \widetilde{K}_{2,2}^{\eta}\left(\lambda, \lambda^{\prime}\right)=\frac{1}{2} \operatorname{sgn}\left(\lambda^{\prime}-\lambda\right) .
\end{aligned}
$$

Up to here we have proceeded rigorously. In what follows we will proceed with a formal asymptotic analysis. The first step, which we undertake in the rest of this subsection, is to use a Mellin-Barnes representation (Lemma A.1, however, without verifying the necessary decay conditions) to argue formally that the right hand side of (A.3) yields

$$
\operatorname{Pf}\left[J-\widetilde{K}^{0}\right]
$$

with

$$
\begin{aligned}
& \widetilde{K}_{1,1}^{0}\left(\lambda, \lambda^{\prime}\right)=\frac{1}{(2 \pi \mathrm{i})^{2}} \int_{(c+\mathrm{i} \mathbb{R})^{2}} d s d s^{\prime} \frac{\pi^{2}}{\sin (-\pi s) \sin \left(-\pi s^{\prime}\right)} \mathfrak{s}_{0}\left(s, s^{\prime}\right) \\
& \times \zeta^{-s-s^{\prime}} \frac{1}{\pi \mathrm{i}} \int_{C_{0,1}} d y(-\zeta)^{2 s} \tau^{\frac{1}{2} s^{2}} \mathfrak{v}(\lambda, y, s) \mathfrak{v}\left(\lambda^{\prime}, \frac{1}{y}, s\right) \tilde{\mathfrak{u}}_{\mathrm{ap}}(y, s) \\
& +\frac{1}{2(2 \pi \mathrm{i})^{2}} \int_{(c+\mathrm{i} \mathbb{R})^{2}} d s d s^{\prime} \frac{\pi^{2}}{\sin (-\pi s) \sin \left(-\pi s^{\prime}\right)} \sum_{\sigma, \sigma^{\prime} \in\{-1,1\}} \mathfrak{h}_{0}\left(\sigma, \sigma^{\prime} ; s, s^{\prime}\right) \\
& \times(-\zeta)^{s+s^{\prime}} \tau^{\frac{1}{4}\left(s^{2}+\left(s^{\prime}\right)^{2}\right)} \mathfrak{v}(\lambda, \sigma, s) \mathfrak{v}\left(\lambda^{\prime}, \sigma^{\prime}, s^{\prime}\right) \mathfrak{u}_{\mathrm{u}}(\sigma, s) \mathfrak{u}_{\mathrm{u}}\left(\sigma^{\prime}, s^{\prime}\right), \\
& \widetilde{K}_{1,2}^{0}\left(\lambda, \lambda^{\prime}\right)=-\frac{1}{4 \pi \mathrm{i}} \int_{c+\mathrm{i} \mathbb{R}} d s \frac{\pi}{\sin (-\pi s)} \sum_{\sigma \in\{-1,1\}} \sigma(-\zeta)^{s} \tau^{\frac{1}{4} s^{2}} \mathfrak{v}(\lambda, \sigma, s) \mathfrak{u}_{\mathrm{u}}(\sigma, s), \\
& \widetilde{K}_{2,2}^{0}\left(\lambda, \lambda^{\prime}\right)=\frac{1}{2} \operatorname{sgn}\left(\lambda^{\prime}-\lambda\right),
\end{aligned}
$$

where $\mathfrak{s}_{0}\left(s, s^{\prime}\right)=\lim _{\eta \rightarrow 0} \mathfrak{s}_{\eta}\left(s, s^{\prime}\right)$ and $\mathfrak{h}_{0}\left(\sigma, \sigma^{\prime} ; s, s^{\prime}\right)=\lim _{\eta \rightarrow 0} \mathfrak{h}_{\eta}\left(\sigma, \sigma^{\prime} ; s, s^{\prime}\right)$. Note that this formula involves using first the Mellin-Barnes representation and then computing the limit $\eta \rightarrow 0$. As we will see below, it is only in the limit $\eta \rightarrow 0$ that the additional poles which arise in the Mellin-Barnes representation cancel.

In view of (A.3), and taking the $\eta \rightarrow 0$ limit back inside the Pfaffian, our goal is to argue that $\lim _{\eta \rightarrow 0} \widetilde{K}^{\eta}$ is given by $\widetilde{K}^{0}$. Consider first applying the Mellin-Barnes representation to the kernel $\widetilde{K}_{1,2}^{\eta}$. One can check that in this case the only poles of the integrand (in $s$ ) are the ones occurring at $s \in \mathbb{Z}_{\geq 1}$ coming from $\pi / \sin (-\pi s)$, and thus the Mellin-Barnes representation can be applied without additional difficulties (in other words the set $\mathbb{A}$ in Lemma A.1 is empty in this case). Doing this and taking $\eta \rightarrow 0$ yields $\widetilde{K}_{1,2}^{0}$. Note also that $\widetilde{K}_{2,2}^{\eta}$ does not depend on $\eta$, and in fact it equals $\widetilde{K}_{2,2}^{0}$, so the equality is direct in this case.

It remains to handle $\widetilde{K}_{1,1}^{\eta}$, which is where the additional poles in the Mellin-Barnes representation arise. Write for convenience

$$
\widetilde{K}_{1,1}^{\eta}=L_{1}^{\eta}+L_{2}^{\eta}
$$


where $L_{1}^{\eta}$ and $L_{2}^{\eta}$ correspond to each of the two terms appearing in the definition of $\widetilde{K}_{1,1}^{\eta}$. For $L_{1}^{\eta}$ one checks again that the only poles occur at $s, s^{\prime} \in \mathbb{Z}_{\geq 1}$ so the Mellin-Barnes representation can be applied as before, yielding

$$
\begin{aligned}
L_{1}^{\eta}=\frac{1}{(2 \pi \mathrm{i})^{2}} \int_{(c+\mathrm{i} \mathbb{R})^{2}} d s d s^{\prime} & \frac{\pi^{2}}{\sin (-\pi s) \sin \left(-\pi s^{\prime}\right)} \mathfrak{s}_{\eta}\left(s, s^{\prime}\right) \\
& \times(-\zeta)^{-s-s^{\prime}} \frac{1}{\pi \mathrm{i}} \int_{C_{0,1}} d y \zeta^{2 s} \tau^{\frac{1}{2} s^{2}} \mathfrak{v}(\lambda, y, s) \mathfrak{v}\left(\lambda^{\prime}, \frac{1}{y}, s\right) \tilde{\mathfrak{u}}_{\mathrm{ap}}(y, s)
\end{aligned}
$$

for $c \in(0,1)$. Taking $\eta \rightarrow 0$ will yield the desired representation.

Now write $L_{2}^{\eta}$ as

$$
L_{2}^{\eta}=\frac{1}{2} \sum_{m, m^{\prime}=1}^{\infty} \sum_{\sigma, \sigma^{\prime} \in\{-1,1\}} \zeta^{m+m^{\prime}} \mathfrak{h}_{\eta}\left(m, m^{\prime} ; \sigma, \sigma^{\prime}\right) I\left(m, m^{\prime} ; \sigma, \sigma^{\prime}\right)
$$

with $I\left(m, m^{\prime} ; \sigma, \sigma^{\prime}\right)=\tau^{\frac{1}{4}\left(m^{2}+\left(m^{\prime}\right)^{2}\right)} \mathfrak{v}(\lambda, \sigma, m) \mathfrak{v}\left(\lambda^{\prime}, \sigma^{\prime}, m^{\prime}\right) \mathfrak{u}_{\mathrm{u}}(\sigma, m) \mathfrak{u}_{\mathbf{u}}\left(\sigma^{\prime}, m^{\prime}\right)$. Our goal is to get

$$
\lim _{\eta \rightarrow 0} L_{2}^{\eta}=\frac{1}{2(2 \pi \mathrm{i})^{2}} \int_{(c+\mathrm{i} \mathbb{R})^{2}} d s d s^{\prime} \frac{\pi^{2}(-\zeta)^{s+s^{\prime}}}{\sin (-\pi s) \sin \left(-\pi s^{\prime}\right)} \sum_{\sigma, \sigma^{\prime} \in\{-1,1\}} \mathfrak{h}_{0}\left(s, s^{\prime} ; \sigma, \sigma^{\prime}\right) I\left(s, s^{\prime} ; \sigma, \sigma^{\prime}\right) .
$$

This case is more involved, due to the factor $\mathfrak{h}_{\eta}\left(s, s^{\prime} ; \sigma, \sigma^{\prime}\right)$. One can check that $I\left(s, s^{\prime} ; \sigma, \sigma^{\prime}\right)$ is analytic away from $s, s^{\prime} \in \mathbb{Z}_{\geq 1}$, and in fact the same holds for $\mathfrak{h}_{\eta}\left(s, s^{\prime} ; \sigma, \sigma^{\prime}\right)$ when $\sigma \neq \sigma^{\prime}$. Now consider the case $\sigma=\sigma^{\prime}$. Let us first fix $m$ and consider the effect of applying Lemma A.1 to the $m^{\prime}$ sum in (A.7). We need to analyze the poles (in $s^{\prime}$ ) of

$$
\mathfrak{h}_{\eta}\left(m, s^{\prime} ; \sigma, \sigma\right)=\sigma \frac{\Gamma\left(\frac{1}{2}\left(m-s^{\prime}\right)+\eta\right) \Gamma\left(\frac{1}{2}\left(s^{\prime}-m\right)+\eta\right)}{\Gamma\left(\frac{1}{2}\left(m+s^{\prime}\right)+\eta\right) \Gamma\left(-\frac{1}{2}\left(m+s^{\prime}\right)+\eta\right)} \frac{s^{\prime}-m+2 \eta}{m+s^{\prime}+2 \eta} .
$$

The numerator has singularities when $s^{\prime}=m+2 \ell+2 \eta$ and $s^{\prime}=m-2 \ell-2 \eta$ for $\ell \in \mathbb{Z}_{\geq 0}$. The first type of singularity is removable, because at that point the factor $1 / \Gamma\left(-\frac{1}{2}\left(m+s^{\prime}\right)+\eta\right)$ evaluates to $1 / \Gamma(-m-\ell)=0$. The second type of singularity is, on the other hand, a pole, with residue $2 \sigma \frac{(-1)^{\ell}}{\ell !} \frac{\Gamma(\ell+2 \eta)}{\Gamma(m-\ell) \Gamma(\ell-m+2 \eta)} \frac{\ell+2 \eta}{\ell-m}$, and which belongs to the deformation region (namely $\left\{z \in \mathbb{C}: \Re(z) \geq \frac{1}{2}\right\}$ ) only when $\ell<m / 2$ (assuming that $\eta$ is small). In view of this, Lemma A.1 suggests that

$$
\begin{aligned}
L_{2}^{\eta}= & \sum_{m=1}^{\infty} \frac{1}{4 \pi \mathrm{i}} \int_{c+\mathrm{i} \mathbb{R}} d s^{\prime} \frac{\pi}{\sin \left(-\pi s^{\prime}\right)} \sum_{\sigma, \sigma^{\prime} \in\{-1,1\}} \zeta^{m}(-\zeta)^{s^{\prime}} \mathfrak{h}_{\eta}\left(\sigma, \sigma^{\prime} ; m, s^{\prime}\right) I\left(\sigma, \sigma^{\prime} ; m, s^{\prime}\right) \\
& -\sum_{m=1}^{\infty} \sum_{\ell=0}^{\lfloor m / 2\rfloor} \sum_{\sigma \in\{-1,1\}} \sigma \frac{(-1)^{\ell}}{\ell !} \frac{\Gamma(\ell+2 \eta)}{\Gamma(m-\ell) \Gamma(\ell-m+2 \eta)} \frac{\ell-2 \eta}{\ell-m} \zeta^{m}(-\zeta)^{m-2 \ell-2 \eta} \\
& \times \frac{\pi}{\sin (\pi(2 \eta+2 \ell-m))} I\left(\sigma, \sigma^{\prime} ; m, m-2 \ell-2 \eta\right) \\
:=L_{2,1}^{\eta}-L_{2,2}^{\eta} . &
\end{aligned}
$$

Here we are choosing the contour $C_{1,2, \ldots}$ in Lemma A.1 to be $c+\mathrm{i} \mathbb{R}$. Now we need to apply the Mellin-Barnes representation to the remaining sum (in $m$ ) appearing in $L_{2,1}^{\eta}$, regarding $s^{\prime}$ as fixed. As before most of the factors making up the integrand are analytic, except for $\mathfrak{h}_{\eta}\left(s, s^{\prime} ; \sigma, \sigma^{\prime}\right)$ when $\sigma=\sigma^{\prime}$, in which case it reads

$$
\mathfrak{h}_{\eta}\left(s, s^{\prime} ; \sigma, \sigma\right)=\sigma \frac{\Gamma\left(\frac{1}{2}\left(s-s^{\prime}\right)+\eta\right) \Gamma\left(\frac{1}{2}\left(s^{\prime}-s\right)+\eta\right)}{\Gamma\left(\frac{1}{2}\left(s+s^{\prime}\right)+\eta\right) \Gamma\left(-\frac{1}{2}\left(s+s^{\prime}\right)+\eta\right)} \frac{s^{\prime}-s+2 \eta}{s+s^{\prime}+2 \eta} .
$$

The numerator has singularities when $s=s^{\prime}-2 \ell-2 \eta$ and $s=s^{\prime}+2 \ell+2 \eta$ for $\ell \in \mathbb{Z}_{\geq 0}$. Note that the singularities of the first type are never in the deformation region, so we are 
only left with the second type of singularities, which lie in the deformation region for all $\ell \geq 0$, with residue $2 \sigma \frac{(-1)^{\ell}}{\ell !} \frac{\Gamma(\ell+2 \eta)}{\Gamma\left(s^{\prime}+\ell+2 \eta\right) \Gamma\left(-s^{\prime}-\ell\right)} \frac{\ell}{s^{\prime}+\ell+2 \eta}$. Reasoning as before we get

$$
\begin{aligned}
L_{2,1}^{\eta}= & \sum_{\sigma, \sigma^{\prime} \in\{-1,1\}} \frac{1}{2(2 \pi \mathrm{i})^{2}} \int_{(c+\mathrm{i} \mathbb{R})^{2}} d s d s^{\prime} \frac{\pi^{2}(-\zeta)^{s+s^{\prime}}}{\sin (-\pi s) \sin \left(-\pi s^{\prime}\right)} \mathfrak{h}_{\eta}\left(s, s^{\prime} ; \sigma, \sigma^{\prime}\right) I\left(s, s^{\prime} ; \sigma, \sigma^{\prime}\right) \\
& \quad-\sum_{\ell \geq 0} \sum_{\sigma \in\{-1,1\}} \frac{1}{2 \pi \mathrm{i}} \int_{c+\mathrm{i} \mathbb{R}} d s^{\prime} \sigma \frac{(-1)^{\ell}}{\ell !} \frac{\Gamma(\ell+2 \eta)}{\Gamma\left(s^{\prime}+\ell+2 \eta\right) \Gamma\left(-s^{\prime}-\ell\right)} \frac{\ell}{s^{\prime}+\ell+2 \eta} \\
& \quad \times \frac{\pi^{2}(-\zeta)^{2 s^{\prime}+2 \ell+2 \eta}}{\sin \left(-\pi\left(s^{\prime}+2 \ell+2 \eta\right)\right) \sin \left(-\pi s^{\prime}\right)} I\left(s^{\prime}+2 \ell+2 \eta, s^{\prime} ; \sigma, \sigma\right) \\
:=L_{2,3}^{\eta}- & L_{2,4}^{\eta} .
\end{aligned}
$$

In terms of the above kernels we have $\widetilde{K}_{1,2}^{\eta}=L_{1}^{\eta}+L_{2,3}^{\eta}-L_{2,2}^{\eta}-L_{2,4}^{\eta}$. Observe now, using (A.6) and the last equation, that the limit as $\eta \rightarrow 0$ of $L_{1}^{\eta}+L_{2,3}^{\eta}$ yields exactly $\widetilde{K}_{1,1}^{0}$ (given in (A.5)), so all that remains to show is that $L_{2,2}^{\eta}+L_{2,4}^{\eta} \longrightarrow 0$ as $\eta \rightarrow 0$. For the first term, noting that the zero of the sine in the denominator cancels with a zero coming from one of the Gamma functions, so that $\frac{\pi}{\sin (\pi(2 \eta+2 \ell-m)) \Gamma(\ell-m+2 \eta)} \longrightarrow(-1)^{\ell}(m-\ell)$ !, we have:

$$
\begin{aligned}
\lim _{\eta \rightarrow 0} L_{2,2}^{\eta} & =-\sum_{m=1}^{\infty} \sum_{\ell=0}^{\lfloor m / 2\rfloor} \sum_{\sigma \in\{-1,1\}} \sigma(-1)^{m} \zeta^{2 m-2 \ell} I(\sigma, \sigma ; m, m-2 \ell) \\
& =-\sum_{\ell=0}^{\infty} \sum_{m=0}^{\infty} \sum_{\sigma \in\{-1,1\}} \sigma(-1)^{m} \zeta^{2 m+2 \ell} I(\sigma, \sigma ; m+2 \ell, m)
\end{aligned}
$$

where, for convenience, in the second equality we have added the term with $\ell=m=0$ which is 0 anyway because $\mathfrak{u}_{\mathrm{u}}(\sigma, 0)$ (which appears in $\left.I(\sigma, \sigma ; 0,0)\right)$ is so. On the other hand we have

$$
\begin{aligned}
\lim _{\eta \rightarrow 0} L_{2,4}^{\eta}=\sum_{\ell \geq 0} \sum_{\sigma \in\{-1,1\}} \frac{1}{2 \pi \mathrm{i}} \int_{c+\mathrm{i} \mathbb{R}} d s^{\prime} \sigma \frac{(-1)^{\ell}}{\ell !} \frac{\Gamma(\ell)}{\Gamma\left(s^{\prime}+\ell\right) \Gamma\left(-s^{\prime}-\ell\right)} \frac{\ell}{s^{\prime}+\ell} \\
\times \frac{\pi^{2}(-\zeta)^{2 s^{\prime}+2 \ell}}{\sin \left(-\pi\left(s^{\prime}+2 \ell\right) \sin \left(-\pi s^{\prime}\right)\right.} I\left(s^{\prime}+2 \ell, s^{\prime} ; \sigma, \sigma\right) .
\end{aligned}
$$

The $s^{\prime}$ integral can be computed in terms of the residues of the integrand for $\Re\left(s^{\prime}\right) \geq \frac{1}{2}$. There is a double zero in the denominator coming from the sine factors when $s^{\prime}=m^{\prime} \in \mathbb{Z}_{\geq 1}$, but one of them is canceled by the zero of $1 / \Gamma\left(-s^{\prime}-\ell\right)$ at these points, resulting in a simple pole, with $\operatorname{Res}_{s^{\prime}=m} \pi^{2} /\left[\sin \left(-\pi\left(s^{\prime}+2 \ell\right)\right) \sin \left(-\pi s^{\prime}\right) \Gamma\left(-s^{\prime}-\ell\right)\right]=(-1)^{m^{\prime}+\ell}\left(m^{\prime}+\ell\right)$ !. We get

$$
\lim _{\eta \rightarrow 0} L_{2,4}^{\eta}=\sum_{\ell \geq 0} \sum_{m^{\prime} \geq 1} \sum_{\sigma \in\{-1,1\}} \sigma(-1)^{m^{\prime}} \zeta^{2 m^{\prime}+2 \ell} \tau^{\frac{1}{4}\left(\left(m^{\prime}+2 \ell\right)^{2}+\left(m^{\prime}\right)^{2}\right)} I\left(\sigma, \sigma ; m^{\prime}+2 \ell, m^{\prime}\right) .
$$

As before we may add the term with $\ell=m^{\prime}=0$, and now comparing with (A.8) we see that $\lim _{\eta \rightarrow 0}\left(L_{2,2}^{\eta}+L_{2,4}^{\eta}\right)=0$ as desired, which finishes our derivation of (A.4).

A.2. Computation of the limit. We are finally in position to compute the $t \rightarrow \infty$ asymptotics of the flat ASEP distribution function. We take $\zeta=-\tau^{-\frac{1}{4} t+\frac{1}{2} t^{1 / 3} r}$ and, in view (A.4), we need to compute the limit of $\operatorname{Pf}\left[J-\widetilde{K}^{0}\right]$, with $\widetilde{K}^{0}$ given in (A.5). We will only provide a formal critical point derivation of the limit. To that end we study first the factors in $\widetilde{K}^{0}\left(s, s^{\prime}\right)$ which depend on $t$. They come from the products of the form $\tau^{\left(-\frac{1}{4} t+\frac{1}{2} t^{1 / 3} r\right) s} \mathfrak{v}(\lambda, y, s)$, and are given by

$$
\exp \left(t\left[\frac{1}{1+\tau^{-s / 2} y}-\frac{1}{1+\tau^{s / 2} y}-\frac{1}{4} \log (\tau) s\right]+\frac{1}{2} t^{1 / 3} r s \log (\tau)\right)
$$


(this factor appears twice in $\widetilde{K}_{1,1}^{0}\left(\lambda, \lambda^{\prime} ; s, s^{\prime}\right)$, once with respect to $s$ and once with respect to $s^{\prime}$, and once in $\left.\widetilde{K}_{1,2}^{0}\left(\lambda, \lambda^{\prime} ; s, s^{\prime}\right)\right)$. Consider now the function $f(s, y)=\frac{1}{1+\tau^{-s / 2} y}-\frac{1}{1+\tau^{s / 2} y}-$ $\frac{1}{4} \log (\tau) s$. One checks that $f$ has a critical point at $(0,1)$ and, moreover, that the Hessian of this function vanishes at this point. This suggests that we should use a $t^{-1 / 3}$ scaling around these points. Explicitly, we will rescale as follows:

$$
y=1+t^{-1 / 3} \widetilde{y}, \quad s=\frac{-1}{\log (\tau)} t^{-1 / 3} \widetilde{s} \quad \text { and } \quad \lambda=t^{1 / 3}(\widetilde{\lambda}-2 r)
$$

(and similarly for $\lambda^{\prime}$ and $s^{\prime}$ ). The $\lambda$ scaling is so that $\lambda \frac{1-\tau^{s / 2} y}{1+\tau^{s / 2} y}$ is order 1 (the shift by $2 r$ is for convenience). The $\lambda$ change of variables produces a $t^{1 / 3}$ in front of the kernel $\widetilde{K}^{0}$, and using (B.4) this can be rewritten as

$$
t^{1 / 3} \widetilde{K}^{0}\left(\lambda, \lambda^{\prime}\right)=\left[\begin{array}{cc}
t^{2 / 3} \widetilde{K}_{1,1}^{0}\left(\lambda, \lambda^{\prime}\right) & t^{1 / 3} \widetilde{K}_{1,2}^{0}\left(\lambda, \lambda^{\prime}\right) \\
-t^{1 / 3} \widetilde{K}_{1,2}^{0}\left(\lambda^{\prime}, \lambda\right) & \widetilde{K}_{2,2}^{0}\left(\lambda, \lambda^{\prime}\right)
\end{array}\right] .
$$

With this scaling we have

$$
\begin{gathered}
t\left[\frac{1}{1+\tau^{-s / 2} y}-\frac{1}{1+\tau^{s / 2} y}-\frac{1}{4} \log (\tau) s\right]+\frac{1}{2} r t^{1 / 3} \log (\tau) s \approx \frac{1}{192} \widetilde{s}^{3}+\frac{1}{16} \widetilde{s} \widetilde{y}^{2}, \\
\frac{1-\tau^{s / 2} y}{1+\tau^{s / 2} y} \approx \frac{1}{4}(\widetilde{s}-2 \widetilde{y}) t^{-1 / 3}, \quad \tau^{-s / 2} \frac{1+y^{2}}{y^{2}-1} d y \approx \frac{1}{\widetilde{y}} d \widetilde{y}, \quad t^{-1 / 3} \frac{\pi}{\sin (-\pi s)} d s \approx-\frac{1}{\widetilde{s}} d \widetilde{s}, \\
\sin \left(\frac{\pi}{2}\left(2 s_{1}+1\right)\right) \frac{\sin \left(\pi\left(s_{1}-s_{2}\right)\right)}{\pi\left(s_{1}-s_{2}\right)} \approx 1, \quad \frac{\Gamma\left(\frac{1}{2}\left(s_{1}-s_{2}\right)\right) \Gamma\left(\frac{1}{2}\left(s_{2}-s_{1}\right)\right)}{\Gamma\left(\frac{1}{2}\left(s_{1}+s_{2}\right)\right) \Gamma\left(-\frac{1}{2}\left(s_{1}+s_{2}\right)\right)} \frac{s_{2}-s_{1}}{s_{1}+s_{2}} \approx \widetilde{s}_{1}+\widetilde{s}_{2} \\
\widetilde{s} \widetilde{s}_{1}
\end{gathered}
$$

and, furthermore, using Lemma A.1 in [OQR14],

$$
\frac{\left(-\tau^{-s / 2} y ; \tau\right)_{\infty}}{\left(-\tau^{s / 2} y ; \tau\right)_{\infty}} \approx 1, \quad \text { and } \quad \frac{\left(\tau^{1+s} y^{2} ; \tau\right)_{\infty}}{\left(\tau y^{2} ; \tau\right)_{\infty}} \approx 1
$$

Using these asymptotics and the definition of $\mathfrak{v}$ in (4.23), we get

$$
\begin{aligned}
\mathfrak{v}(\lambda, y, s) & \approx \frac{1}{4} t^{-1 / 3}(\widetilde{s}-2 \widetilde{y}) e^{\frac{1}{192} \widetilde{s}^{3}+\frac{1}{16} \widetilde{y} \widetilde{y}^{2}-\frac{1}{4}(\widetilde{s}-2 \widetilde{y}) \widetilde{\lambda}+\frac{1}{2} \widetilde{y} r} \\
& =-t^{-1 / 3} \partial_{\lambda} e^{\frac{1}{192} \widetilde{s}^{3}+\frac{1}{16} \widetilde{y} \widetilde{y}^{2}-\frac{1}{4}(\widetilde{s}-2 \widetilde{y}) \widetilde{\lambda}+\frac{1}{2} \widetilde{y} r} .
\end{aligned}
$$

When $y$ is replaced by $1 / y$ the above asymptotics hold with $\widetilde{y}$ replaced by $-\widetilde{y}$. At the same time, if $y=1$ then the asymptotics for $\mathfrak{v}$ hold with $\widetilde{y}=0$ while if $y=-1$ the right hand side should be replaced by 0 . The above asymptotics also give, in view of the definition of $\mathfrak{u}_{\mathrm{u}}$ in (4.7) and of $\tilde{\mathfrak{u}}_{\mathrm{ap}}$ in (A.2),

$$
\mathfrak{u}_{\mathrm{u}}( \pm 1, s) \approx 1 \quad \text { and } \quad \tilde{\mathfrak{u}}_{\mathrm{ap}}(y, s) d y \approx \frac{1}{\widetilde{y}} d \widetilde{y} .
$$

Using all this in (A.5), choosing the $s$ and $s^{\prime}$ contours to be $\frac{1}{2} t^{-1 / 3}+\mathrm{i} \mathbb{R}$, and keeping (A.9) in mind we deduce that

$$
\lim _{t \rightarrow \infty} \widetilde{K}^{0}=\bar{K}
$$

with

$$
\begin{aligned}
\bar{K}_{1,1}\left(\widetilde{\lambda}, \widetilde{\lambda}^{\prime}\right) \approx \partial_{\widetilde{\lambda}} \partial_{\widetilde{\lambda}^{\prime}} \frac{1}{\pi \mathrm{i}} f_{\mathrm{i} \mathbb{R}} d \widetilde{y} \frac{1}{(2 \pi \mathrm{i})^{2}} \int_{\left(\frac{1}{2}+\mathrm{i} \mathbb{R}\right)^{2}} d \widetilde{s} d \widetilde{s}^{\prime} \frac{1}{\widetilde{s} \widetilde{s}^{\prime}} \frac{1}{\widetilde{y}} e^{\frac{1}{96} \widetilde{s}^{3}+\frac{1}{8} \widetilde{s}^{2}-\frac{1}{4} \widetilde{s}\left(\widetilde{\lambda}+\widetilde{\lambda}^{\prime}\right)+\frac{1}{2}\left(\widetilde{\lambda}-\widetilde{\lambda}^{\prime}\right) \widetilde{y}} \\
+\partial_{\widetilde{\lambda}} \partial_{\widetilde{\lambda}^{\prime}} \frac{1}{(2 \pi \mathrm{i})^{2}} \int_{\left(\frac{1}{2}+\mathrm{i} \mathbb{R}\right)^{2}} d \widetilde{s} d \widetilde{s}^{\prime} \frac{\widetilde{s}+\widetilde{s}^{\prime}}{2 \widetilde{s} \widetilde{s}^{\prime}\left(\widetilde{s}^{\prime}-\widetilde{s}\right)} e^{\frac{1}{192}\left(\widetilde{s}^{3}+\widetilde{s}^{\prime 3}\right)-\frac{1}{4}\left(\widetilde{s} \widetilde{\lambda}+\widetilde{s}^{\prime} \widetilde{\lambda}^{\prime}\right)}, \\
\bar{K}_{1,2}\left(\widetilde{\lambda}, \widetilde{\lambda}^{\prime}\right) \approx-\frac{1}{2} \partial_{\widetilde{\lambda}} \frac{1}{2 \pi \mathrm{i}} \int_{\frac{1}{2}+\mathrm{i} \mathbb{R}} d \widetilde{s} e^{\frac{1}{192} \widetilde{s}^{3}-\frac{1}{4} \widetilde{s} \widetilde{\lambda}}, \\
\bar{K}_{2,2}\left(\widetilde{\lambda}, \widetilde{\lambda}^{\prime}\right)=\frac{1}{2} \operatorname{sgn}\left(\widetilde{\lambda}^{\prime}-\widetilde{\lambda}\right) .
\end{aligned}
$$

What remains is to rewrite $\bar{K}$ in a more convenient way (this part bears some similarities with Appendix K of [LDC12]). Let us write $\bar{K}_{1,1}=\bar{K}_{1,1}^{1}+\bar{K}_{1,1}^{2}$. Note that $\bar{K}_{1,1}^{1}$ depends 
on $\widetilde{s}^{\prime}$ only through the integral $\frac{1}{2 \pi \mathrm{i}} f_{\frac{1}{2}+\mathrm{i} \mathbb{R}} d \widetilde{s}^{\prime} \frac{1}{\widetilde{s}^{\prime}}$ which, as a principal value integral, equals $\frac{1}{2}$. Then, using the definition of the Airy function,

$$
\operatorname{Ai}(z)=\frac{1}{2 \pi \mathrm{i}} \int_{c+\mathrm{i} \mathbb{R}} d u e^{\frac{1}{3} u^{3}-u z}
$$

for $c>0$, we get (removing the tildes)

$$
\begin{aligned}
\bar{K}_{1,1}^{1}\left(\lambda_{1}, \lambda_{2}\right)= & \partial_{\lambda_{1}} \partial_{\lambda_{2}} \frac{1}{\pi \mathrm{i}} f_{\mathrm{i} \mathbb{R}} d y \frac{1}{2 \pi \mathrm{i}} \int_{c+\mathrm{i} \mathbb{R}} d s_{1} \frac{1}{2 s_{1} y} e^{\frac{1}{96} s_{1}^{3}+\frac{1}{8} s_{1} y^{2}-\frac{1}{4}\left(s_{1}-2 y\right) \lambda_{1}-\frac{1}{4}\left(s_{1}+2 y\right) \lambda_{2}} \\
= & \partial_{\lambda_{1}} \partial_{\lambda_{2}} \frac{1}{2 \pi \mathrm{i}} \int_{\mathrm{i} \mathbb{R}} d y \int_{0}^{\infty} d u \frac{1}{2 \pi \mathrm{i}} \int_{c+\mathrm{i} \mathbb{R}} d s_{1} \frac{1}{y} e^{\frac{1}{96} s_{1}^{3}+\frac{1}{8} s_{1} y^{2}-\frac{1}{4}\left(s_{1}-2 y\right) \lambda_{1}-\frac{1}{4}\left(s_{1}+2 y\right) \lambda_{2}-s_{1} u} \\
& =\partial_{\lambda_{1}} \partial_{\lambda_{2}} \frac{1}{2 \pi \mathrm{i}} \int_{\mathbb{R}} d y \int_{0}^{\infty} d u \frac{1}{y} \operatorname{Ai}\left(2^{2 / 3}\left(\frac{1}{2}\left(\lambda_{1}+\lambda_{2}\right)+y^{2}\right)+u\right) e^{\mathrm{i}\left(\lambda_{2}-\lambda_{1}\right) y} \\
& =\partial_{\lambda_{2}} \frac{1}{2^{4 / 3} \pi \mathrm{i}} \int_{\mathbb{R}} d y \int_{0}^{\infty} d u \frac{1}{y} \operatorname{Ai}^{\prime}\left(2^{2 / 3}\left(\frac{1}{2}\left(\lambda_{1}+\lambda_{2}\right)+y^{2}\right)+u\right) e^{\mathrm{i}\left(\lambda_{2}-\lambda_{1}\right) y} \\
& -\partial_{\lambda_{2}} \frac{1}{2 \pi} f_{\mathbb{R}} d y \int_{0}^{\infty} d u \operatorname{Ai}\left(2^{2 / 3}\left(\frac{1}{2}\left(\lambda_{1}+\lambda_{2}\right)+y^{2}\right)+u\right) e^{\mathrm{i}\left(\lambda_{2}-\lambda_{1}\right) y} \\
= & -\partial_{\lambda_{2}} \frac{1}{2^{7 / 3} \pi \mathrm{i}} \int_{\lambda_{1}-\lambda_{2}}^{\lambda_{2}-\lambda_{1}} d \eta f_{\mathbb{R}} d y \operatorname{Ai}\left(2^{2 / 3}\left(\frac{1}{2}\left(\lambda_{1}+\lambda_{2}\right)+y^{2}\right)\right) e^{\mathrm{i} \eta y} \\
& -\partial_{\lambda_{2}} \frac{1}{2 \pi} f_{\mathbb{R}} d y \int_{0}^{\infty} d u \operatorname{Ai}\left(2^{2 / 3}\left(\frac{1}{2}\left(\lambda_{1}+\lambda_{2}\right)+y^{2}+2^{-2 / 3} u\right)\right) e^{\mathrm{i}\left(\lambda_{2}-\lambda_{1}\right) y} .
\end{aligned}
$$

As in Appendix J of [LDC12], we will use the following identity, proved in [VSI97]:

$$
\frac{1}{2^{1 / 3} \pi} \int_{-\infty}^{\infty} d y \operatorname{Ai}\left(2^{2 / 3}\left(y^{2}+\frac{1}{2}(a+b)\right)\right) e^{\mathrm{i}(a-b) y}=\operatorname{Ai}(a) \operatorname{Ai}(b)
$$

It implies that

$$
\begin{aligned}
\bar{K}_{1,1}^{1}\left(\lambda_{1}, \lambda_{2}\right)=-\partial_{\lambda_{2}} \frac{1}{4} \int_{\lambda_{1}-\lambda_{2}}^{\lambda_{2}-\lambda_{1}} d \eta \operatorname{Ai}\left(\frac{1}{2}\left(\lambda_{1}+\lambda_{2}+\eta\right)\right) \operatorname{Ai}\left(\frac{1}{2}\left(\lambda_{1}+\lambda_{2}-\eta\right)\right) \\
\quad-\partial_{\lambda_{2}} \frac{1}{2^{2 / 3}} \int_{0}^{\infty} d u \operatorname{Ai}\left(\lambda_{1}+2^{-2 / 3} u\right) \operatorname{Ai}\left(\lambda_{2}+2^{-2 / 3} u\right) \\
=-\frac{1}{2} \int_{\lambda_{1}}^{\lambda_{2}} d \eta \operatorname{Ai}^{\prime}(\eta) \operatorname{Ai}\left(\lambda_{1}+\lambda_{2}-\eta\right)-\frac{1}{2} \operatorname{Ai}\left(\lambda_{1}\right) \operatorname{Ai}\left(\lambda_{2}\right)-\partial_{\lambda_{2}} K_{\mathrm{Ai}}\left(\lambda_{1}, \lambda_{2}\right),
\end{aligned}
$$

where $K_{\mathrm{Ai}}$ was defined in (2.16).

Now we turn to $\bar{K}_{1,1}^{2}$, which is given by

$$
\begin{aligned}
\bar{K}_{1,1}^{2}\left(\lambda_{1}, \lambda_{2}\right)=\partial_{\lambda_{1}} \partial_{\lambda_{2}} \frac{1}{2(2 \pi \mathrm{i})^{2}} \int_{(c+\mathrm{i} \mathbb{R})^{2}} d s_{1} d s_{2} \frac{s_{1}+s_{2}}{s_{1} s_{2}\left(s_{2}-s_{1}\right)} e^{\frac{1}{192}\left(s_{1}^{3}+s_{2}^{3}\right)-\frac{1}{4}\left(s_{1} \lambda_{1}+s_{2} \lambda_{2}\right)} \\
=\partial_{\lambda_{1}} \partial_{\lambda_{2}} \frac{1}{2(2 \pi \mathrm{i})^{2}} \int_{2 c+\mathrm{i} \mathbb{R}} d s_{1} \int_{c+\mathrm{i} \mathbb{R}} d s_{2} \frac{1}{s_{2}\left(s_{2}-s_{1}\right)} e^{\frac{1}{192}\left(s_{1}^{3}+s_{2}^{3}\right)-\frac{1}{4}\left(s_{1} \lambda_{1}+s_{2} \lambda_{2}\right)}-\left(\lambda_{1} \longleftrightarrow \lambda_{2}\right)
\end{aligned}
$$

where we have shifted the $s_{2}$ contour to $2 c+\mathrm{i} \mathbb{R}$ for convenience and where $\left(\lambda_{1} \longleftrightarrow \lambda_{2}\right)$ denotes the same as the previous expression with $\lambda_{1}$ and $\lambda_{2}$ interchanged. The first term 
on the right hand side can be rewritten as

$$
\begin{aligned}
& -\partial_{\lambda_{1}} \partial_{\lambda_{2}} \frac{1}{2} \frac{1}{(2 \pi \mathrm{i})^{2}} \int_{2 c+\mathrm{i} \mathbb{R}} d s_{1} \int_{c+\mathrm{i} \mathbb{R}} d s_{2} \int_{0}^{\infty} d u_{1} \int_{0}^{\infty} d u_{2} e^{\frac{1}{192}\left(s_{1}^{3}+s_{2}^{3}\right)-\frac{1}{4}\left(s_{1} \lambda_{1}+s_{2} \lambda_{2}\right)-\left(s_{1}-s_{2}\right) u_{1}-s_{2} u_{2}} \\
& =-\partial_{\lambda_{1}} \partial_{\lambda_{2}} \frac{1}{2} \frac{1}{(2 \pi \mathrm{i})^{2}} \int_{2 c+\mathrm{i} \mathbb{R}} d s_{1} \int_{c+\mathrm{i} \mathbb{R}} d s_{2} \int_{0}^{\infty} d v_{1} \int_{-v_{1}}^{\infty} d v_{2} e^{\frac{1}{192}\left(s_{1}^{3}+s_{2}^{3}\right)-\frac{1}{4}\left(s_{1} \lambda_{1}+s_{2} \lambda_{2}\right)-s_{1} v_{1}-s_{2} v_{2}} \\
& =-8 \partial_{\lambda_{1}} \partial_{\lambda_{2}} \int_{0}^{\infty} d v_{1} \int_{-v_{1}}^{\infty} d v_{2} \operatorname{Ai}\left(\lambda_{1}+4 v_{1}\right) \operatorname{Ai}\left(\lambda_{2}+4 v_{2}\right) \\
& =\frac{1}{2} \int_{0}^{\infty} d v \operatorname{Ai}^{\prime}\left(\lambda_{1}+v\right) \operatorname{Ai}\left(\lambda_{2}-v\right)=\frac{1}{2} \int_{\lambda_{1}}^{\infty} d v \operatorname{Ai}^{\prime}(v) \operatorname{Ai}\left(\lambda_{1}+\lambda_{2}-v\right),
\end{aligned}
$$

where we have used again (A.11). Subtracting the term with $\lambda_{1}$ and $\lambda_{2}$ flipped we get

$$
\bar{K}_{1,1}^{2}\left(\lambda_{1}, \lambda_{2}\right)=\frac{1}{2} \int_{\lambda_{1}}^{\lambda_{2}} d \omega \operatorname{Ai}^{\prime}(\omega) \operatorname{Ai}\left(\lambda_{1}+\lambda_{2}-\omega\right) .
$$

Note that this term cancels the first term on the right hand side of (A.12), and thus we obtain

$$
\bar{K}_{1,1}\left(\lambda_{1}, \lambda_{2}\right)=-\partial_{\lambda_{2}} K_{\mathrm{Ai}}\left(\lambda_{1}, \lambda_{2}\right)-\frac{1}{2} \operatorname{Ai}\left(\lambda_{1}\right) \operatorname{Ai}\left(\lambda_{2}\right)=\frac{1}{2}\left(\partial_{\lambda_{1}}-\partial_{\lambda_{2}}\right) K_{\mathrm{Ai}}\left(\lambda_{1}, \lambda_{2}\right),
$$

where the second equality follows by integration by parts.

$\bar{K}_{1,2}$ is simpler to obtain: using (A.11) one more time,

$$
\bar{K}_{1,2}\left(\lambda_{1}, \lambda_{2}\right)=-\frac{1}{16 \pi \mathrm{i}} \int_{c+\mathrm{i} \mathbb{R}} d s e^{\frac{1}{192} s^{3}-\frac{1}{4} s \lambda_{1}}=-\frac{1}{2} \operatorname{Ai}\left(\lambda_{1}\right) .
$$

This, together with (A.13), gives

$$
\bar{K}\left(\lambda_{1}, \lambda_{2}\right)=\left[\begin{array}{cc}
\frac{1}{2}\left(\partial_{\lambda_{1}}-\partial_{\lambda_{2}}\right) K_{\mathrm{Ai}}\left(\lambda_{1}, \lambda_{2}\right) & -\frac{1}{2} \operatorname{Ai}\left(\lambda_{1}\right) \\
\frac{1}{2} \operatorname{Ai}\left(\lambda_{2}\right) & \frac{1}{2} \operatorname{sgn}\left(\lambda_{2}-\lambda_{1}\right)
\end{array}\right] .
$$

In view of (A.4), (A.10) and the definition of $K_{r}$ in (2.15) (shifting $\lambda_{1} \mapsto \lambda_{1}+r, \lambda_{2} \mapsto \lambda_{2}+r$ in the Pfaffian as well), this finishes our formal asymptotic analysis of $\operatorname{Pf}\left[J-\widetilde{K}^{\text {flat, } \zeta}\right]$, yielding $\operatorname{Pf}\left[J-K_{r}\right]$.

A.3. Fredholm Pfaffian formula for GOE. All that remains is to complete our formal derivation of the GOE asymptotics for flat ASEP is to verify that the limiting Fredholm Pfaffian is indeed a formula for the Tracy-Widom GOE distribution. This fact can be proved rigorously:

\section{Proposition A.4.}

with

$$
F_{\mathrm{GOE}}(r)=\operatorname{Pf}\left[J-K_{r}\right]_{L^{2}([0, \infty))},
$$

$$
K_{r}\left(\lambda_{1}, \lambda_{2}\right)=\left[\begin{array}{cc}
\frac{1}{2}\left(\partial_{\lambda_{1}}-\partial_{\lambda_{2}}\right) K_{\mathrm{Ai}}\left(\lambda_{1}+r, \lambda_{2}+r\right) & -\frac{1}{2} \operatorname{Ai}\left(\lambda_{1}+r\right) \\
\frac{1}{2} \operatorname{Ai}\left(\lambda_{2}+r\right) & \frac{1}{2} \operatorname{sgn}\left(\lambda_{2}-\lambda_{1}\right)
\end{array}\right]
$$

A very similar formula for $F_{\mathrm{GOE}}$ appears in [Fer04]. Verifying that the Fredholm Pfaffian on the right hand side of (A.14) is convergent is not hard. In fact, it is enough to note that the kernel $K_{r}$ is uniformly bounded for $\lambda_{1}, \lambda_{2} \geq 0$, and then expand the Pfaffian as a Fredholm series and use the identity $\operatorname{Pf}[A]^{2}=\operatorname{det}(\bar{A})$ together with Hadamard's bound.

In view of the discussion about Fredholm Pfaffians contained in Appendix B.3 (see in particular (B.17)), it would be nice if one could find a symplectic $2 \times 2$-matrix kernel $M$ so that $M^{\top} K_{r} M$ is trace class. Unfortunately, it does not seem like such a kernel exists. More precisely, at least in the Fredholm determinant case one is usually led to consider 
multiplication operators for conjugation (see (B.10)). In the symplectic case such an operator would be of the form $M\left(\begin{array}{c}f_{1} \\ f_{2}\end{array}\right)(x)=\left(\begin{array}{c}\left.\phi(x) f_{1}(x)\right) \\ \phi(x)^{-1} f_{2}(x)\end{array}\right)$ for some (non-vanishing) function $\phi$. But it is proved in page 82 of [DG09] that there is no choice of $\phi$ for which $M^{\top} K_{r} M$ is trace class (the underlying problem is the singularity at the diagonal of $\operatorname{sgn}\left(\lambda_{2}-\lambda_{1}\right)$ ). As mentioned at the end of Appendix B.3, we can still use the relation between Fredholm Pfaffians and Fredholm determinants since we know that the Fredholm Pfaffian in (A.14) and the associated Fredholm determinant (see below) define absolutely convergent series. This is what we will do in the proof, which will then be devoted to showing that the resulting Fredholm determinant yields $F_{\mathrm{GOE}}$. The proof follows the arguments of Section 7 of [LDC12] relatively closely.

Proof of Proposition A.4. For convenience will omit the subscript $r$ in $K_{r}$ during this proof. We will also omit the subscripts from Fredholm determinants and Pfaffians, which are always computed on $L^{2}([0, \infty))$ or $L^{2}([0, \infty)) \otimes L^{2}([0, \infty))$.

We have shown already that the Fredholm Pfaffian series for $\operatorname{Pf}[J-K]$ is absolutely convergent. An identical argument shows that $\operatorname{det}[I+J K]$ satisfies the same, and thus Proposition B.4 implies that

$$
\operatorname{Pf}[J-K]^{2}=\operatorname{det}[I+J K]=\operatorname{det}\left[\begin{array}{cc}
I-K_{1,2}^{\top} & K_{2,2} \\
-K_{1,1} & I-K_{1,2}
\end{array}\right] .
$$

In view of (A.13), let us write

$$
K_{1,1}=K_{1,1}^{a}+K_{1,1}^{b}
$$

with

$$
K_{1,1}^{a}\left(\lambda_{1}, \lambda_{2}\right)=-\partial_{\lambda_{2}} K_{\mathrm{Ai}}\left(\lambda_{1}+r, \lambda_{2}+r\right) \quad \text { and } \quad K_{1,1}^{2}\left(\lambda_{1}, \lambda_{2}\right)=-\frac{1}{2} \operatorname{Ai}\left(\lambda_{1}+r\right) \operatorname{Ai}\left(\lambda_{2}+r\right)
$$

Note that $K_{1,1}^{b}$ is a symmetric, rank-one kernel. We claim that $K_{1,1}$ can be replaced by $K_{1,1}^{a}$ on the right hand side of (A.15):

$$
\operatorname{Pf}[J-K]^{2}=\operatorname{det}\left[\begin{array}{cc}
I-K_{1,2}^{\top} & K_{2,2} \\
-K_{1,1}^{a} & I-K_{1,2}
\end{array}\right] .
$$

It is enough to check this at the level of the finite dimensional determinants which appear in Fredholm determinant series (B.7) for $\operatorname{det}[I+J K]$, in which case it follows from the following general fact: if $A, B, C, U$ are $n \times n$ (real) matrices with $B$ and $C$ skew-symmetric and $U$ symmetric and rank-one, then the matrices

$$
\left[\begin{array}{cc}
A & B \\
C+U & A^{\top}
\end{array}\right] \quad \text { and } \quad\left[\begin{array}{cc}
A & B \\
C & A^{\top}
\end{array}\right]
$$

have the same eigenvalues (and eigenvectors) To see this, suppose that $\left(v_{1}, v_{2}\right)$ is an eigenvector of $\left[\begin{array}{cc}A & B \\ C+U & A^{\top}\end{array}\right]$, where $v_{i} \in \mathbb{R}^{n}$. We claim that $U v_{1}=0$, which implies that $\left(v_{1}, v_{2}\right)$ is also an eigenvector of $\left[\begin{array}{cc}A & B \\ C & A^{\top}\end{array}\right]$ with the same eigenvalue, say $\lambda$. In fact, we have $A v_{1}+B v_{2}=\lambda v_{1}$ and $(C+U) v_{1}+A^{\top} v_{2}=\lambda v_{2}$. Now test the first equation on the right with $v_{2}$ and the second on the left with $v_{1}$ to get $\left\langle A v_{1}, v_{2}\right\rangle+\left\langle B v_{2}, v_{2}\right\rangle=\lambda\left\langle v_{1}, v_{2}\right\rangle$ and $\left\langle v_{1},(C+U) v_{1}\right\rangle+\left\langle v_{1}, A^{\top} T v_{2}\right\rangle=$ $\lambda\left\langle v_{1}, v_{2}\right\rangle$. By skew-symmetry we have $\left\langle B v_{2}, v_{2}\right\rangle=\left\langle v_{1}, C v_{1}\right\rangle=0$, so we conclude from the two equations that $\left\langle v_{1}, U v_{1}\right\rangle=0$. Now a symmetric rank one matrix must be a multiple of a projection onto a vector $w$ and we conclude that $\left\langle v_{1}, w\right\rangle=0$ which further implies that $U v_{1}=0$. This proves our claim that the matrices in (A.17) have the same eigenvalues, and thus (A.16).

Since $K_{1,2}$ is rank-one, the kernel inside the determinant on the right hand side of (A.16) is a rank-two perturbation of $\left[\begin{array}{cc}I & K_{2,2} \\ -K_{1,1}^{a} & I\end{array}\right]$. Now recall that if $B$ is a rank-one kernel then

$$
\operatorname{det}[I+A+B]=\operatorname{det}[I+A]\left(1+\operatorname{tr}\left[(I+A)^{-1} B\right]\right)
$$


(this will be used later on), as long as $I+A$ is invertible. A similar formula holds for rank-two perturbations, but in our case the skew-symmetry of $K_{1,1}^{a}$ and $K_{2,2}$ yields the nicer formula

$$
\begin{aligned}
\operatorname{Pf}[J-K]^{2}=\operatorname{det}\left[I+\left[\begin{array}{cc}
0 & K_{2,2} \\
-K_{1,1}^{a} & 0
\end{array}\right]-\left[\begin{array}{cc}
K_{1,2}^{\top} & 0 \\
0 & K_{1,2}
\end{array}\right]\right] \\
\quad=\operatorname{det}\left[I+K_{1,1}^{a} K_{2,2}\right]\left(1-\operatorname{tr}\left[\left(I+K_{1,1}^{a} K_{2,2}\right)^{-1} K_{1,2}\right]\right)^{2} .
\end{aligned}
$$

Of course, a necessary condition for this identity to hold is that $I+K_{1,1}^{a} K_{2,2}$ be invertible, but this is true thanks to (A.22). The identity follows by approximation from the following matrix identity, which is (167) in [LDC12]: if $A$ and $B$ are skew-symmetric $n \times n$ matrices, $R=u v^{\top}$ for some $u, v \in \mathbb{R}^{n}, Q=\left[\begin{array}{cc}R & 0 \\ 0 & R^{T}\end{array}\right]$ and $P=\left[\begin{array}{cc}I & B \\ A & I\end{array}\right]$, then

$$
\operatorname{det}(P+Q)=\operatorname{det}(P)\left(1+\left\langle u,(I-A B)^{-1} v\right\rangle\right)^{2},
$$

as long as $I-A B$ is invertible. Since this matrix identity is not completely obvious, and no proof is given in [LDC12], let us pause for a moment to prove it.

Note first that, since $I-A B$ is invertible by assumption (and then, taking transpose and by skew-symmetry of $A$ and $B$, so is $I-B A)$, we have $P^{-1}=\left[\begin{array}{cc}(I-B A)^{-1} & 0 \\ 0 & (I-A B)^{-1}\end{array}\right]\left[\begin{array}{cc}I & -B \\ -A & I\end{array}\right]$. On the other hand, we have $\operatorname{det}(P+Q)=\operatorname{det}(P) \operatorname{det}\left(I+P^{-1} Q\right)$, so it suffices to show that $\operatorname{det}\left(I+P^{-1} Q\right)=\left(1+\left\langle u,(I-A B)^{-1} v\right\rangle\right)^{2}$. Using the formula for $P^{-1}$ we get $\left(P^{-1} Q\right)\left(\begin{array}{c}w_{1} \\ w_{2}\end{array}\right)=$ $\left(\begin{array}{c}\left\langle v, w_{1}\right\rangle(I-B A)^{-1} u \\ \left\langle u, w_{2}\right\rangle(I-A B)^{-1} v\end{array}\right)-\left(\begin{array}{c}\left\langle u, w_{2}\right\rangle(I-B A)^{-1} B v \\ \left\langle v, w_{1}\right\rangle(I-A B)^{-1} A u\end{array}\right)$. Now since $A$ and $B$ are skew-symmetric, we have (taking transpose) that $\left\langle u,(I-A B)^{-1} A u\right\rangle=-\left\langle u, A(I-B A)^{-1} u\right\rangle$. But $(I-A B)^{-1} A=$ $A(I-B A)^{-1}$, as can be checked easily by multiplying both sides by $I-A B$, so the previous identity implies that $\left\langle u,(I-A B)^{-1} A u\right\rangle=0$. Now it is easy to see that $P^{-1} Q$ is rank-two, with eigenfunctions $\left(\begin{array}{c}(I-B A)^{-1} u \\ (I-A B)^{-1} A u\end{array}\right)$ and $\left(\begin{array}{c}(I-B A)^{-1} B v \\ (I-A B)^{-1} v\end{array}\right)$, both with eigenvalue $\left\langle u,(I-A B)^{-1} v\right\rangle$. This implies that $\operatorname{det}\left(I+P^{-1} Q\right)=\left(1+\left\langle u,(I-A B)^{-1} v\right\rangle\right)^{2}$ as desired, and (A.20) follows.

Going back to (A.19), observe that

$$
\begin{aligned}
K_{1,1}^{a} K_{2,2}\left(\lambda_{1}, \lambda_{2}\right) & =-\frac{1}{2} \int_{0}^{\infty} d \xi \partial_{\xi} K_{\mathrm{Ai}}\left(\lambda_{1}+r, \xi+r\right) \operatorname{sgn}\left(\lambda_{2}-\xi\right) \\
& =-K_{\mathrm{Ai}}\left(\lambda_{1}+r, \lambda_{2}+r\right)+\frac{1}{2} K_{\mathrm{Ai}}\left(\lambda_{1}+r, r\right)
\end{aligned}
$$

Writing

$$
B_{r}\left(\lambda_{1}, \lambda_{2}\right)=\operatorname{Ai}\left(\lambda_{1}+\lambda_{2}+r\right) \quad \text { and } \quad \bar{\delta}_{0}=\delta_{0} \otimes \mathbf{1}
$$

(here 1 is the function which is identically equal to 1 ), observing that $K_{\mathrm{Ai}}(\cdot+r, \cdot+r)=B_{r}^{2}$ and using the last formula we may write

$$
K_{1,1}^{a} K_{2,2}=-B_{r}^{2}+\frac{1}{2} B_{r}^{2} \bar{\delta}_{0} \quad \text { and } \quad K_{1,2}=-\frac{1}{2} B_{r} \bar{\delta}_{0} .
$$

The kernel $\frac{1}{2} B_{r}^{2} \bar{\delta}_{0}$ is rank-one, and thus by (A.18) we have

$$
\operatorname{det}\left[I+K_{1,1}^{a} K_{2,2}\right]=\operatorname{det}\left[I-B_{r}^{2}\right]\left(1+\frac{1}{2} \operatorname{tr}\left[\left(I-B_{r}^{2}\right)^{-1} B_{r}^{2} \bar{\delta}_{0}\right]\right) .
$$

On the other hand, using again the fact that $K_{1,1}^{a} K_{2,2}$ is a rank-one perturbation of $-B_{r}^{2}$, the Sherman-Morrison formula gives

$$
\left(I+K_{1,1}^{a} K_{2,2}\right)^{-1}=\left(I-B_{r}^{2}\right)^{-1}-\frac{1}{2} \frac{\left(I-B_{r}^{2}\right)^{-1} B_{r}^{2} \bar{\delta}_{0}\left(I-B_{r}^{2}\right)^{-1}}{1+\frac{1}{2} \operatorname{tr}\left[\left(I-B_{r}^{2}\right)^{-1} B_{r}^{2} \bar{\delta}_{0}\right]}
$$

and thus

$$
1+\operatorname{tr}\left[\left(I+K_{1,1}^{a} K_{2,2}\right)^{-1} K_{1,2}\right]=1-\frac{1}{2} \operatorname{tr}\left[\left(I-B_{r}^{2}\right)^{-1} B_{r} \bar{\delta}_{0}\right]+\frac{1}{4} \frac{\operatorname{tr}\left[\left(I-B_{r}^{2}\right)^{-1} B_{r}^{2} \bar{\delta}_{0}\left(I-B_{r}^{2}\right)^{-1} B_{r} \bar{\delta}_{0}\right]}{1+\frac{1}{2} \operatorname{tr}\left[\left(I-B_{r}^{2}\right)^{-1} B_{r}^{2} \bar{\delta}_{0}\right]} .
$$

Now writing $B_{r} \bar{\delta}_{0}$ as $\psi \otimes \mathbf{1}$ (where $\psi(\lambda)=\operatorname{Ai}(\lambda+r)$ ), $A=\left(I-B_{r}^{2}\right)^{-1}$ and $B=B_{r} \bar{\delta}_{0}$, one checks that

$$
\operatorname{tr}[A B(\psi \otimes \mathbf{1}) A(\psi \otimes \mathbf{1})]=\operatorname{tr}[A B(\psi \otimes \mathbf{1})] \operatorname{tr}[A(\psi \otimes \mathbf{1})]
$$


Using this fact, the above identity yields

$1+\operatorname{tr}\left[\left(I+K_{1,1}^{a} K_{2,2}\right)^{-1} K_{1,2}\right]=\frac{1-\frac{1}{2} \operatorname{tr}\left[\left(I-B_{r}^{2}\right)^{-1} B_{r} \bar{\delta}_{0}\right]+\frac{1}{2} \operatorname{tr}\left[\left(I-B_{r}^{2}\right)^{-1} B_{r}^{2} \bar{\delta}_{0}\right]}{1+\frac{1}{2} \operatorname{tr}\left[\left(I-B_{r}^{2}\right)^{-1} B_{r}^{2} \bar{\delta}_{0}\right]}=\frac{1-\frac{1}{2} \operatorname{tr}\left[\left(I+B_{r}\right)^{-1} B_{r} \bar{\delta}_{0}\right]}{1+\frac{1}{2} \operatorname{tr}\left[\left(I-B_{r}^{2}\right)^{-1} B_{r}^{2} \bar{\delta}_{0}\right]}$

Using this and (A.21) in (A.19) we deduce that

$$
\operatorname{Pf}[J-K]=\operatorname{det}\left[1-B_{r}^{2}\right] \frac{\left(1-\frac{1}{2} \operatorname{tr}\left[\left(I+B_{r}\right)^{-1} B_{r} \bar{\delta}_{0}\right]\right)^{2}}{1+\frac{1}{2} \operatorname{tr}\left[\left(I-B_{r}^{2}\right)^{-1} B_{r}^{2} \bar{\delta}_{0}\right]} .
$$

Now let $\varphi_{\varepsilon}(x)=\left(\frac{2}{\pi \varepsilon}\right)^{1 / 2} e^{-x^{2} /(2 \varepsilon)}$ for $\varepsilon>0$, so that

$$
\operatorname{tr}\left[\left(I+B_{r}\right)^{-1} B_{r} \bar{\delta}_{0}\right]=\lim _{\varepsilon \rightarrow 0} \operatorname{tr}\left[\left(I+B_{r}\right)^{-1} B_{r}\left(\varphi_{\varepsilon} \otimes \mathbf{1}\right)\right] .
$$

Since $\operatorname{tr}\left[\varphi_{\varepsilon} \otimes \mathbf{1}\right]=1$ we have

$$
1-\frac{1}{2} \operatorname{tr}\left[\left(I+B_{r}\right)^{-1} B_{r} \bar{\delta}_{0}\right]=\frac{1}{2}+\frac{1}{2} \lim _{\varepsilon \rightarrow 0} \operatorname{tr}\left[\left(I+B_{r}\right)^{-1}\left(\varphi_{\varepsilon} \otimes \mathbf{1}\right)\right]=\frac{1}{2}+\frac{1}{2} \operatorname{tr}\left[\left(I+B_{r}\right)^{-1} \bar{\delta}_{0}\right] .
$$

In a similar way we get

$$
1+\frac{1}{2} \operatorname{tr}\left[\left(I-B_{r}^{2}\right)^{-1} B_{r}^{2} \bar{\delta}_{0}\right]=\frac{1}{2}+\frac{1}{2} \operatorname{tr}\left[\left(I-B_{r}^{2}\right)^{-1} \bar{\delta}_{0}\right]=\frac{1}{2}+\frac{1}{4} \operatorname{tr}\left[\left(I-B_{r}\right)^{-1} \bar{\delta}_{0}\right]+\frac{1}{4} \operatorname{tr}\left[\left(I+B_{r}\right)^{-1} \bar{\delta}_{0}\right] .
$$

We deduce from the above identities that

$$
\operatorname{Pf}[J-K]=\operatorname{det}\left[1-B_{r}^{2}\right] \frac{\left(1+\operatorname{tr}\left[\left(I+B_{r}\right)^{-1} \bar{\delta}_{0}\right]\right)^{2}}{2+\operatorname{tr}\left[\left(I-B_{r}\right)^{-1} \bar{\delta}_{0}\right]+\operatorname{tr}\left[\left(I+B_{r}\right)^{-1} \bar{\delta}_{0}\right]} .
$$

Now we use the identity

$$
\frac{\operatorname{det}\left[I-B_{r}\right]}{\operatorname{det}\left[I+B_{r}\right]}=\operatorname{tr}\left[\left(I+B_{r}\right)^{-1} \bar{\delta}_{0}\right]
$$

proved in [FS05], and the fact, noted in [LDC12], that the same identity holds (with identical proof) if $B_{r}$ is replaced by $-B_{r}$. The consequence is that

$$
\operatorname{Pf}[J-K]^{2}=\operatorname{det}\left[1-B_{r}^{2}\right] \frac{\left(1+\frac{\operatorname{det}\left[I-B_{r}\right]}{\operatorname{det}\left[I+B_{r}\right]}\right)^{2}}{2+\frac{\operatorname{det}\left[I-B_{r}\right]}{\operatorname{det}\left[I+B_{r}\right]}+\frac{\operatorname{det}\left[I+B_{r}\right]}{\operatorname{det}\left[I-B_{r}\right]}}=\operatorname{det}\left[I-B_{r}\right]^{2} .
$$

Since, by $[\mathrm{FS} 05], \operatorname{det}\left[I-B_{r}\right]_{L^{2}([0, \infty))}=F_{\mathrm{GOE}}(r)$, this proves (A.14) up to sign.

In order to determine the sign the basic idea is to argue by continuity and compare the two sides in the limit $r \rightarrow \infty$ (in the remainder of the proof we will reintroduce the subscript in $K_{r}$ ). In order use continuity we will take advantage of the fact that, although $K_{r}$ is not trace class, it is easy to turn its Fredholm Pfaffian into that of a Hilbert-Schmidt operator (for the definition see [QR14]). In fact, defining a multiplication operator $M\left(\begin{array}{c}f_{1} \\ f_{2}\end{array}\right)(x)=\left(\begin{array}{c}\left.\phi(x) f_{1}(x)\right) \\ \phi(x)^{-1} f_{2}(x)\end{array}\right)$ with $\phi(x)=\left(1+x^{2}\right)$, it is not hard to check, using the fact that $|\operatorname{Ai}(x)| \leq c e^{-2 x^{3 / 2} / 3}$ for $x \geq 0$, that $M^{\top} K_{r} M$ is Hilbert-Schmidt (see Example 2 in Section 2 of [QR14] for the proof of a similar fact). Thus we may use the notion of regularized Pfaffians introduced in Section B.3 (see (B.18)). The idea is to study

$$
\begin{aligned}
\operatorname{Pf}_{2}\left[J-M^{\top} K_{r} M\right] & \left.=\operatorname{Pf}\left[e^{-\frac{1}{2} J M^{\top} K_{r} M}\left(J+J M^{\top} K_{r} M J\right) e^{-\frac{1}{2} M^{\top} K_{r} M J}\right]\right] \\
& =e^{\operatorname{tr}\left[\phi^{-1}\left(K_{r}\right)_{1,2} \phi\right]} \operatorname{Pf}\left[J+J M^{\top} K_{r} M J\right] .
\end{aligned}
$$

The first equality is by definition of the regularized determinant. For the second one, where $\phi$ and $\phi^{-1}$ denote the corresponding multiplication operators, we are using the fact that the operator appearing in the exponent is trace class (see Example 3 in Section 2 of [QR14] for the proof of a similar fact) to show that $\operatorname{det}\left[e^{-\frac{1}{2} M^{\top} K_{r} M J}\right]$ equals the exponential; the identity follows now from (B.16) (the fact that $\operatorname{Pf}\left[J+J M^{\top} K_{r} M J\right]$ defines a convergent series follows in the same way as for $\left.\operatorname{Pf}\left[J-K_{r}\right]\right)$.

A similar argument to the one that shows that $M^{\top} K_{r} M$ is Hilbert-Schmidt shows that $M^{\top} K_{r} M$ is continuous in $r$ in Hilbert-Schmidt norm, which implies by (B.19) that the left hand side of (A.24) is continuous in $r$. Moreover, similar arguments show that $\phi^{-1}\left(K_{r}\right)_{1,2} \phi$ is continuous in $r$ in trace class norm, and thus the exponential in (A.24) is continuous 
in $r$. We deduce that $\operatorname{Pf}\left[J+J M^{\top} K_{r} M J\right]=\operatorname{Pf}\left[J-K_{r}\right]$ is continuous in $r$ and thus, since $F_{\mathrm{GOE}}(r)>0$ for all $r$, this and (A.23) imply that there is a $\sigma \in\{-1,1\}$ such that $\operatorname{Pf}\left[J-K_{r}\right]=\sigma F_{\mathrm{GOE}}(r)$ for all $r$. Using again (B.19) and the fact that $\lim _{r \rightarrow \infty} M^{\top} K_{r} M=$ $M^{\top} K_{\infty} M$ in Hilbert-Schmidt norm with $K_{\infty}\left(\lambda_{1}, \lambda_{2}\right)=\left[\begin{array}{cc}0 & 0 \\ 0 & \frac{1}{2} \operatorname{sgn}\left(\lambda_{2}-\lambda_{1}\right)\end{array}\right]$, we get $\lim _{r \rightarrow \infty} \operatorname{Pf}[J-$ $\left.M^{\top} K_{r} M\right]=\operatorname{Pf}\left[J-M^{\top} K_{\infty} M\right]=1$. Since $\lim _{r \rightarrow \infty} F_{\mathrm{GOE}}(r)$ is also 1 , this implies that $\sigma=1$ and finishes the proof of (A.24).

\section{Appendix B. Fredholm Pfaffians}

The purpose of this section is to provide a brief discussion about the theory of Fredholm Pfaffians. These were introduced by Rains in [Rai00], who stated its main properties. Some of the issues discussed in Section B.3 are not directly motivated by what is used in the main text, but we chose to include them in the hope that they will help clarifying the notion of a Fredholm Pfaffian and its relation to the Fredholm determinant.

B.1. Pfaffians. Let $A$ be a $2 n \times 2 n$ skew-symmetric matrix. By basic spectral theory the eigenvalues of $A$ come in pairs $\pm \mathrm{i} \lambda$ (for real $\lambda$ ), which then $\operatorname{implies}$ that $\operatorname{det}(A)$ can be written as the square of a polynomial in the entries of $A$. The Pfaffian of $A$ is defined (up to sign) to be this polynomial, and it has the following explicit form:

$$
\operatorname{Pf}(A)=\frac{1}{2^{n} n !} \sum_{\sigma \in S_{2 n}} \operatorname{sgn}(\sigma) \prod_{j=1}^{n} A_{\sigma(2 j-1), \sigma(2 j)} .
$$

When $A$ has odd size the same arguments imply that $A$ has 0 as an eigenvalue, which leads to define $\operatorname{Pf}(A)=0$. The fact that the definition of the Pfaffian through (B.1) satisfies

$$
\operatorname{Pf}(A)^{2}=\operatorname{det}(A)
$$

is not at all obvious; we refer the reader to Section 3 of [DG09] which contains three different proofs.

Another basic fact about Pfaffians (a proof of which can also be found in [DG09]) is the following: Given two matrices $A, B$ of size $k \times k$, with $k$ even and $A$ skew-symmetric,

$$
\operatorname{Pf}\left(B A B^{\top}\right)=\operatorname{det}(B) \operatorname{Pf}(A) \text {. }
$$

A particular case of this identity, which we use repeatedly in Section 4 and below, is the following. Let $A, B, U$ be given matrices of size $k \times k$, with $A$ and $B$ skew-symmetric, and $D_{1}, D_{2}$ be diagonal matrices of the same size. Then

$$
\prod_{a=1}^{k}\left(D_{1}\right)_{a, a}\left(D_{2}\right)_{a, a} \operatorname{Pf}\left[\left(\begin{array}{cc}
A & U \\
-U & B
\end{array}\right)\right]=\operatorname{Pf}\left[\left(\begin{array}{cc}
D_{1} & 0 \\
0 & D_{2}
\end{array}\right)\left(\begin{array}{cc}
A & U \\
-U & B
\end{array}\right)\left(\begin{array}{cc}
D_{1} & 0 \\
0 & D_{2}
\end{array}\right)\right] .
$$

We collect here some further facts about Pfaffians of skew-symmetric matrices which were used in the main text. The first one was used to extend certain formulas from an even to an odd number of variables in Section 4.2, while the next two involve certain integration identities which were useful in the derivation of the Pfaffian formulas in Sections 4 and 5.

Lemma B.1. Fix $k \in \mathbb{Z}_{>0}$ and consider two skew-symmetric $k \times k$ matrices $A$ and $B$, two (column) vectors $U, V$ of size $k$, and define the $k \times k$ matrix $D=U V^{\top}$. Then

$$
\operatorname{Pf}\left[\begin{array}{cc}
A & D \\
-D^{\mathrm{T}} & B
\end{array}\right]=\left\{\begin{array}{cc}
\operatorname{Pf}[A] \operatorname{Pf}[B] & \text { if } k \text { is even } \\
\operatorname{Pf}\left[\begin{array}{cc}
A & U \\
-U^{\top} & 0
\end{array}\right] \operatorname{Pf}\left[\begin{array}{cc}
B & V \\
-V^{\top} & 0
\end{array}\right] & \text { if } k \text { is odd. }
\end{array}\right.
$$


Proof. Let us first consider the case $k$ even. More generally, we will consider the case where $A$ is $k_{1} \times k_{1}, B$ is $k_{2} \times k_{2}$ and $D$ is $k_{1} \times k_{2}$, with $k_{1}$ and $k_{2}$ even. If $A$ or $B$ are singular then clearly both sides vanish, so we will assume that $A$ and $B$ are invertible. We have

$$
\operatorname{Pf}\left[\begin{array}{cc}
A & D \\
-D^{\mathrm{T}} & B
\end{array}\right]^{2}=\operatorname{det}\left[\begin{array}{cc}
A & D \\
-D^{\mathrm{T}} & B
\end{array}\right]=\operatorname{det}[A] \operatorname{det}\left[B+D^{\top} A^{-1} D\right] .
$$

Now $D^{\top} A^{-1} D=V\left(U^{\top} A^{-1} U\right) V^{\top}$, and the middle factor is a scalar which, by the skewsymmetry of $A^{-1}$, has to be zero. This shows that

$$
\operatorname{Pf}\left[\begin{array}{cc}
A & D \\
-D^{\mathrm{T}} & B
\end{array}\right]^{2}=\operatorname{Pf}[A]^{2} \operatorname{Pf}[B]^{2},
$$

which implies the desired identity up to sign. To determine the sign, replace $D$ by $\varepsilon D$ for $\varepsilon \geq 0$. Since $\operatorname{Pf}\left[\begin{array}{cc}A & \varepsilon D \\ -\varepsilon D^{\mathrm{T}} & B\end{array}\right]$ is a continuous function of $\varepsilon$ which does not vanish for $\varepsilon \geq 0$ (recall that we are assuming that $A$ are $B$ are not singular) we see by (B.6) that its value is either always $\operatorname{Pf}[A] \operatorname{Pf}[B]$ or always $-\operatorname{Pf}[A] \operatorname{Pf}[B]$. Now taking $\varepsilon=0$ the Pfaffian becomes $\operatorname{Pf}\left[\begin{array}{ll}A & 0 \\ 0 & B\end{array}\right]=\operatorname{Pf}[A] \operatorname{Pf}[B]$, which shows that (B.6) holds without the squares as desired.

Now for the case $k$ odd, consider the matrix

$$
E=\left[\begin{array}{cccc}
A & D & U & U \\
-D^{\top} & B & -V & -V \\
-U^{\top} & V^{\top} & 0 & 1 \\
-U^{\top} & V^{\top} & -1 & 0
\end{array}\right] .
$$

By the previous case, $\operatorname{Pf}[E]=\operatorname{Pf}\left[\begin{array}{cc}A & D \\ -D^{\mathrm{T}} & B\end{array}\right] \operatorname{Pf}\left[\begin{array}{cc}0 & 1 \\ -1 & 0\end{array}\right]=\operatorname{Pf}\left[\begin{array}{cc}A & D \\ -D^{\mathrm{T}} & B\end{array}\right]$ so we may work with $E$. Now permuting the second and third rows and columns of $E$ does not change the value of the Pfaffian, and leaves us with

$$
E^{\prime}=\left[\begin{array}{cccc}
A & U & D & U \\
-U^{\top} & 0 & V^{\top} & 1 \\
-D^{\top} & -V & B & -V \\
-U^{\top} & -1 & V^{\top} & 0
\end{array}\right] .
$$

Noting that $\left[\begin{array}{cc}D & U \\ -V^{\top} & 1\end{array}\right]$ is rank one we see that we are in the first case ( $k$ even) and the result follows.

Lemma B.2. Consider three skew-symmetric kernels $A, B, C$ defined on some measurable space $(X, \mu)$. Then, assuming that all integrals converge, we have

$$
\begin{aligned}
\frac{1}{n !} \int_{X^{2 n}} \mu^{\otimes 2 n}(d \vec{x}) \operatorname{Pf}\left[(A+B)\left(x_{a}, x_{b}\right)\right]_{a, b=1}^{2 n} & \operatorname{Pf}\left[C\left(x_{a}, x_{b}\right)\right]_{a, b=1}^{2 n} \\
= & \sum_{k_{1}, k_{2} \geq 0, k_{1}+k_{2}=n} \frac{1}{\left(2 k_{1}\right) !\left(2 k_{2}\right) !} \int_{X^{2 n}} \mu^{\otimes 2 n}(d \vec{x}) \operatorname{Pf}\left[A\left(x_{a}, x_{b}\right)\right]_{a, b=1}^{2 k_{1}} \\
& \times \operatorname{Pf}\left[B\left(x_{a}, x_{b}\right)\right]_{a, b=2 k_{1}+1}^{2 k_{1}+2 k_{2}} \operatorname{Pf}\left[C\left(x_{a}, x_{b}\right)\right]_{a, b=1}^{2 n} .
\end{aligned}
$$

Proof. Using several times the definition of the Pfaffian, the fact that the Pfaffian is skewsymmetric, and the symmetry of the integration measure, the left hand side of the identity equals

$$
\begin{aligned}
& \frac{1}{2^{n}(n !)^{2}} \int_{X^{2 n}} \mu^{\otimes 2 n}(d \vec{x}) \sum_{\sigma \in S_{2 n}} \prod_{a=1}^{n}(A+B)\left(x_{\sigma(2 a-1)}, x_{\sigma(2 a)}\right) \operatorname{Pf}\left[C\left(x_{\sigma(a)}, x_{\sigma(b)}\right)\right]_{a, b=1}^{2 n} \\
& =\frac{1}{2^{n} n !} \int_{X^{2 n}} \mu^{\otimes 2 n}(d \vec{x}) \prod_{a=1}^{n}(A+B)\left(x_{2 a-1}, x_{2 a}\right) \operatorname{Pf}\left[C\left(x_{a}, x_{b}\right)\right]_{a, b=1}^{2 n} \\
& =\frac{1}{2^{n} n !} \int_{X^{2 n}} \mu^{\otimes 2 n}(d \vec{x}) \sum_{I \subseteq\{1, \ldots, n\}} \prod_{a \in I} A\left(x_{2 a-1}, x_{2 a}\right) \prod_{a \notin I} B\left(x_{2 a-1}, x_{2 a}\right) \operatorname{Pf}\left[C\left(x_{a}, x_{b}\right)\right]_{a, b=1}^{2 n} \\
& =\frac{1}{2^{n} n !} \sum_{m=0}^{n}\left(\begin{array}{c}
n \\
m
\end{array}\right) \int_{X^{2 n}} \mu^{\otimes 2 n}(d \vec{x}) \prod_{a=1}^{m} A\left(x_{2 a-1}, x_{2 a}\right) \prod_{a=m+1}^{n} B\left(x_{2 a-1}, x_{2 a}\right) \operatorname{Pf}\left[C\left(x_{a}, x_{b}\right)\right]_{a, b=1}^{2 n}
\end{aligned}
$$


where we have used the fact that, for $|I|=m$, the permutation which maps the ordered $m$-tuple formed by the elements in the set $\bigcup_{a \in I}\{2 a-1\} \cup\{2 a\}$ into the $m$-tuple $(1, \ldots, 2 m)$ is even, which implies that the Pfaffian of the kernel $C$ does not change after reordering the variables. Using again the symmetry of the integration measure and the antisymmetry of the Pfaffian we get that the above

$$
\begin{gathered}
=\sum_{m=0}^{n} \frac{1}{2^{n} m !(n-m) !} \int_{X^{2 n}} \mu^{\otimes 2 n}(d \vec{x}) \frac{1}{(2 m) !} \sum_{\sigma_{1} \in S_{2 m}} \prod_{a=1}^{m} A\left(x_{\sigma_{1}(2 a-1)}, x_{\sigma_{1}(2 a)}\right) \\
\times \frac{1}{(2 n-2 m) !} \sum_{\sigma_{2} \in S_{2(n-m)}} \prod_{a=m+1}^{n} B\left(x_{\sigma_{2}(2 a-1)}, x_{\sigma_{2}(2 a)}\right) \operatorname{sgn}\left(\sigma_{1}\right) \operatorname{sgn}\left(\sigma_{2}\right) \operatorname{Pf}\left[C\left(x_{a}, x_{b}\right)\right]_{a, b=1}^{2 n} \\
=\sum_{m=0}^{n} \frac{1}{(2 m) !(2 n-2 m) !} \int_{X^{2 n}} \mu^{\otimes 2 n}(d \vec{x}) \operatorname{Pf}\left[A\left(x_{a}, x_{b}\right)\right]_{a, b=1}^{2 m} \\
\times \operatorname{Pf}\left[B\left(x_{a}, x_{b}\right)\right]_{a, b=2 m+1}^{2 n} \operatorname{Pf}\left[C\left(x_{a}, x_{b}\right)\right]_{a, b=1}^{2 n},
\end{gathered}
$$

which gives the desired result.

Our second integration formula can be regarded as a certain Pfaffian version of the Andréief identity [And83] (sometimes referred to as the generalized Cauchy-Binet identity):

Lemma B.3. Let $(X, \mu)$ be some measurable space and suppose that for every $\vec{x} \in X^{k}$ the matrix $\left[A_{a, b}\left(x_{a}, x_{b}\right)\right]_{a, b=1}^{k}$ is skew-symmetric. Let $B$ be another $k \times k$ skew-symmetric matrix and for $\vec{x} \in X^{k}$ consider another matrix $\left[U_{a, b}\left(x_{b}\right)\right]_{a, b=1}^{k}$. Finally consider functions $\phi_{a}$ defined on $X$. Then

$$
\begin{gathered}
\int_{X^{k}} \mu^{\otimes k}(d \vec{x}) \prod_{a=1}^{k} \phi_{a}\left(x_{a}\right) \operatorname{Pf}\left[\begin{array}{cc}
{\left[A_{a, b}\left(x_{a}, x_{b}\right)\right]_{a, b=1}^{k}} & {\left[U_{a, b}\left(x_{a}\right)\right]_{a, b=1}^{k}} \\
{\left[-U_{b, a}\left(x_{b}\right)\right]_{a, b=1}^{k}} & {\left[B_{a, b}\right]_{a, b=1}^{k}}
\end{array}\right] \\
\quad=\operatorname{Pf}\left[\begin{array}{cc}
{\left[\int_{X^{2}} \mu(d x) \mu\left(d x^{\prime}\right) \phi_{a}(x) \phi_{b}\left(x^{\prime}\right) A_{a, b}\left(x, x^{\prime}\right)\right]_{a, b=1}^{k}} & {\left[\int_{X} \mu(d x) \phi_{a}(x) U_{a, b}(x)\right]_{a, b=1}^{k}} \\
-\left[\int_{X} \mu(d x) \phi_{b}(x) U_{b, a}(x)\right]_{a, b=1}^{k} & {\left[B_{a, b}\right]_{a, b=1}^{k}}
\end{array}\right]
\end{gathered}
$$

provided that all integrals converge.

Proof. Use (B.4) on the left hand side of the claimed identity and expand the Pfaffian using its definition to see that it equals

$$
\begin{aligned}
& \frac{1}{2^{k} k !} \sum_{\sigma \in S_{2 k}} \operatorname{sgn}(\sigma) \int_{X^{k}} \mu\left(d x_{1}\right) \cdots \mu\left(d x_{k}\right) \\
& \times \prod_{\substack{\sigma(2 a-1) \leq k \\
\sigma(2 a) \leq k}} A_{\sigma(2 a-1), \sigma(2 a)}\left(x_{\sigma(2 a-1)}, x_{\sigma(2 a)}\right) \phi_{\sigma(2 a-1)}\left(x_{\sigma(2 a-1)}\right) \phi_{\sigma(2 a)}\left(x_{\sigma(2 a)}\right)
\end{aligned}
$$

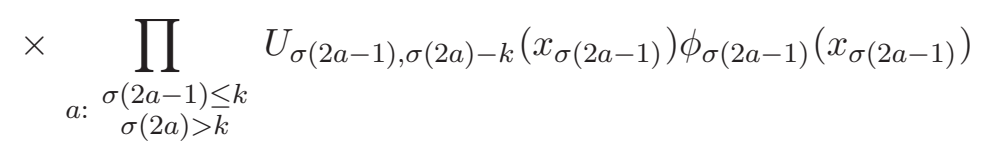

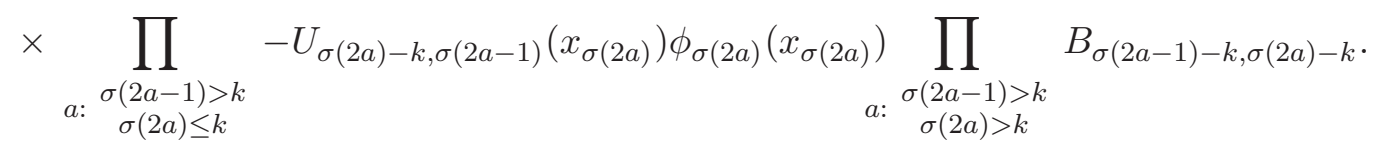

Since each $x_{a}(a=1, \ldots, k)$ appears in one and only one of the above factors, the integrals can be brought inside the corresponding factor, and now forming the resulting Pfaffian gives the identity. 
B.2. Fredholm determinants. It is instructive to review briefly the theory of Fredholm determinants on $L^{2}$ spaces before discussing Fredholm Pfaffians. For more details see Section 2 of [QR14].

Let $A$ by an $m \times m$ matrix, write $[m]=\{1, \ldots, m\}$ and let $\left(\begin{array}{c}{[m]} \\ \ell\end{array}\right)$ denote the family of subsets of $[m]$ of size $\ell$. A standard calculation shows that, for $\lambda \in \mathbb{C}$,

$$
\operatorname{det}(I+\lambda A)=\sum_{\ell=0}^{m} \lambda^{\ell} \sum_{S \in\left(\begin{array}{c}
{[m]} \\
\ell
\end{array}\right)} \operatorname{det}\left(A_{S \times S}\right)
$$

with self-explanatory notation. This suggests a way of extending the determinant to the infinite dimensional case. In the case of $L^{2}$ spaces it leads to the following.

Let $(X, \Sigma, \mu)$ be a measure space and $K: X \times X \longrightarrow \mathbb{R}$ be a kernel which defines an integral operator acting on $L^{2}(X)$ through $K f(x)=\int_{X} \mu(d y) K(x, y) f(y)$. The Fredholm determinant of $K$ (on $\left.L^{2}(X)\right)$ is defined to be the (formal) power series

$$
\operatorname{det}[I+\lambda K]_{L^{2}(X)}=\sum_{k=0}^{\infty} \frac{\lambda^{k}}{k !} \int_{X^{k}} \mu\left(d x_{1}\right) \ldots \mu\left(d x_{n}\right) \operatorname{det}\left[K\left(x_{a}, x_{b}\right)\right]_{a, b=1}^{k} .
$$

We will omit the subscript $L^{2}(X)$ in the determinant when no confusion can arise. This identity can be regarded as a numerical identity whenever the right hand side is absolutely convergent. This is the case, for instance, whenever $|K(x, y)| \leq C$ for all $x, y \in X$ and some $C>0$, thanks to Hadamard's bound.

An interesting class of operators for which the Fredholm determinant power series is absolutely convergent is the family of trace class operators. We recall that an operator $K: L^{2}(X) \longrightarrow L^{2}(X)$ is said to be trace class if it has finite trace norm:

$$
\|K\|_{1}:=\sum_{n \geq 1} \int_{X} \mu(d x) \psi_{n}(x)|K| \psi_{n}(x)<\infty
$$

where $\left(\psi_{n}\right)_{n \geq 0}$ is any orthonormal basis of $L^{2}(X)$ and $|K|=\sqrt{K^{*} K}$ is the unique positive square root of the operator $K^{*} K$. Such an operator is necessarily compact, and in this case one has

$$
\operatorname{det}[I+z K]=\prod_{k \geq 1}\left(1+z \lambda_{k}\right)
$$

where the $\lambda_{k}$ 's are the eigenvalues of $K$. This identity (known as Lidskii's Theorem) is highly non-trivial, and provides one possible way to extend the definition of the Fredholm determinant to trace class operators on a general separable Hilbert space. A nice property of the Fredholm determinant restricted to trace class operators is that it is continuous: for $K_{1}$ and $K_{2}$ trace class one has

$$
\left|\operatorname{det}\left[I+K_{1}\right]-\operatorname{det}\left[I+K_{2}\right]\right| \leq\left\|K_{1}-K_{2}\right\|_{1} e^{\left\|K_{1}\right\|_{1}+\left\|K_{2}\right\|_{1}+1} .
$$

This inequality follows from a rather general (and simple) argument based on the analiticity in $\lambda \in \mathbb{C}$ of the function $\operatorname{det}[I+\lambda K]$ and the inequality $|\operatorname{det}[I+\lambda K]| \leq e^{|\lambda|\|K\|_{1}}$ (which essentially follows from (B.8)), see Theorem 3.4 of [Sim05] or Corollary II.4.2 of [GGK00] (see also the discussion following (2.9) in [QR14]).

Another useful fact about Fredholm determinants is the so-called cyclic property: if $K_{1}: L^{2}\left(X_{1}\right) \longrightarrow L^{2}\left(X_{2}\right)$ and $K_{1}: L^{2}\left(X_{2}\right) \longrightarrow L^{2}\left(X_{1}\right)$ then

$$
\operatorname{det}\left[I+K_{1} K_{2}\right]=\operatorname{det}\left[I+K_{2} K_{1}\right]
$$

whenever the two sides are absolutely convergent (e.g. if both $K_{1} K_{2}$ and $K_{2} K_{1}$ are trace class). An important consequence of this fact is the ability to conjugate an operator without changing its Fredholm determinant:

$$
\operatorname{det}\left[I+V^{-1} K V\right]=\operatorname{det}[I+K]
$$


whenever both sides make sense. This is often used to replace $K$ by a conjugate trace class kernel. For more on all this and on extensions to other separable Hilbert spaces see [Sim05; GGK00].

Finally let us briefly recall the notion of regularized determinants, since a similar notion will be introduced below in the Pfaffian case. Suppose that $K$ is a Hilbert-Schmidt operator (see [QR14] for the definition) and recall that the product of two Hilbert-Schmidt operators is trace class. We define the regularized determinant of $K$ as

$$
\operatorname{det}_{2}[I+K]=\operatorname{det}\left[(I+K) e^{-K}\right] .
$$

Note that $(I+K) e^{-K}-I=\sum_{n \geq 2} \frac{(-1)^{n+1}(n-1)}{n !} K^{n}$ is a trace class operator, so the definition makes sense for any Hilbert-Schmidt operator $K$ (one can also define regularized determinants of higher order, but we will not do it here). It can be shown that the analog of (B.8) for the regularized determinant is

$$
\operatorname{det}_{2}[I+K]=\prod_{k \geq 1}\left(1+\lambda_{k}\right) e^{-\lambda_{k}}
$$

which further implies that $\left|\operatorname{det}_{2}[I+K]\right| \leq e^{\frac{1}{2}\|K\|_{2}^{2}}$. Using this bound one can obtain the analog of (B.9) (by the same argument): if $K_{1}$ and $K_{2}$ are Hilbert-Schmidt operators then

$$
\left|\operatorname{det}_{2}\left[I+K_{1}\right]-\operatorname{det}_{2}\left[I+K_{2}\right]\right| \leq\left\|K_{1}-K_{2}\right\|_{2} e^{\frac{1}{2}\left(\left\|K_{1}\right\|_{2}+\left\|K_{1}\right\|_{2}+1\right)^{2}},
$$

which in particular gives continuity of the regularized determinant with respect to the Hilbert-Schmidt norm. Another way in which this notion can be useful is the following. Suppose one knew that both $\operatorname{det}[I+K]$ and $\operatorname{det}\left[e^{-K}\right]$ are given by asolutely convergent series. Then $\operatorname{det}\left[(I+K) e^{-K}\right]=\operatorname{det}[I+K] \operatorname{det}\left[e^{-K}\right]$ (which follows from the general property $\left.\operatorname{det}\left[\left(I+K_{1}\right)\left(I+K_{2}\right)\right]=\operatorname{det}\left[I+K_{1}\right] \operatorname{det}\left[I+K_{2}\right]\right)$. The left hand side can be controled in terms of the Hilbert-Schmidt norm of $K$, so if one has some additional control on $\operatorname{det}\left[e^{-K}\right]$ (observe, in particular, that if $K$ is nice enough, e.g. trace class, then $\operatorname{det}\left[e^{-K}\right]=e^{-\operatorname{tr}[K]}$ ) this can be used to control $\operatorname{det}[I+K]$. For much more on this see [Sim05; GGK00].

B.3. Fredholm Pfaffians on $L^{2}$ spaces. We turn now to Fredholm Pfaffians. Given $n \in \mathbb{Z}_{\geq 1}$ we define $J$ to be the $2 n \times 2 n$ block-diagonal matrix which has $2 \times 2$ blocks on the diagonal, all equal to $\left[\begin{array}{cc}0 & 1 \\ -1 & 0\end{array}\right]$. If $A$ is a $2 n \times 2 n$ skew-symmetric matrix then one can show that

$$
\operatorname{Pf}(J+\lambda A)=\sum_{\ell=0}^{m} \lambda^{\ell} \sum_{S \in\left(\begin{array}{c}
{[m]} \\
\ell
\end{array}\right)} \operatorname{Pf}\left(A_{S \times S}\right) .
$$

This is, of course, the Pfaffian analogue of (B.7), and suggests the following infinite dimensional extension [Rai00] (note that the above sum actually involves only even $\ell$, since $\operatorname{Pf}\left(A_{S \times S}\right)=0$ otherwise $)$

Consider a skew-symmetric $2 \times 2$-matrix kernel

$$
K\left(\lambda_{1}, \lambda_{2}\right)=\left[\begin{array}{cc}
K_{1,1}\left(\lambda_{1}, \lambda_{2}\right) & K_{1,2}\left(\lambda_{1}, \lambda_{2}\right) \\
-K_{1,2}\left(\lambda_{2}, \lambda_{1}\right) & K_{2,2}\left(\lambda_{1}, \lambda_{2}\right)
\end{array}\right]
$$

(the skew-symmetry condition in this case translates into $K_{a, a}\left(\lambda_{1}, \lambda_{2}\right)=-K_{a, a}\left(\lambda_{2}, \lambda_{1}\right)$ for $a=1,2)$. We regard $K$ as an integral operator acting on $f \in L^{2}(X) \oplus L^{2}(X)$ as follows:

$$
(K f)_{b}(x)=\sum_{a=1}^{2} \int_{X} \mu(d y) K_{b, a}(x, y) f_{a}(y) \quad \text { for } b=1,2 .
$$

For any such kernel we define its Fredholm Pfaffian on $L^{2}(X)$ as the (formal) power series

$$
\operatorname{Pf}[J+\lambda K]_{L^{2}(X)}=\sum_{k \geq 0} \frac{\lambda^{k}}{k !} \int_{X^{k}} \mu\left(d x_{1}\right) \cdots \mu\left(d x_{k}\right) \operatorname{Pf}\left[K\left(x_{a}, x_{b}\right)\right]_{a, b=1}^{k}
$$


whenever the right hand side is convergent. As for Fredholm determinants, we will usually omit the subscript $L^{2}(X)$ in the Pfaffian ${ }^{6}$.

Observe that for a $2 n \times 2 n$ skew-symmetric matrix $A$ we have by (B.3) that

$$
\operatorname{Pf}(J+A)^{2}=\operatorname{Pf}\left(J\left(I+J^{-1} A\right)\right)^{2}=\operatorname{det}(J) \operatorname{det}\left(I+J^{-1} A\right)=\operatorname{det}(I-J A),
$$

where we have used the facts that $\operatorname{det}(J)=1$ and $J^{2}=-I$. The following result extends this to a relationship between Fredholm determinants on $L^{2}(X) \oplus L^{2}(X)$ and Fredholm Pfaffians on $L^{2}(X)$.

Proposition B.4. For any skew-symmetric $2 \times 2$-matrix kernel $K$ and any $\lambda \in \mathbb{C}$, as long as the integrals in the series (B.7) and (B.12) are convergent, we have

$$
\operatorname{Pf}[J+\lambda K]_{L^{2}(X)}^{2}=\operatorname{det}[I-\lambda J K]_{L^{2}(X) \oplus L^{2}(X)},
$$

where the identity is in the sense of formal power series.

Proof. Let $\operatorname{Pf}[J+\lambda K]=\sum \lambda^{n} b_{n}$ and $\operatorname{det}[I-\lambda J K]=\sum \lambda^{n} a_{n}$. If $K$ is finite-rank then (B.13) implies that $a_{n}=\sum_{j=0}^{n}\left(\begin{array}{c}n \\ j\end{array}\right) b_{j} b_{n-j}$. In the general case the kernel $K$ can be approximated by discretization (as explained in Section 2 of [QR14]) to see that the relation between the coefficients of the power series still holds, which proves the identity.

Of course, (B.14) is to be regarded as a numerical identity whenever both sides are absolutely convergent. As we will see next, this is the case when $K$ is trace class.

Proposition B.5. If $K$ is a skew-symmetric $2 \times 2$-matrix kernel which defines a trace class operator on $L^{2}(X) \oplus L^{2}(X)$, then both sides of identity (B.14) define absolutely convergent series, and in particular the identity is numerical. Moreover, if the Fredholm Pfaffian series of $K$ is given by $\operatorname{Pf}[J+\lambda K]=\sum_{n \geq 0} a_{n} \lambda^{n}$, then $\sum_{n \geq 0}\left|a_{n} \lambda^{n}\right| \leq e^{\frac{1}{2}|\lambda|\|K\|_{1}}$.

Proof. The fact that the right hand side of the identity defines an absolutely convergent series follows from our discussion about Fredholm determinants and the fact that $|J K|=$ $|K|$, so that $\|J K\|_{1}=\|K\|_{1}$. Now suppose that $A$ is a $2 n \times 2 n$ skew-symmetric matrix and let $z \in \mathbb{C}$. Since $J+z A$ has pure imaginary eigenvalues in complex conjugate pairs and $\operatorname{det}(J+z A)=-\operatorname{det}(I-z J A)$, we know that the eigenvalues of $J A$ are real and they all have even multiplicity. This means that $\operatorname{det}(I-z J A)=\operatorname{Pf}(J+z A)^{2}=\prod_{a}\left(1-z \lambda_{j}\right)^{2}$ where the $\lambda_{j}$ 's are the eigenvalues of $J A$ (appearing with half their multiplicity). This identity can be extended by approximation to $K$ since $K$ is trace class, and this determines the Fredholm Pfaffian series for $K$ up to sign:

$$
\operatorname{Pf}[J+z K]=\sum_{n \geq 0} a_{n} z^{n}=\sigma \prod_{j}\left(1-z \lambda_{j}\right)
$$

for $\sigma \in\{-1,1\}$. In particular, this means that the $n$-th term of the Fredholm Pfaffian series is given by $a_{n}=\sigma z^{n} \sum_{j_{1}<\cdots<j_{n}} \lambda_{j_{1}} \cdots \lambda_{j_{n}}$. Now $\left|\sum_{j_{1}<\cdots j_{n}} \lambda_{j_{1}} \cdots \lambda_{j_{n}}\right| \leq \frac{1}{n !}\left(\frac{1}{2}\|J K\|\right)_{1}^{n}$ by Lemma 3.3 and (3.8) in [Sim05] (the $1 / 2$ is because each eigenvalue is counted with half its multiplicity). Since $\|J K\|_{1}=\|K\|_{1}$ as explained above, we have proved that

$$
\sum_{n \geq 0}\left|a_{n} z^{n}\right| \leq \sum_{n \geq 0} \frac{1}{n !}|z|^{n}\left(\frac{1}{2}\|K\|_{1}\right)^{n}=e^{\frac{1}{2}|z|\|K\|_{1}} .
$$

An important consequence of the last result is that the Fredholm Pfaffian restricted to trace class operators is continuous: if $K_{1}$ and $K_{2}$ are trace class skew-symmetric $2 \times 2$ matrix kernels then

$$
\left|\operatorname{Pf}\left[J+K_{1}\right]-\operatorname{Pf}\left[J+K_{2}\right]\right| \leq\left\|K_{1}-K_{2}\right\|_{1} e^{\frac{1}{2}\left(\left\|K_{1}\right\|_{1}+\left\|K_{2}\right\|_{1}+1\right)} .
$$

\footnotetext{
${ }^{6}$ Observe that, although $K$ acts on $L^{2}(X) \oplus L^{2}(X)$, we are declaring this to be the Fredholm Pfaffian on $L^{2}(X)$. This is just a matter of convention, so we make this choice for notational convenience.
} 
Like (B.11), this inequality can be proved in the same way as (B.9), based now on the inequality $|\operatorname{Pf}[J+K]| \leq e^{\frac{1}{2}\|K\|_{1}}$ implicit in Proposition B.5.

We mention also two Fredholm Pfaffian analogs of (B.10). The first one follows from (B.3) by approximation and says that

$$
\operatorname{Pf}\left[\left(I+L^{\top}\right)(I+K)(I+L)\right]=\operatorname{det}[I+L] \operatorname{Pf}[I+K]
$$

as long as both sides define absolutely convergent power series. For the second one, let $M$ be a symplectic $2 \times 2$ matrix kernel. Then, as long as both sides define absolutely convergent power series, we have

$$
\operatorname{Pf}[J+\lambda K]=\operatorname{Pf}\left[J+\lambda M^{\top} K M\right] .
$$

The proof of (B.17) is elementary:

$$
\begin{aligned}
& \operatorname{Pf}\left[J+\lambda M^{\top} K M\right]=\sum_{k \geq 0} \frac{\lambda^{k}}{k !} \int_{X^{k}} \mu\left(d x_{1}\right) \cdots \mu\left(d x_{k}\right) \operatorname{Pf}\left[\left(M^{\top} K M\right)\left(x_{a}, x_{b}\right)\right]_{a, b=1}^{k} \\
& \quad=\sum_{k \geq 0} \frac{\lambda^{k}}{k !} \int_{X^{k}} \mu\left(d x_{1}\right) \cdots \mu\left(d x_{k}\right) \operatorname{det}\left[M\left(x_{a}, x_{b}\right)\right]_{a, b=1}^{k} \operatorname{Pf}\left[K\left(x_{a}, x_{b}\right)\right]_{a, b=1}^{k}=\operatorname{Pf}[J-K],
\end{aligned}
$$

where we have used (B.3) and the fact that the determinant of any symplectic matrix equals 1.

One can in principle use (B.17) to replace $K$ by a trace class kernel and then use the (better-developed, and in a sense simpler) theory of Fredholm determinants to study the Fredholm Pfaffian of $K$. On the other hand, even if this is not possible one can still use Proposition B.4 to reduce the study of a Fredholm Pfaffian to a Fredholm determinant, as long as they are both convergent. This is in fact the situation we are in in Section A.3, where we are dealing with a kernel $K$ for which there is no obvious symplectic $2 \times 2$-matrix kernel such that $M^{\top} K M$ is trace class, but the reduction to a Fredholm determinant is still very useful.

Let us finish by introducing a notion of regularized Fredholm Pfaffians. Suppose that $K$ is a skew-symmetric $2 \times 2$-matrix kernel which defines a Hilbert-Schmidt operator. Observe that $J$ is symplectic, and thus by (B.17) we have

$$
\operatorname{Pf}[J+K]=\operatorname{Pf}\left[J+J^{\top} K J\right]=\operatorname{Pf}[J-J K J] .
$$

Observe also that $(J K)^{\top}=K J$. In view of these two facts we define the regularized Pfaffian of $K$ as

$$
\operatorname{Pf}_{2}[J+K]=\operatorname{Pf}\left[(J-J K J) e^{K J}\right]=\operatorname{Pf}\left[e^{\frac{1}{2} J K}(J-J K J) e^{\frac{1}{2} K J}\right] .
$$

The second equality actually holds at the level of the operators inside the two Fredholm Pfaffians (as can be checked easily using the series expansion of the exponential) and has the advantage of making the skew-symmetry of the argument more apparent. A calculation shows that $(J-J K J) e^{K J}-J=-\sum_{n \geq 2} \frac{n-1}{n !}(J K)^{n} J$, and thus the right hand side of (B.18) makes sense as the Fredholm Pfaffian of a trace class operator (since $J K$ is Hilbert-Schmidt, and thus $(J K)^{n}$ is trace class for any $\left.n \geq 2\right)$. Note also that

$$
\operatorname{Pf}_{2}[J+K]^{2}=\operatorname{det}_{2}[I-J K] .
$$

Additionally, if $K_{1}$ and $K_{2}$ are Hilbert-Schmidt then

$$
\left|\mathrm{Pf}_{2}\left[J+K_{1}\right]-\operatorname{Pf}_{2}\left[J+K_{2}\right]\right| \leq\left\|K_{1}-K_{2}\right\|_{2} e^{\frac{1}{4}\left(\left\|K_{1}\right\|_{2}+\left\|K_{1}\right\|_{2}+1\right)^{2}}
$$

(with the same proof as (B.9), now based on the inequality $\left|\operatorname{Pf}_{2}[J+K]\right|=\sqrt{\left|\operatorname{det}_{2}[I-J K]\right|} \leq$ $\left.e^{\frac{1}{4}\|K\|_{2}^{2}}\right)$.

Observe that if one knew in addition that $K$ is trace class, then by (B.16) one would have

$$
\operatorname{Pf}_{2}[J+K]=\operatorname{det}\left[e^{\frac{1}{2} J K}\right] \operatorname{Pf}[J-J K J]=e^{\frac{1}{2} \operatorname{tr}[J K]} \operatorname{Pf}[J+K] .
$$


As in the case of the regularized determinants discussed in Section B.2, one situation in which the introduction of regularized Pfaffians can be useful is when $K$ is Hilbert-Schmidt but not trace class, but on the other hand some additional control on $K$ is available which makes it possible to show that the first or both equalities in (B.20) hold. In such a case, since the left hand side can be controlled by the Hilbert-Schmidt norm of $K$ (which is the same as that of $J K$ ), this identity provides a possible route for controlling $\operatorname{Pf}[J+K]$. This idea was used at the end of the proof of Proposition A.4 to use continuity in $r$ of a certain kernel $K_{r}$ with respect to the Hilbert-Schmidt norm, in order to upgrade the identity $\operatorname{Pf}\left[J-K_{r}\right]^{2}=\operatorname{det}\left[I+J K_{r}\right]$ to $\operatorname{Pf}\left[J-K_{r}\right]=\sqrt{\operatorname{det}\left[I+J K_{r}\right]}$.

Acknowledgements. The authors would like to thank Alexei Borodin, Ivan Corwin, Pierre Le Doussal, and Pasquale Calabrese for numerous discussions about the results in this paper. JO was partially supported by the Natural Sciences and Engineering Research Council of Canada. JQ gratefully acknowledges financial support from the Natural Sciences and Engineering Research Council of Canada, the I. W. Killam Foundation, and the Institute for Advanced Study. DR was partially supported by Fondecyt Grant 1120309, by Conicyt Basal-CMM, and by Programa Iniciativa Científica Milenio grant number NC130062 through Nucleus Millenium Stochastic Models of Complex and Disordered Systems.

\section{REFERENCES}

[ACQ11] G. Amir, I. Corwin, and J. Quastel. Probability distribution of the free energy of the continuum directed random polymer in $1+1$ dimensions. Comm. Pure Appl. Math. 64.4 (2011), pp. 466-537.

[And83] C. Andréief. Note sur une relation entre les intégrales définies des produits des fonctions. Mém. de la Soc. Sci. Bordeaux 2 (1883), pp. 1-14.

[BC14] A. Borodin and I. Corwin. Macdonald processes. Probab. Theory Related Fields 158.1-2 (2014), pp. 225-400.

[BCF12] A. Borodin, I. Corwin, and P. Ferrari. Free energy fluctuations for directed polymers in random media in $1+1$ dimension. To appear in Comm. Pure. Appl. Math. 2012. arXiv:1204.1024.

[BCFV14] A. Borodin, I. Corwin, P. Ferrari, and B. Vetö. Height fluctuations for the stationary KPZ equation. 2014. arXiv:1407.6977.

[BCS14] A. Borodin, I. Corwin, and T. Sasamoto. From duality to determinants for q-TASEP and ASEP. Ann. Probab. 42.6 (Nov. 2014), pp. 2314-2382.

[CD14] P. Calabrese and P. L. Doussal. Interaction quench in a Lieb-Liniger model and the KPZ equation with flat initial conditions. J. Stat. Mech. Theor. Exp. 2014.5 (2014), P05004.

[CDR10] P. Calabrese, P. L. Doussal, and A. Rosso. Free-energy distribution of the directed polymer at high temperature. EPL (Europhysics Letters) 90.2 (2010), p. 20002.

[CQ13] I. Corwin and J. Quastel. Crossover distributions at the edge of the rarefaction fan. Ann. Probab. 41 (2013), pp. 1243-1314.

[DeB55] N. G. DeBruijn. On some multiple integrals involving determinants. J. Indian Math. Soc. (N.S.) 19 (1955), pp. 133-151.

[DG09] P. Deift and D. Gioev. Random matrix theory: invariant ensembles and universality. Vol. 18. Courant Lecture Notes in Mathematics. Courant Institute of Mathematical Sciences (New York); American Mathematical Society (Providence, RI), 2009, pp. $\mathrm{x}+217$.

[DNWBC14] J. De Nardis, B. Wouters, M. Brockmann, and J.-S. Caux. Solution for an interaction quench in the Lieb-Liniger Bose gas. Phys. Rev. A 89 (3 2014), p. 033601. 
[Dot10] V. Dotsenko. Bethe ansatz derivation of the Tracy-Widom distribution for one-dimensional directed polymers. EPL (Europhysics Letters) 90.2 (2010), p. 20003.

[Fer04] P. L. Ferrari. Polynuclear growth on a flat substrate and edge scaling of GOE eigenvalues. Comm. Math. Phys. 252.1-3 (2004), pp. 77-109.

[FS05] P. L. Ferrari and H. Spohn. A determinantal formula for the GOE TracyWidom distribution. J. Phys. A 38.33 (2005), pp. L557-L561.

[FT95] I. B. Frenkel and V. G. Turaev. Trigonometric solutions of the Yang-Baxter equation, nets, and hypergeometric functions. In: Functional analysis on the eve of the 21st century, Vol. 1 (New Brunswick, NJ, 1993). Vol. 131. Progr. Math. Boston, MA: Birkhäuser Boston, 1995, pp. 65-118.

[FV13] P. Ferrari and B. Vető. Tracy-Widom asymptotics for q-TASEP. 2013. arXiv:1310.2515.

[GGK00] I. Gohberg, S. Goldberg, and N. Krupnik. Traces and determinants of linear operators. Vol. 116. Operator Theory: Advances and Applications. Basel: Birkhäuser Verlag, 2000, pp. $\mathrm{x}+258$.

[GR04] G. Gasper and M. Rahman. Basic hypergeometric series. Second. Vol. 96. Encyclopedia of Mathematics and its Applications. With a foreword by Richard Askey. Cambridge: Cambridge University Press, 2004, pp. xxvi+428.

[Hai13] M. Hairer. Solving the KPZ equation. Ann. of Math. (2) 178.2 (2013), pp. 559664.

[Hai14] M. Hairer. A theory of regularity structures. Invent. Math. 198.2 (2014), pp. 269-504.

[IS11] T. Imamura and T. Sasamoto. Replica approach to the KPZ equation with the half Brownian motion initial condition. J. Phys. A 44.38 (2011), pp. 385001, 29.

[IS13] T. Imamura and T. Sasamoto. Stationary correlations for the 1D KPZ equation. J. Stat. Phys. 150.5 (2013), pp. 908-939.

[LDC12] P. Le Doussal and P. Calabrese. The KPZ equation with flat initial condition and the directed polymer with one free end. J. Stat. Mech. 2012.06 (2012), P06001.

[Lee10] E. Lee. Distribution of a particle's position in the ASEP with the alternating initial condition. J. Stat. Phys. 140.4 (2010), pp. 635-647.

[MFQR15] G. Moreno Flores, J. Quastel, and D. Remenik. Intermediate disorder limits for directed polymers with boundary conditions. In preparation. 2015.

[NG94] C. A. Nelson and M. G. Gartley. On the zeros of the $q$-analogue exponential function. J. Phys. A 27.11 (1994), pp. 3857-3881.

[OQR14] J. Ortmann, J. Quastel, and D. Remenik. Exact formulas for random growth with half-flat initial data. 2014. arXiv:1407.8484.

[QR14] J. Quastel and D. Remenik. Airy processes and variational problems. In: Topics in Percolative and Disordered Systems. Ed. by A. Ramírez, G. Ben Arous, P. A. Ferrari, C. Newman, V. Sidoravicius, and M. E. Vares. Vol. 69. Springer Proceedings in Mathematics \& Statistics. 2014, pp. 121-171.

[Rai00] E. M. Rains. Correlation functions for symmetrized increasing subsequences. 2000. arXiv:math/0006097.

[Sim05] B. Simon. Trace ideals and their applications. Second. Vol. 120. Mathematical Surveys and Monographs. American Mathematical Society, 2005, pp. viii+150.

[TW08a] C. A. Tracy and H. Widom. A Fredholm determinant representation in ASEP. J. Stat. Phys. 132.2 (2008), pp. 291-300.

[TW08b] C. A. Tracy and H. Widom. Integral formulas for the asymmetric simple exclusion process. Comm. Math. Phys. 279.3 (2008), pp. 815-844. See also erratum: Comm. Math. Phys. 304.3 (2011), pp. 875-878.

[TW09a] C. A. Tracy and H. Widom. Asymptotics in ASEP with step initial condition. Comm. Math. Phys. 290.1 (2009), pp. 129-154. 
[TW09b] C. A. Tracy and H. Widom. On ASEP with step Bernoulli initial condition. J. Stat. Phys. 137.5-6 (2009), pp. 825-838.

[TW96] C. A. Tracy and H. Widom. On orthogonal and symplectic matrix ensembles. Comm. Math. Phys. 177.3 (1996), pp. 727-754.

[VSI97] O. Valle, M. Soares, and C. de Izarra. An integral representation for the product of Airy functions. English. Zeitschrift fr angewandte Mathematik und Physik ZAMP 48.1 (1997), pp. 156-160.

[Wid41] D. V. Widder. The Laplace Transform. Princeton Mathematical Series, v. 6. Princeton, N. J.: Princeton University Press, 1941, pp. x+406.

(J. Ortmann) Department of Mathematics, University of Toronto, 40 St. George Street, Toronto, Ontario, Canada M5S 2E4

E-mail address: janosch.ortmann@utoronto.ca

(J. Quastel) Department of Mathematics, University of Toronto, 40 St. George Street, Toronto, Ontario, Canada M5S 2E4

E-mail address: quastel@math.toronto.edu

(D. Remenik) Departamento de Ingeniería Matemática and Centro de Modelamiento Matemático, Universidad de Chile, Av. Beauchef 851, Torre Norte, Santiago, Chile

E-mail address: dremenik@dim.uchile.cl 